\title{
The Memorization, Preparation, and Performance of Piano Music: Cognitive Foundations and Current Neuro-Music Research
}

Amy M. Simpson

ams0065@mix.wvu.edu

Follow this and additional works at: https://researchrepository.wvu.edu/etd

Part of the Other Music Commons

\section{Recommended Citation}

Simpson, Amy M., "The Memorization, Preparation, and Performance of Piano Music: Cognitive Foundations and Current Neuro-Music Research" (2021). Graduate Theses, Dissertations, and Problem Reports. 8297.

https://researchrepository.wvu.edu/etd/8297

This Dissertation is protected by copyright and/or related rights. It has been brought to you by the The Research Repository @ WVU with permission from the rights-holder(s). You are free to use this Dissertation in any way that is permitted by the copyright and related rights legislation that applies to your use. For other uses you must obtain permission from the rights-holder(s) directly, unless additional rights are indicated by a Creative Commons license in the record and/ or on the work itself. This Dissertation has been accepted for inclusion in WVU Graduate Theses, Dissertations, and Problem Reports collection by an authorized administrator of The Research Repository @ WVU.

For more information, please contact researchrepository@mail.wvu.edu. 
The Memorization, Preparation, and Performance of Piano Music:

Cognitive Foundations and Current Neuro-Music Research

Amy M. Simpson

Research submitted

to the College of Creative Arts

at West Virginia University

in partial fulfillment of the requirements for the degree of

Doctor of Musical Arts in

Piano Performance

Peter Amstutz, DMA, Chair

Robert Chafin, MM

Andrew Kohn, PhD

Lucy Mauro, DMA

Bernard Schultz, PhD

Research Consultant

Sarah E. Allen, PhD, Southern Methodist University

School of Music

Morgantown, West Virginia

2021

Keywords: piano performance, memorization, memory, learning, music practice, auditory imagery, planning, metacognition 


\begin{abstract}
The Memorization, Preparation, and Performance of Piano Music:

Cognitive Foundations and Current Neuro-Music Research
\end{abstract}

Amy M. Simpson

Memorization of piano music for performance has often been shrouded in mystery. A body of neuro-music research has emerged, mostly in the last twenty years, that addresses how musicians perceive, learn, and memorize music, and how these processes operate in music performance. This paper is a compendium of current research on music perception, learning, memorization, and performance, specifically focused on piano music and piano performance. It is intended to benefit both pianists (performers, teachers, students), and neuro-music researchers.

This research explains the operation of human memory systems and, from this platform, addresses aspects of music memorization such as multi-modal approaches and individual differences. A discussion of motor learning precedes current research comparing it with perceptual learning.

Further, this work discusses memory acquisition, stabilization, and sleep consolidation, supported by current piano-specific research. It considers the role of the original modality of learning, and reviews the significance of metacognition and attention. Additionally, this paper presents information on auditory imagery in practice and performance, and on methods for developing musical expressiveness.

A final section summarizes the most significant implications for musicians and important directions for future research. 


\section{Acknowledgements}

I owe a debt of gratitude to my applied teacher and committee chair, Dr. Peter Amstutz, for his continued support of this project. His encouragement in finding the necessary resources and his patience with my extended study of neuro-music helped to enable this work. I am forever grateful for his support and guidance in both my academic and performance pursuits.

I offer special thanks to my doctoral committee, Dr. Robert Chafin, Dr. Andrew Kohn, Dr. Lucy Mauro, and Dr. Bernard Schultz, who, each in their own way, have supported me as a graduate student and as a performer.

This project could not have been possible without Dr. Sarah E. Allen, my research consultant, who provided scholarly and caring advice, and a great deal of encouragement to me as a newcomer to the field of neuro-music. Dr. Allen holds a PhD in Music and Human Learning from the University of Texas at Austin. While teaching at Southern Methodist University, Meadows School of the Arts, she actively performs piano-specific neuro-music research. Her faith in my work and her guidance were invaluable. I will always be in her debt for her willingness to help.

Finally, I give the most special thanks of all to my husband, Jim, for his support and patience during the preparation of this research and for enabling the entire pursuit of my doctoral degree, which has now become a reality. 


\section{TABLE OF CONTENTS}

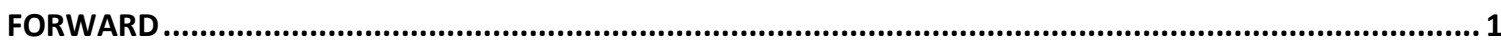

INTRODUCTION. MEMORIZATION IN HISTORY; MEMORIZATION TODAY............................................ 2

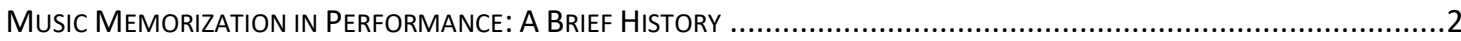

Memorization Today: The Significance of Music Cognition ReSEARCh .............................................

HUMAN MEMORY SYSTEMS AND MUSIC: CONCEPTS AND CURRENT PERSPECTIVES FROM PSYCHOLOGY

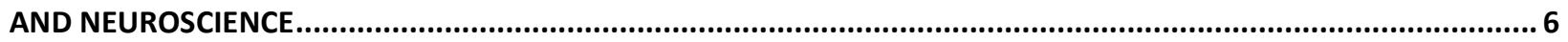

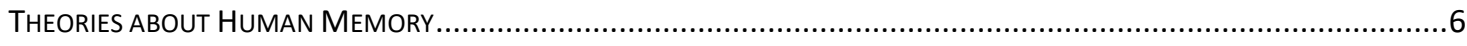

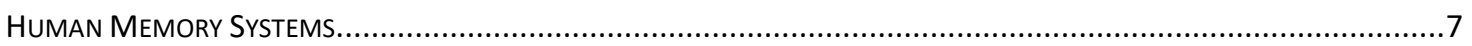

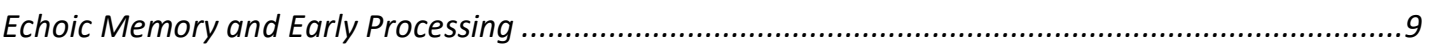

Short-Term Memory and Grouping .................................................................................... 10

Short-Term Memory Capacity and Chunking ............................................................................. 12

Activation of Long-Term Memory and Interchange with Short-Term Memory................................13

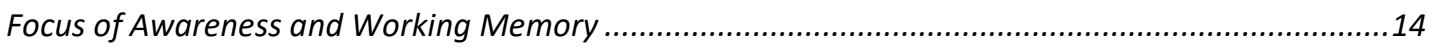

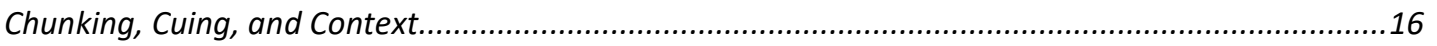

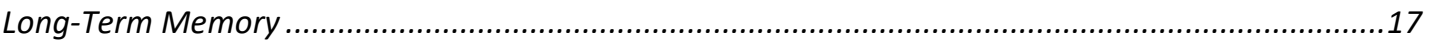

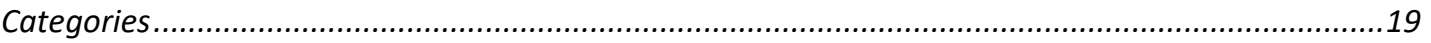

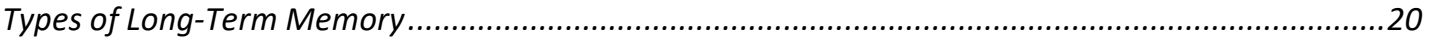

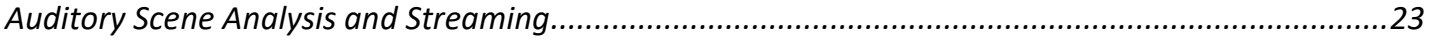

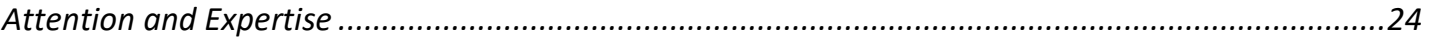

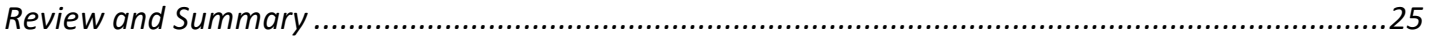

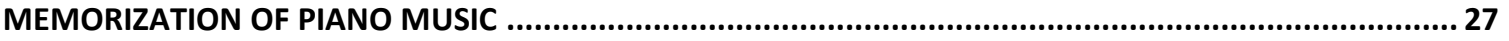

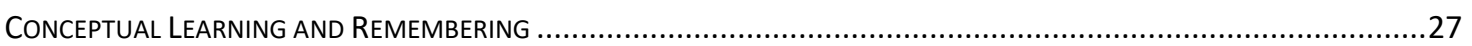

Understanding Structure. Score Analysis, Silent Learning, and Memorization..................................27

Hear First, See First, or Play First? The Role of Modality in the Original Learning Process...................29

Role of Familiarity with a Piece, Familiarity with a Style, and Likeability.........................................32

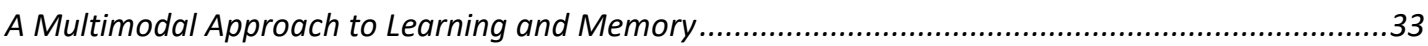

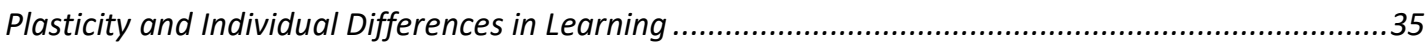

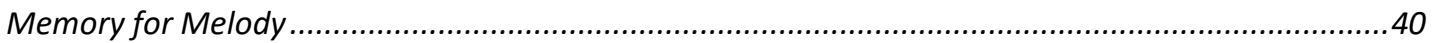

Memorization, Virtuosity, and Expertise in Piano Music .........................................................43

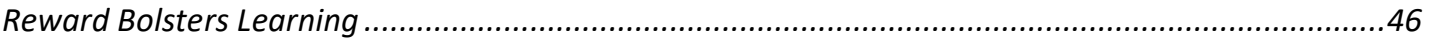


Is Music Memory Special? ....................................................................................................47

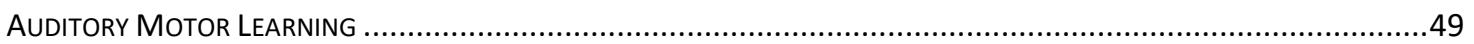

Generalized Motor Programs and Motor Schemas ..................................................................49

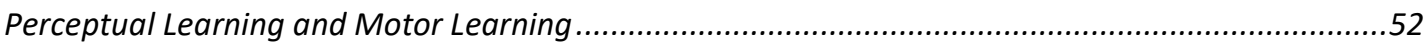

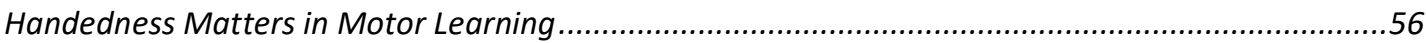

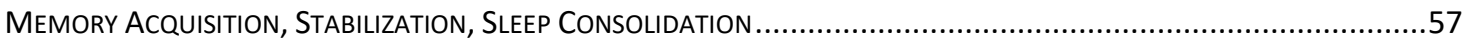

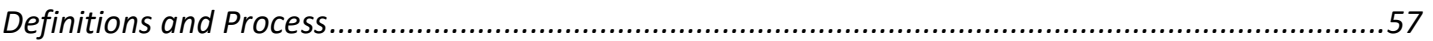

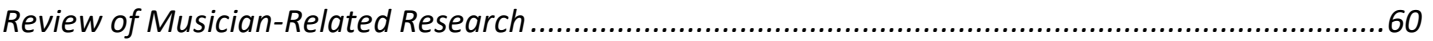

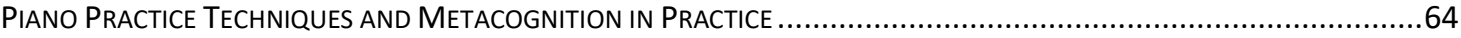

Practice Processes Observed in Highly Successful Pianists ....................................................64

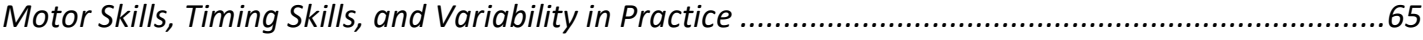

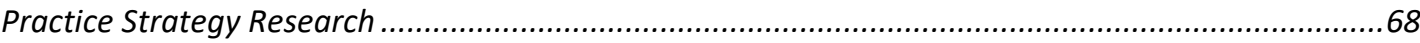

Organizational Goals, Practice Structure, and Metacognition ..................................................70

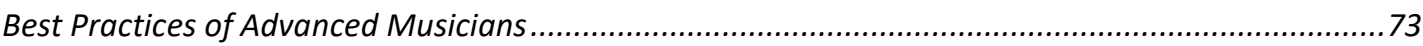

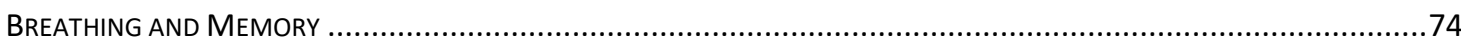

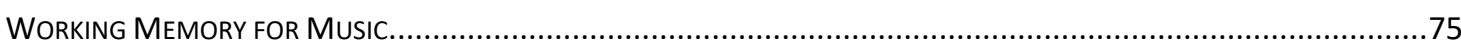

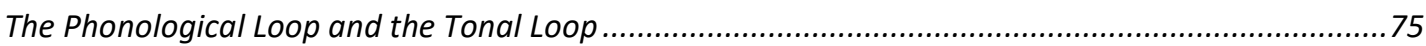

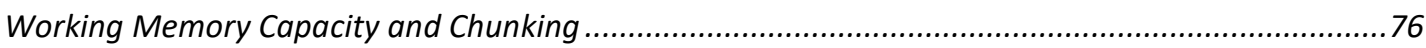

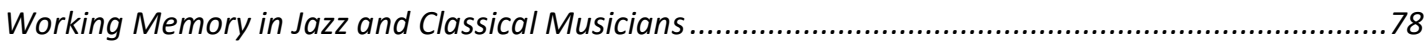

Different Attention Mechanisms Available to Working Memory ....................................................78

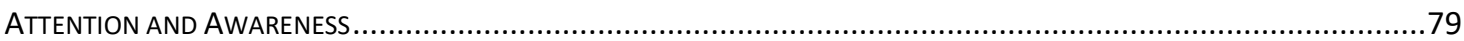

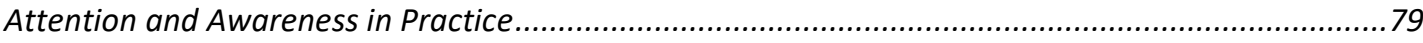

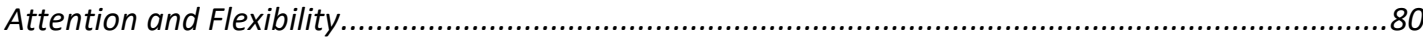

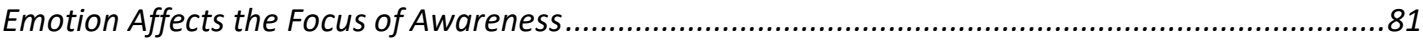

Selective Attention Works with One's Knowledge Base and with Working Memory .......................81

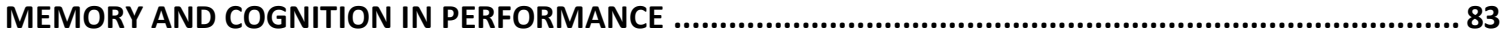

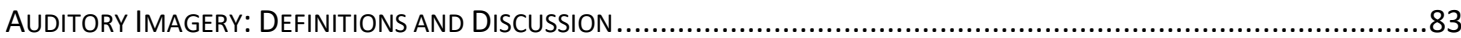

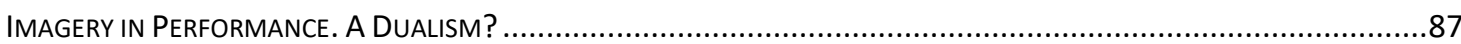

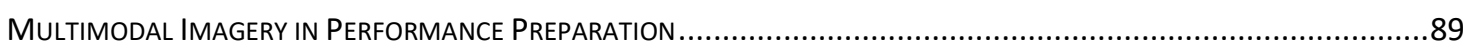

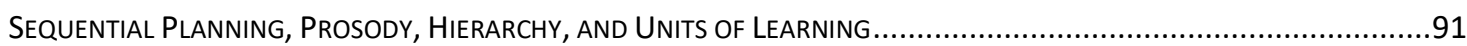

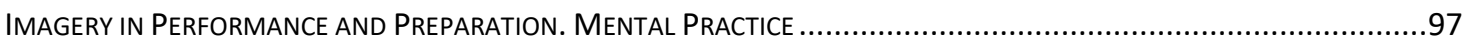

Working MEMory and Focus of AtTEnTION In Performance ......................................................100

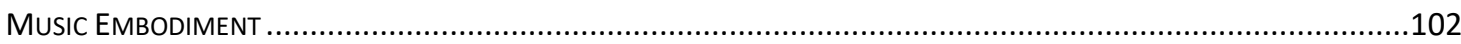

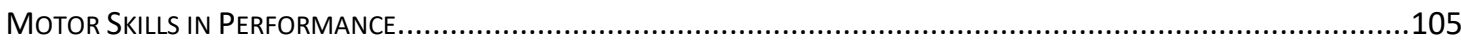




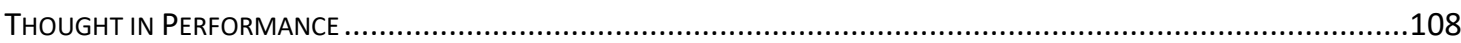

CONCLUSIONS AND THOUGHTS ON FUTURE RESEARCH .......................................................... 110

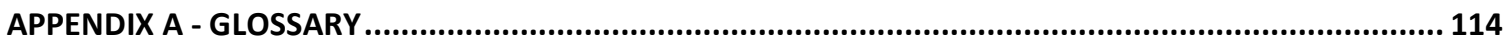

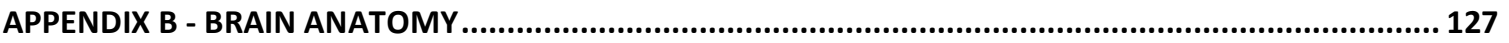

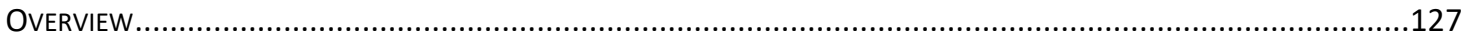

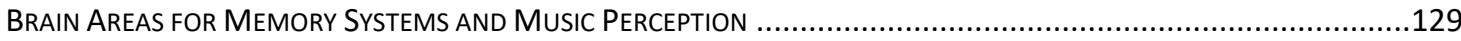

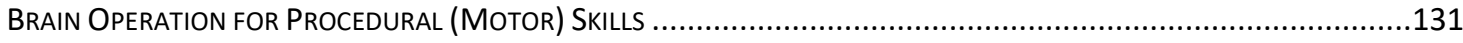

The DorSal ANd Ventral Streams In Auditory ProceSsing ...........................................................132

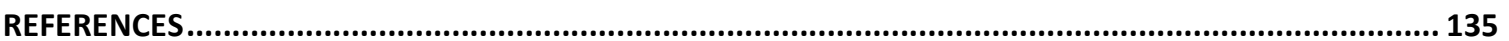




\section{Forward}

Roger Chaffin remarks that memorization [of piano music] has traditionally been looked upon as a method to be passed down from teacher to student, as an individualized process one must find for themselves, or as a process of sheer mystery (Chaffin et al., 2016). From the viewpoint of a pianist, nothing could seem to be more truthful. Yet, modern research has a lot to teach us about how people perceive, understand, and memorize music.

In her 1997 paper entitled, Music Performance, Caroline Palmer wrote that "distinctions between the psychological mechanisms proposed for music perception and performance are becoming blurred" (Palmer, 1997, p. 134). Bob Snyder concurs, adding in his book, Music and Memory, that "perception and memory are often impossible to separate; there may be little difference between memory processing and memory storage" (Snyder, 2000, p. 11). Indeed, the more information scientific study uncovers, the more music perception seems to be inextricably tied to the process of memorizing music, which in turn, underlies its performance.

This paper considers the perception, memorization, preparation, and performance of piano music as understood by current neuroscience research. 


\title{
Introduction. Memorization in History; Memorization Today
}

\author{
Music Memorization in Performance: A Brief History
}

In Jennifer Mishra's 2016 article, Playing from Memory, she explains that piano memorization became fashionable in performance during the middle of the $19^{\text {th }}$ century. While there is evidence that many great musicians like Mozart, Mendelssohn, and Paganini played from memory well before that time, memorization had neither been common nor encouraged before 1850. Instead, focus was placed on a performer's sight-reading and improvisational skills (Mishra, 2016, pp. 12-13).

Mishra recounts the episode in which Beethoven was not pleased when Czerny, at age 14, played one of his sonatas from memory for Prince Lichnowsky in 1805 (Mishra, 2016, p. 12). Primarily, Beethoven was concerned that the details of his music would be forgotten or, especially, misinterpreted. At the time, the dangers of forgetting music during performance were prevalent in the minds of performers (2016).

Performing from memory, as a movement, originated in the late 1830 s and 1840 s, with Clara Wieck's 1837 memorized performance of Beethoven Sonata op. 57 serving as an important impetus (Mishra, 2016, p. 12). In the 1830s, virtuosic, memorized performances by Wieck, Paganini, and Mendelssohn led the way. Liszt soon followed suit, performing mostly from memory by 1840 (Mishra, 2016, p. 13).

Mishra (2016) outlines two primary reasons for the rise of memorization at this time. First, music became more popular among the general population, who became interested in hearing and seeing virtuosity in performance (Mishra, 2016, p. 13). Amateur musicianship became common; it was stylish to have a piano and to be able to play it. Interest in thrilling, live performances grew, and more emphasis became directed toward individual performers like Liszt and Paganini (2016). While performers still improvised, they began to use memorization alongside virtuosity as a way to build their reputations (Mishra, 2016).

Second, the major works of important composers became formalized, standardized, and revered by students, amateurs, and professionals (Mishra, 2016, p. 13). At the time, Mendelssohn was instrumental in reviving music that had been considered to be out of vogue. Music of Bach, Handel, Mozart, and Beethoven became part of a respected canon of repertoire. Studying and hearing standard works by great composers became the norm. At this point, it became unacceptable to change or to improvise on the standard works. Memorization added a factor of virtuosity at a time when the 
performer's focus shifted toward being true to the exactness of a composer's work (Mishra, 2016, p. 13).

Interestingly, pedagogues of the era continued to discourage memorization until the turn of the $20^{\text {th }}$ century, in their pedagogical writings or directly in lessons (Mishra, 2016). Mishra shares that Chopin was angry when one of his students intended to play his Nocturne op. 9, no. 2 from memory and did not bring the score to the lesson. "I don't want any of this: are you reciting a lesson? I want to teach you precisely or not at all" (Jean-Jacques Eideldinge, 1987, as cited in Mishra, 2016, p. 13).

In the 1860 's and 1870 's, memorization was still thought of as a remarkable feat, but as it became more fashionable, performers feared that audiences would know if they made a mistake (Mishra, 2016). On this topic, Anton Rubenstein said, "I have been conscious of a growing weakness.... The public has always been accustomed to see me play without notes, for I have never used them; and I will not allow myself to rely upon my own resources to supply the place of some forgotten passage, because I know that there will always be many among my audiences who, being familiar with the piece I am performing, will readily detect any alteration. This sense of uncertainty has often inflicted upon me torture only to be compared with those of the Inquisition, while the public listening to me imagines that I am perfectly calm" (Rubenstein, 1902, as cited in Mishra, 2016, p. 14).

According to Mishra (2016), in the 1880s the practice of memorization in performance rose until everyone, including amateurs, was doing it. By the turn of the $20^{\text {th }}$ century memorization was commonplace. Focus then shifted from whether to memorize, to how to memorize. Both pedagogues and psychologists took up the cause (Mishra, 2016, p. 14).

The highly influential pianist and teacher, Theodor Leschetizky, may have been the first to develop a system for teaching memorization. Mishra (2016) explains that Leschetizky promoted a slow, concentrated mental memorization process that encouraged learning one measure or phrase at a time carefully before adding another. "Any one with the power of concentration can learn to play by heartno matter how intricate a composition may be" (Annette Hullah, 1906, as cited in Mishra, 2016, pp. 1415).

Mishra shares that an early and very comprehensive method for memorization was included in Frederick Shinn's 1898 book, Musical Memory and its Cultivation (Mishra, 2016, p. 15). The book contains information on four types of memory: musical (aural), visual, muscular, and intellectual memory. Philosophies of pedagogues and psychologists alike revolved around segmenting the music and memorizing in these four different ways. The form set by considering aural memory, visual memory, motor memory, and musical analysis has well withstood the test of time (2016). 
By 1900, the corresponding philosophy arose that music was not fully learned, and could not be fully interpreted, until a piece was memorized (Mishra, 2016, p. 15). The underlying idea that there is something of lesser quality to a performance that is not memorized remains to this day. Today, current thought has progressed such that memorization may be deemed natural if one has prepared properly to master a difficult piece.

\section{Memorization Today: The Significance of Music Cognition Research}

New research techniques have provided the unprecedented ability to learn about music comprehension and memorization by studying the brain while it is perceiving, learning, remembering, composing, and performing music. The study of music cognition has grown exponentially since the turn of the $21^{\text {st }}$ century, due to these new neuro-imaging techniques (Levitin, 2007, p. 11).

In a 2019 article, Hernandez-Ruiz surveys some of the new scientific methods, including structural magnetic resonance imaging (MRI), functional MRI (fMRI), magnetoencephalography (MEG), and transcranial magnetic stimulation (TMS) (Hernandez-Ruiz, 2019). Hernandez-Ruiz states that it is also common to use results from electroencephalogram (EEG) and other physical tests, like respiratory rates, to support research (Hernandez-Ruiz, 2019, p. 317). (See Appendix A.) While the empirical details of these tests are often excluded from the body of this paper, they form the basis for many of the conclusions described here.

Neuro-imaging techniques allow for observation of the entire brain in real-time situations, allowing for the examination of different portions of the brain, as well as various brain networks, while sensory-motor systems are at work in conjunction with cognition (Hernandez-Ruiz, 2019). Scientists look for electrical and chemical changes, along with their timings, such as ERP and MMN responses to explain brain function in various situations (Levitin, 2007). (See Appendix A.) These techniques have provided a new window into how humans learn, and how humans learn music. Specific studies in piano learning and performance have added to the body of knowledge.

The understanding of human learning techniques, human memory, and individual differences may provide the potential for optimized and specialized methods of learning music (Zatorre, 2013, p. 589). Because the complexity of piano performance requires many sensory systems working together with higher cognitive function, and the effects of the length of training on these systems can also be studied, the art of piano memorization, preparation, and performance is ripe for study (Herholz \& Zatorre, 2012, p. 486). A great deal of information is now available about music perception, learning, 
memorization, and performance, including knowledge about the acquisition and recall of the procedural motor skills required by pianists. The following is a compendium of current knowledge and research. 


\section{Human Memory Systems and Music: Concepts and Current Perspectives from Psychology and Neuroscience}

This section presents underlying background material for non-scientists to understand a platform from which neuro-music research has grown. It is intended to provide the reader with fundamental information and context from the fields of psychology and neuroscience for a better grasp of the meaning and importance of the research to be presented here. Further, some recent research included in this paper extends the knowledge presented in this section, or presents alternate, overlapping, or more specific theories of how memory systems might operate. In these cases, I refer the reader to later portions of this document.

\section{Theories about Human Memory}

\section{General Memory Theories: The Record-Keeping Theory and the Constructionist Theory}

In Foundations of Cognitive Psychology: Core Readings, compiled by Daniel Levitin (2011), R. Kim Guenther describes two classic theories in cognitive neuroscience that seek to explain the purpose and structure of human memory: the record-keeping theory and the constructionist theory. The goal of the record-keeping theory is that memory should preserve the past. In this paradigm, Guenther explains that memory is thought of as a storage area, much like a library, where memories are recorded and physically stored in a particular place to be revisited later. New memories fill new memory locations, increasing the number of books in the library (Guenther, in Levitin, 2011, p. 275). From this viewpoint, remembering is about searching through brain locations for a particular memory which can then be revisited in the mind. Difficulty in remembering is a result of interference during a search due to the large number of memories (2011).

\section{The Constructionist Theory.}

An alternative theory, as explained by Guenther, is the constructionist theory (Guenther, in Levitin, 2011, p. 275). The constructionist theory holds that memory's prime purpose is to anticipate the future. Instead of a record-by-record account of every experience, new experiences initiate changes in one's memory systems as one continually learns and adapts to one's environment. Guenther further explains that generalized information about the relationships between ideas, things, or events is kept, and is continually updated, but details are typically not retained. Instead, the brain remembers 
abstractions or generalizations about its environment, and one remembers those structural rules while letting go of the details. Therefore, remembering is based on reconstructing the past, based on our generalized knowledge and one's current experience, in concert with the contents and workings of our memory systems. Guenther supports the constructionist theory, suggesting that it is currently the most influential, and that it "more closely reflects what is known about the neurophysiology of learning and remembering" (Guenther, in Levitin, 2011, pp. 311-2).

One may intuitively see problems that each of these two models present. For example, in the constructionist theory, why do people have memories that include very detailed information, at least some of the time? In the record-keeping theory, why do people not run out of memory space?

\section{Multiple-trace memory models.}

Daniel Levitin, in his classic book, This Is Your Brain on Music (2007), discusses a set of hybrid theories that are now emerging, known as multiple-trace memory models. In these models, Levitin describes that every experience is encoded as a trace in memory, and with great accuracy. Both details and relationships are retained; however, information is recorded in groups of neurons in the brain, not in a particular place (2007). When these neurons become activated, one can replay a memory in one's mind (Levitin, 2007, p. 165). While multiple-trace memory theories currently cover a spectrum of detailed explanations, the central idea is summarized by Levitin in his chapter, "Memory for Musical Attributes" in Foundations of Cognitive Psychology: Core Readings: "Memory abstracts general rules from specific experiences, and it preserves to a great degree some of the details of those specific experiences" (Levitin 2011, p. 272).

Because multiple-trace memory theories are still emerging, the more favored constructionist viewpoint underlies the explanation of human memory systems in this paper.

\section{Human Memory Systems}

\section{Introduction to Human Memory.}

In his book, Music and Memory (2000), Bob Snyder explains that Long-Term Memory (LTM) can be thought of as a set of learned experiences that remain dormant and are generally not part of active awareness, or active consciousness at any given time. LTM does not act as a repository of information, like computer memory storage, that can be accessed at any time with its exact contents true and intact. Instead, Snyder explains that long-term memories are revisited and reconstructed in relation to a 
current experience or cue that has caused some of LTM contents to become activated (Snyder, 2000, p. $5)$.

\section{Encoding.}

Snyder defines encoding as the creation of long-term memories (Snyder, 2000, p. 72). Encoding happens in LTM when electrical and neurophysical changes take place in neurons (the nerve cells of the brain) that permanently strengthen their connections (Snyder, 2000, p. 4). In this way, encoding creates what we think of as a memory. A memory is sometimes referred to as a trace or an image (Snyder, p. 23; Roediger \& Guymn, in Bjork, 1996, p. 197). Snyder explains that the idea of encoding information also suggests that there is a method for translating it. According to Snyder, then, remembering a memory is more concerned with reconstructing information rather than simply reproducing it (Snyder, 2000, p. 72).

\section{What we already know.}

Snyder continues to explain that memories are based on, and largely constructed from, information that we already know. "Even quite novel events are usually experienced as deviations within some kind of framework of preexisting knowledge" (Snyder, 2000, p. 72). The parts of an experience that are most relevant to a person help to determine how much and what is remembered. Further, Snyder holds that everything one experiences and remembers is part of a context. Everyone has their own context memories, including both unique, individual knowledge, and shared cultural knowledge assimilated from one's environment. Snyder specifies that our memories for music are constructed from aspects of music we have already heard, and from other knowledge or experience about music that we already possess (Snyder, 2000, p. 72).

\section{Long-Term Memory - General information.}

Snyder (2000) suggests that the purpose of Long-Term Memory (LTM) is not about remembering the past; rather, it allows us to "adapt to a constantly changing environment" (Snyder, 2000, p. 71). Its workings are about adaptation, safety, and facilitating current and future awareness. Snyder explains that, continually, portions of LTM that are normally dormant become activated at a variety of levels when they are needed or cued. These activations work together with short-term memory (STM) to process our environment. LTM is dynamic. It can be, and regularly is, altered by its own processes (Snyder, 2000, pp. 71-2).

\section{Short-Term Memory - General information.}

Short-Term Memory (STM) involves information available in the short term, within an average time window of three to five seconds (Snyder, 2000, p. 9). According to Snyder, short-term memory helps us to process the present, but its contents cannot be remembered beyond the boundary of five 
seconds without rehearsal. For example, one may repeat an unknown phone number to remember it while walking toward a telephone; after its use, the number is quickly forgotten unless it continues to be rehearsed.

\section{STM Operations.}

Encoding, the making of permanent memories, does not happen within STM (Snyder, 2000, p. 47). Snyder shares an analogy that STM processing is like a pattern of electrical energy that reverberates in neural circuitry, but does not become permanent (Snyder, 2000, p. 47). Information persists for a short time, keeps its time order, is related to other information in STM, and then fades (Snyder, 2000, p. 48). Persistent information, for instance information that is meaningful to the individual or to the current experience, is continuously related to the newest inputs that enter STM. Events in STM can be immediately recalled as being connected. Importantly, events in STM also remain in a particular time order. Therefore, STM helps us to perceive present events as being continuous (Snyder, 2000, p. 48). The contents of STM are immediately available to our conscious awareness at any given time (Snyder, 2000, p. 48). (See the section on Working Memory for Music, p. 74 for more information.)

\section{Echoic Memory and Early Processing}

\section{Echoic Memory.}

According to Snyder (2000) echoic memory is part of our first level of immediate, auditory processing, often referred to as early processing. It occurs during the first, brief experience one has with music. Early processing creates a sensory representation of what is heard without any involvement of language areas of the brain. Instead, these memories consist of "imagelike abstractions" that cannot be explained in words (Snyder, 2000, p. 23). Echoic memory contains a large amount of auditory information for probably no longer than several seconds; it is heard like an echo (Snyder, 2000, p. 19). During this time, early processing determines what basic musical events have occurred. At this point, one perceives pitches, events that occur simultaneously, basic musical boundaries, intervals, dynamic changes, and timbre. Musical information recognized at this point is not yet processed and is still in continuous form (Snyder, 2000, p. 19).

\section{Early Processing. Event Fusion and Feature Extraction.}

Snyder describes the processes of event fusion and feature extraction as also occurring during early processing. During event fusion, events that happen closer in time than about 20 events/second fuse together to form pitches, even if more than one pitch occurred. In fact, chains of very similar acoustical events, not only pairs, will fuse to form a single sensation or pitch if the individual events are 
separated by less than $50 \mathrm{msec}$. (1/20 sec) (Snyder, 2000, p. 5). Changes in perceived frequency then form boundaries that tell the brain a different pitch event has been heard. According to Snyder, boundaries are concepts that the brain perceives to determine the beginning and ending of musical events or structures, and they are defined by various degrees of change that the brain perceives (Snyder, 2000, p. 32).

\section{Feature extraction.}

The auditory system contains specialized groups of neurons that detect musical features like pitch, continuous frequency changes (like slides, dynamics, overtone complexes), and changes in these phenomena (Snyder, 2000, p. 4; Levitin, 2007, p. 85). Snyder explains that feature extraction occurs when these so-designed groups of neurons recognize their intended features; they are detected, identified, and processed such that they are known to exist, and that they have beginning and end points. Both event fusion and feature extraction are brief, primary processes (Snyder, 2000).

\section{Short-Term Memory and Grouping}

\section{Short-Term Memory (STM).}

Snyder continues by explaining that information gathered from echoic memory and early processing becomes available for STM processing. STM works within its time limit of 3-5 seconds, while retaining the time order of the events it processes. At the STM stage, a more substantial grouping of musical information begins.

\section{Grouping and Perceptual binding.}

Grouping is a "natural tendency of the human nervous system" to build units of information that seem to be related (Snyder, 2000, p. 31). These units can be stored and remembered (Snyder, 2000, p. 32). In the process known as perceptual binding, similar events detected during event fusion and feature extraction become grouped together. Perceptual binding, also known as feature binding, is simply the STM process of grouping auditory information that is perceived from lower levels of early processing. STM can group melodic and rhythmic patterns, and functional units like sequences, up to the level of a phrase (Snyder, 2000, p. 37).

\section{Grouping factors.}

Grouping may have various bases. Grouping may happen based on proximity, such that events close together in time within STM will tend to be grouped together (Snyder, 2000, p. 39). Levitin (2007) also explains that the simultaneous timing of onsets is an important grouping factor. People can detect differences in timing of onsets as short as a few milliseconds (Levitin, 2007, p. 80). Even if timbres are 
different, sounds that happen exactly simultaneously will tend to be grouped as coming from a single sound source. Another grouping factor concerns spatial location. Because human audition can detect a sound's location, sounds that are perceived to be from one location are assumed to have the same source (Levitin, 2007, p. 80).

Levitin explains that, through adaptation, humans have evolved to comprehend that components of the harmonic series are likely to have come from the same source (Levitin, 2007, p. 79). Therefore, the harmonic series is a strong grouping factor, even allowing nearly all untrained musicians to be able to hear if music is coming from two different instruments (Levitin, 2007, p. 79).

\section{Similarity.}

Similarity is the concept that musical events that occur vertically together, like chords, and events that happen horizontally, near to each other in time, will be perceived as a group if their content sounds similar in some way. Levitin explains that timbre may be a similarity, in the sense that a group of violins may sound like one source to most people, because the individual violin sounds are so similar (Levitin, 2007, p. 77-78). Frequency is an important grouping factor, such that sounds with similar frequency ranges are assumed to have the same source (Levitin, 2007, p. 80; Snyder, 2000, pp. 40-42). In sum, features that are similar in some way, features that happen close together in time, or both, are perceived as having a single source, and are thereby grouped together. Related events are correlated and become units or auditory events (Snyder, 2000, p. 21).

\section{Continuity.}

When a series of continuous events changes in a particular direction in units of similar size, or if different directional changes use similar units, these events will tend to be grouped (Snyder, 2000, p. 43). Melodic groupings occur when pitches are similar in range, have patterns of rising or falling, or these observed patterns reverse. Rhythmic groupings occur when there are similarities in timing, accent pattern, or intensity (Snyder, 2000, p. 43). While a single grouping cannot exceed the time limit of STM, (Snyder, 2000, p. 36), the function of grouping is hierarchical, beginning in early processing and continuing through the highest levels of memory processing (Snyder, 2000, p. 33). Levitin also confirms that similarities in pitch or pitch change, or similarities in timing and accent patterns create good continuity. Good continuity is a factor for grouping (Levitin, 2007, p. 78).

\section{Grouping Boundaries and Example.}

Snyder explains that boundaries for STM groupings are defined by degrees of change one perceives in melodic motion or pattern, or rhythmic intensity or pattern. "A simplified example of perceptual binding might be combining the change that indicates the beginning of a note with its 
frequency and tone color ... to produce the impression of a coherent note starting at a particular time, with a particular frequency and tone color" (Snyder, 2000, p. 20). A melodic or rhythmic group boundary is usually determined by just one factor, like the duration of a pitch, but a phrase boundary usually requires more than one factor to be perceived. Combination factors that indicate the end of a phrase, at least in Western classical art music, might be a longer final note duration together with a slight pause after it (Snyder, 2000, p. 38).

\section{Primitive grouping. Bottom-Up and Top-Down Processes.}

Some grouping processes are innate and common to all human beings as a result of evolution. These are considered to be primitive grouping processes, also called bottom-up processes, because they act on stimuli entering our lowest levels of perception (Snyder, 2000, p. 32). We have no control over primitive grouping, wherein basic information like frequency and amplitude are processed during event fusion and feature extraction. Grouping factors at this level are almost all about sounds that come from a single source (Snyder, 2000, p. 34). Conversely, top-down processes are purposefully chosen by an individual by reason, or they occur due to learned behaviors. (See pages $22,43,47,81,86$ for more information on top-down processes.)

\section{Grouping is hierarchical.}

Snyder (2000) explains that more than one melody or rhythmic group can happen within STM's time limits, so several groupings can cohere to form a higher-level group, for example, an entire phrase. Because grouping is hierarchical, when several grouping factors happen at the same time, this often leads to a boundary in a higher-level grouping (Snyder, 2000, p. 43). In music, a phrase is believed to be the largest structure that can be understood within STM (Snyder, 2000, p. 36). Grouping processes in STM reduce the once-continuous information to a relatively small number of discrete sets or groupings, which significantly reduces the size of the retained information (Snyder, 2000, p. 4). The brain has begun to understand what it has heard.

\section{Short-Term Memory Capacity and Chunking}

\section{STM Capacity.}

The limit of the capacity of STM is crucial, particularly because of its short time window. Snyder (2000) reports that STM content limit has been, arguably, found to hold seven different elements, plus or minus two. To be clear, STM capacity is somewhere between five and nine elements, with an average of seven (Snyder, 2000, p. 50). An element in STM can be any basic element, but it may also be an item 
of larger size, containing information itself, without increasing STM load. For example, a pattern or grouping that fits in the time limits of STM can have embedded repetition, yet be just one item. An item that embeds other information in this way is called a chunk (Snyder, 2000, p. 54). (See pp. 76-79 for more information on Working Memory operations.)

\section{STM Chunks.}

A phone number is simple example of an STM chunk. Snyder (2000) explains that one does not have to remember ten individual digits if the number is already known; it is thought of as one element. A second example might be a group of nonsense letters, "cvfesilx," which would be seven elements, as opposed to "cabaret," which is just one word. "Cabaret" would be one chunk. Therefore, one STM element, alone, can be a grouping with five-to-nine elements inside it. So, chunking can reduce the number of elements in STM and save memory space (Snyder, 2000, p. 54). The final upper limit of STM capacity is probably about five groups of five events each, believed because five elements is considered to be the lower overall STM capacity (Snyder, 2000, p. 55).

\section{Activation of Long-Term Memory and Interchange with Short-Term Memory}

\section{Activation of LTM.}

Snyder (2000) explains that the brain correlates the sensory concepts it has currently created to long-term memories, which then become activated. Long-term memories that have some relationship to what is currently being processed are triggered and become part of current processing in STM. These related long-term-memories are called associations (Snyder, 2000, pp. 4-5). This process happens continually; there is constant interchange between STM and LTM. Snyder (2000) also clarifies that activated LTM can be in a spectrum of states that vary in their strength, including very vivid LTM, actively-in-use LTM, semi-activated LTM, or low-level activated LTM (Snyder, 2000, p. 5).

\section{Information in STM adds to or replaces information in LTM.}

Further, Snyder (2000) explains that long-term memories in any higher state of activation can persist as current STM. If not displaced by something new, they will remain with STM contents for 3-5 seconds, then decay if not rehearsed. The longer that STM contents are kept active by intentional rehearsal, the more likely they are to persist and become LTM. When we consciously try to memorize, information is repeatedly cycled through STM, increasing the chance of creating permanent changes in LTM (Snyder, 2000, p. 5). 


\section{STM information can add to, or modify LTM permanently.}

When STM information is well-rehearsed, new, particularly novel, striking, or meaningful in some way, it can be passed to LTM to become encoded (Snyder, 2000, p. 5). This is especially true if the information is meaningful to the individual, that is, important to either one's survival or to one's interests or goals (Smith \& Kosslyn, in Levitin, 2011, p. 191). Therefore, knowledge already in LTM influences memorability (Snyder, 2000, p. 53). Information from STM that is permanently encoded in LTM can add to LTM, or it can modify similar LTM memories that were already stored, thereby replacing them permanently (Snyder, 2000).

\section{Perception and Memory are difficult to separate.}

Snyder observes that, "perception and memory are often impossible to separate; there may be little difference between memory processing and memory storage" (Snyder, 2000, p. 11). Portions of LTM are activated or semi-activated relatively often, and its interchange with STM creates constant changes. "Our memories constitute who we are, and who we are is always changing" (Snyder, 2000, p. 71).

\section{Focus of Awareness and Working Memory}

\section{The FOA.}

The most highly activated part of STM is called the focus of awareness (FOA), or the focus of attention. Snyder (2000) explains that the FOA is equal to our conscious awareness at any given time. All of the contents of STM are immediately available to our FOA, but the FOA is the only part of STM that is completely conscious. While the FOA has an even smaller capacity than STM, holding only 3 items for immediate awareness (Snyder, 2000, p. 50), modern models are emerging that begin to explain how working memory may be optimized (e.g., Akiva-Kabiri, 2009; Ding et al., 2018). (See pp. 77-78.) Rehearsing material brings information back into the FOA, inside STM, for repeated processing, keeping it active (Snyder, 2000, p. 5).

\section{Working memory includes STM, LTM, and Context Memory.}

Snyder (2000) explains that working memory holds both immediate perceptions in STM that are related and time-related to each other, and also the activated long-term memories that they incurred, whether highly activated or lower activated. Highly activated long-term memories have immediate association with the current STM perceptions, so they intermingle in working memory processing. 
Lower-level activations of LTM are thought of as extended memory associations, not as vivid as the highly activated ones, but still somehow related. Once partially activated, they have a higher probability of becoming more fully activated; they are said to be primed (Snyder, 2000, p. 70). Primed LTM forms a context for our awareness. Context memory is comprised of partially activated, primed long-term memories that provide guidance to our conscious, more vivid memory processing, while remaining unconscious, themselves (2000).

In Foundations of Cognitive Psychology: Core Readings, (Levitin, 2011), Ashcraft \& Klein explain that the focus of awareness is the portion of working memory to which one chooses to give attentional effort at any given time (Ashcraft \& Klein, in Levitin, 2011, p. 321). Attention guides working memory operation. (See pp. 79-82.)

\section{Working Memory and Perception.}

Levitin (2007) shares the idea that without working memory (WM), one cannot process what has just happened in the immediate past in relation to what is current and what might be expected (Levitin, 2007, pp. 116). He clarifies that immediate information must be relatable both to the immediate past, for meaning, and also to the immediate future, to form expectations. Therefore, without WM there can be no perceptible music nor speech. "That we be able to hold in memory a knowledge of those notes that have just gone by, alongside a knowledge of all other musics we are familiar with that approximate the style of what we're listening to. . . the latter establishes a context for what we are hearing" (Narmour, 1992, as cited in Levitin, 2007, p. 116-7).

\section{STM and Scales.}

Interestingly, Snyder (2000) notes that, the average element size of STM is seven, the same as the number of pitches in our diatonic scale. World scales currently in use contain five to nine notes, often seven. From a cognitive psychology standpoint, it is believed that, "scales exist to bring [total pitch] resources within the capabilities of short-term memory" (Dowling, 1978, as cited in Snyder, 2000, p. 140). In her classic text, The Psychology of Music (1999), Diana Deutsch has stated that the purpose of scale size is so that repetition patterns can function like rehearsal and enhance memory (Deutsch, 1999, p. 398). 


\section{Chunking, Cuing, and Context}

\section{Chunking.}

Groupings, or chunks created in STM, seem to be a way for STM and LTM to work together (Snyder, 2000, p. 54). A chunk is the largest-level grouping that STM can process at once and is used as the basic unit of communication between activated LTM and STM (Snyder, 2000, pp. 54-56). Therefore, chunking may help memories to solidify in STM and move to LTM. Musical phrases and Alberti bass patterns are good examples of STM-sized chunks. Like lower-level groupings, a chunk can be part of a larger chunk, and so on, creating hierarchies that become encoded in LTM (Snyder, 2000, p. 36).

\section{Cuing.}

As memory systems go about their work, connections are created and facilitated between groups of simultaneously activated neurons (Snyder, 2000). A neural event that activates an associated long-term memory may also activate another long-term memory associated with it, and so on. This process is called cuing (Snyder, 2000, pp. 69-70).

\section{Cuing chunks.}

When chunks move to LTM, the last element in a chunk may act as a recall cue for the next chunk. We seem to remember long, time-ordered sequences of information by this process of associative cuing. When memorizing a lengthy piece of music, for instance, the entire piece is not stored; only chunks of information and pointers between the chunks are stored. It is thought that when a sequence (that is, a musical passage or piece) is learned more thoroughly, larger chunks form from the original ones, making the larger chunk cues higher in order and more important (Snyder, 2000, p. 55). Therefore, music encoding may be hierarchical, and related to the structure of the music. Also, this may be why one cannot simply start playing a piece in just any given place (Snyder, 2000, p. 55). The structure of a piece affects its learning and recall at the most basic levels.

\section{Closure of chunks.}

Chunks have clear beginning and end points, and many factors, mostly concerning musical syntax or energy, likely contribute to marking the closure of chunks (Snyder, 2000, p. 61-66). These include: notes in proximity, similarities, silence, intensity, decreased intensity such as falling pitch, increased dynamics, dissonance, change in tone color or speed, or the perception of motion vs. stability. When music exhibits no clear patterns of closure it is much more difficult to remember. Snyder says such music may "exist in the present only" (Snyder, 2000 p. 66). Aspects of closure can vary in degree and can relate to hierarchies of closure that relate chunks to one another (Snyder, 2000, pp. 61-66). 


\section{Chunking and LTM overlay.}

Snyder (2000) describes that while STM is operating, memories and experiences from LTM are triggered and enter STM. Chunks of experience are integrated together in the connection between STM and LTM. This is a process of one's current perception overlaid with information from activated LTM (Snyder, 2000, p. 48). While LTM associations work in hierarchy, they may also be cued flexibly and freely; otherwise, one could become stuck in hierarchical networks of thinking (Snyder, 2000, p. 48).

\section{Cuing and context.}

Cuing can rapidly move through the brain, activating many associations at once (Snyder, 2000, p. 70). Memories that are primed form the context of currently activated memory, so that all of our experiences may play a role in what we perceive, and in what our new long-term-memories might become (Snyder, 2000, p. 70). Because context memories are not in current consciousness, it continually becomes clearer that "a large part of our mental activity at any given time remains unconscious" (Snyder, 2000, p. 5).

\section{Context and music.}

If one hears a reasonably complex piece of music in a style one knows nothing about, it is not likely that one will remember very much (Snyder, 2000 p. 72), because one relies upon current knowledge to make sense of incoming information. Higher levels of musical understanding are constructed by the listener's own experience, together with the current musical content. "The brain constructs its own version of reality, based only in part on what is there, and in part on how it interprets the tones we hear as a function of the role they play in a learned musical system" (Levitin, 2007, p. 114).

\section{Long-Term Memory}

Long-Term Memory (LTM) is thought to work with larger concepts, like the perception of sections of a piece, movements of a piece, or entire pieces of music (Snyder, 2000, pp. 14-15). LTM is necessary to understand the form of music. LTM can help a listener to distinguish the character and order of sections, and help one to know a location among those sections where one might be while listening. LTM helps one to determine musical meaning and function (Snyder, 2000, p. 14).

According to Snyder (2000), because STM operates on such a short time scale, any music longer than a phrase, or requiring more than 5 seconds in time, probably has to be reconstructed from LTM. While one may still have a sense of phrases heard in the recent past, as time progresses, these memories fade as they leave STM. So, when listening to a whole piece of music, one understands its 
parts and their relationships because they return to awareness from LTM, either during its interchange with STM or by conscious recollection (Snyder, 2000, p. 15).

\section{Feedback loop.}

Snyder (2000) suggests that, because LTM associations with new events are based on what one already knows, LTM may act as a filter of information, allowing only a small part of its activated associations to enter the FOA at any one time (Snyder, 2000, p. 11). In turn, conscious awareness also regulates what is persistent, i.e., what becomes important enough to be sent from STM to LTM. Because these controls work in both directions, they act like a feedback loop (Snyder, 2000, p. 11). One's experiences, values, memories, and goals in LTM determine the fraction of information that one is consciously aware of. This, in turn, determines what becomes stored in LTM. "What we already know literally determines what we see and hear, which means that we see and hear what we look for more than what we look $a t^{\prime \prime}$ (Snyder, 2000, p. 11). Snyder clarifies that this is similar to saying that consciousness is primarily "goal-driven" (Snyder, 2000, p. 17).

\section{Kinds of cuing.}

Cuing occurs when long-term-memories connected to our current perception are automatically activated, creating our basic awareness. Snyder discusses various types of cuing. "Reminding" happens automatically when we see or hear something familiar (Snyder, 2000, p. 70). "Recollection," or recall memory, happens when one intentionally tries to cue a memory. It is not automatic; it happens by choice (Snyder, 2000, p. 70). Finally, "recognition" occurs when an environmental event acts as its own cue (Snyder, 2000, p. 70). If an activated LTM is very close to, or perfectly matches a current experience, we "re-cognize" it (Snyder, 2000, p. 23). Both reminding and recognition are spontaneous, and they happen constantly.

\section{Recognition and habituation.}

A recognized LTM moves to a state in the background of perception, instead of into the FOA, because there is no need to process it again. This process of habituation is a part of human adaptation: the mind does not pay attention to things it already knows (Snyder, 2000, p. 23-24). The recognized, activated LTM is no longer at a conscious awareness level. Our attention generally leans away from things that are familiar and toward things that are novel, or that seem unstable or unpredictable (Snyder, 2000, p. 24).

\section{Keeping correct cues to a memory.}

LTM cuing can be interrupted by interference, or irrelevant cues that are similar to the correct cue (Snyder, 2000, p. 70). Because information in LTM must be cued and reconstructed, it may not 
always be easily accessible. Forgetting typically occurs when the associated cue is lost, rather than the memory itself being lost (Snyder, 2000, p. 70-71). Many researchers currently believe that, over our lifespans, we do not necessarily keep every single memory in LTM forever. As the brain is very efficient, information that is not frequently used may not be maintained as well as other memories. Therefore, memories can fade over time, and their neural networks may be recruited for information the brain deems to be more valuable.

\section{Categories}

In Foundations of Cognitive Science: Core Readings (Levitin, 2011), Rosch explains that categories are the highest level of hierarchy in human understanding. Categorization helps the brain to organize large volumes of information without becoming overloaded with unnecessary details (Rosch, in Levitin, 2011). Categorization is an important part of human adaptation and seeks to find the world as organized, not arbitrary or unpredictable (Rosch, in Levitin, 2011, p. 156). This prevents one from living in such a way where everything one encounters would be a first experience with it, which would be continually bewildering (Rosch, in Levitin, 2011, p. 156).

\section{Category examples and loss of detail.}

The concepts of what a tree is, what a bird is, or what a lamp is, are good examples of categories (Snyder, 2000, p. 84). We store information that defines a category and the similarities between examples that create a framework for its identification (Snyder, 2000). We also store primary examples of a category. Snyder details that, for instance, the fact that a robin is a type of bird, and that a robin is likely to be a quintessential example, makes a robin a prototype of the bird category (Snyder, 2000, p. 84). The brain remembers prototypes, examples, and rules about a category, but typically not the details of each time it has been experienced (Snyder, 2000). Smith \& Kosslyn also describe the workings of categorical knowledge at length in Levitin's Foundations of Cognitive Psychology, Core Readings (2011). They suggest that once one encounters a thing that belongs in a category, further knowledge about that category becomes available to them, depending on the situation at hand (Smith \& Kosslyn, in Levitin, 2011, p. 180).

\section{Multimodal categorization.}

In Foundations of Cognitive Psychology: Core Readings (Levitin, 2011), Smith \& Kosslyn discuss that categorization of information happens across six sensory modalities: vision, audition, action, touch, taste, and smell. Psychologists refer to this kind of process as being multimodal. This is how people 
under normal conditions (for instance, people without synesthesia) may sense colors, textures, and spatial actions like rising and falling when listening to music (Smith \& Kosslyn, in Levitin, 2011, p. 200).

\section{Categorization in all processing levels.}

Levitin (2007) reminds us that categorization breaks our experience into discrete parts for processing and remembering, from early processing, to chunking, to higher categorization. Even knowing that basic musical events happen in relation to background noise is a kind of categorization (Levitin, 2007, p. 101). Our ability to hear many different sounds at once via grouping is amazing. "No one has yet designed a computer that can perform this [the] task of sound source separation" (Levitin, 2007, p. 101).

\section{Music category examples.}

In music, people develop categories for musical intervals, and for the roles of pitches in a scale. In 1990, Krumhansl et al. showed that people understood principles of hierarchy of the tones in a scale, and the intervals they form, simply from the passive exposure to music in one's culture. Trained musicians can identify intervals they hear, even if they are not mathematically exact, because they are learned conceptual categories. The minor changes in pitch that are not interruptive to perception are considered to be nuance (Snyder, 2000, p. 86).

Rosch is credited for showing that rules about a category are not absolute; instead, there is a spectrum along which things may belong (Levitin, 2007, p. 143). According to Rosch, something can be "more or less" a category member, rather than having absolute membership or not. For example, one might say, "a penguin is technically a bird, but it can't fly like most birds do" (Levitin, 2007, p. 143). The brain manages conflicting information by selecting the most likely choice according to what the environment is presenting.

\section{Types of Long-Term Memory}

Long-Term Memory (LTM) is typically divided into two types: implicit memory and explicit memory (Snyder, 2000). These two types engage different parts of the brain.

\section{Implicit memory.}

The first, implicit memory, is not available to consciousness, and one is not aware when it is at work. It is often impossible to describe in words (Snyder, 2000, p. 72-73). Implicit memories are likely the only kind that humans can have before acquiring language. Currently, it is thought that there are many kinds of implicit memory. For example, priming is thought to be a type of implicit memory because 
priming is the ability of previous experience to affect recall without any conscious awareness that it is happening (Snyder, 2000, p. 73). Assimilating music into memory requires the context provided by implicit memory; therefore, implicit memory is necessary for understanding music.

\section{Procedural memory.}

One of the types of implicit memory, procedural memory, is roughly the same as a skill, or how to physically do something, like riding a bicycle or playing the piano. Procedural memory is formed through laborious, repetitive practice (Snyder, 2000, pp. 73-76). It is not very flexible. It can be refined only slowly and is difficult to change radically. However, it is quickly and automatically recalled without conscious effort. In fact, conscious effort often impedes the use of implicit memory. Procedural memory is usually expressed by performing the skill, rather than by recollection or verbal description (Snyder, 2000, p. 73).

\section{Explicit memory.}

Explicit memory is used when we consciously memorize or recall information. It is memory of events or concepts that can be described in words (Snyder, 2000, p. 74). Explicit memory includes music or lists of things we memorize. Also, it is how we know that an event took place, or that something is factual. Explicit memory may be established quickly, but it also requires conscious effort to use it and recall it (Snyder, 2000).

Explicit memory is believed to have two kinds, episodic memory and semantic (declarative) memory. Episodic memory is the encoding of specific experiences or events, that is, autobiographical memory that always involves the presence of self (Snyder, 2000, p. 75). Episodic memory is created very quickly and is recalled in a time order (2000).

Episodic memories are highly susceptible to distortion, especially with repeated recollection, because the memories are changed by the very process of recollection (Snyder, 2000, p. 75). Recalling a memory about self is an episode, itself; then this "copy" of the memory replaces the original one. This process has a bearing on how we represent things to ourselves and it interacts with our values and what is meaningful to us (Snyder, 2000, p. 75). (See p. 22 for information on semantic memory.)

\section{Schemas and loss of details.}

Eventually we lose the details of episodic memories, but an abstract template or schema is derived from commonalities in all the related experiences (Snyder, 2000). A schema is a kind of categorization for types of situations, or for processes or procedures. Schemas function as sets of ideas about how things usually are (Snyder, 2000, p. 95; Smith \& Kosslyn, in Levitin, 2011, p. 210). General examples include: what it means to go to a restaurant, to travel by airplane, or to make a telephone call. 
Schemas, like categories, are top-down groupings on a large time scale that are learned (Snyder, 2000). They are based on an individual person's previous experience and culture (Snyder, 2000).

\section{Musical schemas.}

"Schemas provide frameworks within which to evaluate novelty and thereby guide attention" (Bregman, 1990, as cited in Snyder, 2000, p. 96). People have schemas that abstract the understanding of musical patterns and musical styles. "Schemas in the form of musical patterns and styles are largely responsible for our feelings of expectation while listening to a piece of music" (Snyder, 2000, p. 96). Melodic schemas help us to perceive melody. Motor schemas are critical for performing musicians (2000). (See pp. 49-56 for a full explanation of generalized motor programs and motor schemas.)

\section{Schema examples in musical form and style.}

Snyder suggests, as an example, that the theme-and-variation form is based on using schemas. Primary material is first presented, like a template, then varied such that the relationship between the variation and the theme can usually be heard (Snyder, 2000, p. 101). Jazz improvisation that is based on harmonic structure is a good second example, because the improvisation requires constant comparison with the basic schema structure (Snyder, 2000, p. 101).

\section{Semantic memory.}

Semantic memory, the second kind of explicit memory, is the conscious memory of facts or concepts. It is involved in recognition, and is often thought of as a set of learned information. Semantic memory is used to consciously memorize music (Snyder, 2000, pp. 77-78). Since we generally process information through semantic memory categories, the category information is retained, and details are not. In Levitin's Foundations of Cognitive Psychology: Core Readings, Ashcraft \& Klein describe how concepts that are regularly used in semantic memory can be learned so well that they become automatic (Ashcraft \& Klein, in Levitin 2011, pp. 348-349). Therefore, for the detailed memorization of music, practice and repetition are necessary to create automaticity, along with other preparation methods to be described here. (See pp. 24-25.)

\section{Nuance.}

Snyder (2000) explains that one's musical memories can also be categorized. Categories range from the sounds of a specific instrument, to hierarchies for intervals and tonal structure. Musical variation that happens inside the boundaries of a musical category is called nuance. We notice nuance's variations, but nuance does not affect the basic category that we perceive (Snyder, 2000, pp. 87-88). Nuance exists in echoic memory, but is lost in upper-level processing, with the result that we experience it, but do not remember it well. Nuance includes many phenomena: subtle bending of pitch, vibrato, 
intonation variations, small rhythmic inequalities, rubato, colorations, and individual timing and expressiveness in interpretation (2000).

\section{Nuance, recordings, and notation.}

Our ability to perceive nuance explains why detailed events in musical recordings, for instance, still seem interesting and important after numerous hearings, and why we enjoy hearing many different performances of the same music (Snyder, 2000, p. 88). Notation captures only the aspects of music that remain the same among different performances, but it cannot capture nuance. "If nuance could be captured in notation, it would take almost an infinite number of symbols to do it" (Snyder, 2000, p. 88).

\section{Auditory Scene Analysis and Streaming}

\section{Auditory scene analysis.}

Auditory scene analysis is the study of how we take multiple auditory inputs and determine what we are hearing. It is an amazing feat of human audition and perception that one can make sense of a complex set of auditory surroundings. According to Bregman, "the job of perception, then, is to take the sensory input and to derive a useful representation of reality from it" (Bregman, in Levitin, 2011, p. 123). The way one's nervous system groups sensory inputs determines the patterns one perceives (Bregman, in Levitin, 2011, p. 124). Auditory scene analysis is mostly about determining which sounds came from the same source (Snyder, 2000, p. 34). In other words, it is primarily about streaming.

\section{Streaming.}

Snyder explains that a stream is an acoustical image constructed by the brain of a coherent sound source (Snyder, 2000, p. 144). The sounds might be simultaneous or sequential, but they come from a single source. Streaming is an example of the categorization of sound sources (Snyder, 2000, p. 144). Grouping factors for streaming are the same as those described earlier for STM chunking. (Snyder, 2000, pp. 143-145). (See pp. 11-12.)

\section{Multiple streams.}

If pitches are far enough apart in frequency or time, the brain assumes they are from different sources, and separate vertical groupings are made. Streaming, then, allows one to hear different sounds at the same time and to identify them as coming from different sources (Snyder, 2000, p. 144). One can perceive the presence of up to about four multiple streams, although one can only attend to one at a time (Snyder, 2000, p. 144). 


\section{Habituation and streams.}

Streams that contain different sorts of repetitions can be heard as foreground, middle-ground, or background. One can be heard as being "in front of" another, such as a melody that is heard "over" an Alberti bass (Snyder, 2000, p. 145). This is also related to habituation, because we pay less attention to things that are familiar. Therefore, our attention is guided toward some streams more than others.

\section{Attention and Expertise}

\section{Attention.}

In Levitin's Foundations of Cognitive Psychology: Core Readings, Ashcraft \& Klein discuss attention as being the mental process of intentionally focusing on an activity, such as memorizing music (Ashcraft \& Klein, in Levitin, 2011, p. 321). Attention is "the limited mental energy. . . that powers the mental system, ... the stuff that gets focused when we pay attention" (Ashcraft \& Klein, in Levitin, 2011, p. 322). In other words, paying attention means deliberately choosing to use one's concentration for a particular activity.

\section{Attentional Limits.}

Ashcraft \& Klein continue explaining that the amount of attention that can be given to any one task has limits, as does the quantity of things that can be given our attention at the same time (Ashcraft \& Klein, in Levitin, 2011, p. 322). There is a physical limit to the capacity of the FOA; however, some automatic tasks can be performed with little or no attention (Ashcraft \& Klein, in Levitin, 2011, p. 346). Significant amounts of practice or knowledge make some tasks easier and less demanding on our attentional resources, also known as cognitive load.

\section{Attentional resources.}

Attentional resource theories vary. However, it is thought to be most likely that one can only attend to one concept or detailed activity at a time (Ashcraft \& Klein, in Levitin, 2011, p. 345). If we are doing or considering more than one thing at a time, we are actually alternating between the two, unless one of the processes is automatic. Automatic processes can happen without intention and use little or no conscious resources (Ashcraft \& Klein, in Levitin, 2011, pp. 345-346).

\section{Repetition, automaticity, memory as a requirement.}

Ashcraft \& Klein hold that automaticity has been shown to be attained by sheer repetition of practice, while paying attention to the stimuli involved and to the actual task. More than 40 years ago, Shiffrin \& Schneider (1977) concluded scientifically that old-fashioned, repetitive practice has the largest 
effect on automaticity (Shiffrin \& Schneider, 1977, as cited in Levitin, 2011, p. 348). Interestingly, after a process has become automatic, giving more explicit attention to the process can lead to poorer task performance (Ashcraft \& Klein, in Levitin, 2011, p. 352). Another downside to automaticity is that it can be difficult to change once it is achieved, requiring lengthy, concerted effort (Levitin, 2011, p. 351). Automaticity benefits greatly from overlearning, that is, practicing beyond mastery of the material, thereby increasing the number of correct repetitions (Levitin, 2011, p. 349).

\section{Expertise.}

Research also shows that facility in an area depends on prior experience in that domain. Levitin (2007) explains that the "more experiences we have with something, the stronger memory and learning traces for that experience become" (Levitin, 2007, p. 196). Memory strength is related to how many times a stimulus has been experienced. Therefore, expertise in the area of piano music is the most important factor one can have for memorizing the details of piano music. Further, memory strength is also correlated with how much we care about something (Levitin, 2007, p. 197). This is due to emotion aiding in neurochemical processes that tag information for further processing and memory consolidation (Levitin, 2007, p. 197). (See pp. 57-63 for information about memory consolidation.) Expertise, then, depends on experience, meaningfulness, and correct repetitions. Further, Levitin (2007) summarizes that "musical expertise is not one thing, but involves many components, and not all musical experts will be endowed with these different components equally. The process of becoming a musical expert ... requires many of the same personality traits as becoming an expert in other domains, especially diligence, patience, motivation, and plain old-fashioned stick-to-it-iveness" (Levitin, 2007, p. 220).

\section{Review and Summary}

In summary, human memory systems operate at various levels to process the auditory scene and analyze it. Low-level processing recognizes the fundamental aspects of what we hear, after which grouping processes begin. Short-term memory comprehends patterns and phrases; it also interacts with LTM associations to process what is heard. Broader LTM associations provide context for what is heard, which guides our understanding, while the most important or novel LTM associations are processed with STM to create new or updated permanent memories. Long-term memories are encoded as true memory traces. The differences between perception and memory are now considered to be blurred; therefore, the process of memorizing piano music is heavily dependent upon how we perceive the music. 
Higher-level comprehension requires processing in LTM; therefore, the concept of structure in music is inextricably tied to its memorization. Categorization, schemas, and all other forms of grouping allow the brain to process larger amounts of information without becoming overloaded. Schemas for music styles and for motor skills are crucial for pianists. Cuing allows pianists to remember lengthy sequential information by associative chunks. Automaticity in piano performance is nearly essential; toward that goal, extended over-practicing is highly beneficial. Ultimately, expertise in piano music is the most important factor in being able to memorize and perform piano music accurately. 


\section{Memorization of Piano Music}

\section{Conceptual Learning and Remembering}

\section{Understanding Structure. Score Analysis, Silent Learning, and Memorization}

Musicians and pianists with traditional training are accustomed to using scores, not only for physical practice, but also for theoretical analysis and study away from an instrument. These methods are very often used together to internalize music, to understand a composer's intentions, and to memorize music thoroughly. The research study described in this section sought to understand the individual roles of theoretical structural analysis and score studying in silence, as regards memorization.

In 2015, 89 musicians, including pianists, described the role of score analysis in their work during a study by Fine et al. (2015). Responses fell into three broad categories. The first had to do with learning the structure and aesthetic features of a piece, including its form, tonality, melody, and harmony. This included the desire to understand meter and rhythm, and where applicable, how the piano part fit into a larger work. When mentioned, aesthetics included the desire to understand dynamics, tempo, style, phrasing, and the context of the composition (Fine et al., 2015).

The second category of responses was related to defining a piece by breaking it into portions and studying its detailed construction (Fine et al., 2015). Participant responses included the desire to inspect a score visually prior to a first playing, in order to survey it for difficulties and to explore its texture. Some participants mentioned theoretical aspects of analysis, but only one mentioned audiation, hearing the piece inwardly while studying the score (Fine et al., 2015). (See pp. 33-35 for more information about audiation.)

The final category encompassed two main goals of score analysis: the realization and the interpretation of the music, including the location of technical difficulties and details that aid in interpretive choices (Fine et al., 2015). Score study is an important method for understanding the construction and musical goals of a piece. Quoting Hill from his research in 2002, score study is utilized so that performers may "liberate [their] musicality, to make sure that musical goals - not technical constraints-come first" (Hill, 2002, as cited in Fine et al., 2015, p. 76). (More results from this study regarding performance are discussed on p. 97.)

Does the style of music to be learned have any effect on score studying? In 2018, Loimusalo \& Huovinen researched silent memorization in pianists, comparing the memorization of tonal and nontonal music when pianists only studied a score. Their goal was to learn about strategies pianists use to 
memorize, and about the effects of musical expertise on silent memorization. To assess performance, they used a recall rate to determine accuracy of memorization in each hand, and they assigned a rating for the overall impression of fluency and style (2018).

Participants reported that memorization of tonal music was easier. The pianists used aural imagery, imagining the music in their minds, to aid with memorization. Especially, they imagined singing or hearing the right-hand melody in their minds, making it easier to remember. Some participants listened to the left-hand accompaniment, separately, in their mind, while others reported that they listened to the melody, but that they analyzed the accompaniment (Loimusalo \& Huovinen, 2018).

With atonal music, even advanced aural skills like absolute pitch did not prove helpful to participants (Loimusalo \& Huovinen, 2018). Participants all reported the ability to hear the melody well, but the structure was so unusual that they could not remember it (Loimusalo \& Huovinen, 2018). They tended to focus their aural imagery on rhythm instead of pitch. One participant admitted that she could only deliver the feeling of the piece, perhaps with slurring or articulation, because playing the correct notes was impossible. If the pianists tried to group notes by interval structure or possible chord names, the music itself did not support their strategy. Instead, participants commonly used sequential lists of letter names, or created gimmicks to help their minds remember anything that they possibly could (2018).

Overall, in tonal music, pianists imagined the melody and often analyzed the accompaniment, but typical strategies broke down for atonal music, so participants' attention shifted toward rhythm (Loimusalo \& Huovinen, 2018). On the whole, better score tallies for overall impression were correlated to musicians with better aural skills (2018).

On average, the participants reported using about four different memory strategies at a time, demonstrating that they chose to encode their memories into multimodal systems from the first, sightonly, silent rehearsal (Loimusalo \& Huovinen, 2018).

In summary, this research found that "analysis and imaginary practice were used flexibly side by side, and not as two distinct activities. Skills in pitch imagery and habitual practice in conceptual processing may guide musicians in their situationally chosen strategies. Such results also draw attention to the importance of musical experience and the effect of musical structure on memorizing" (Loimusalo \& Huovinen, 2018, p. 236). 


\section{Hear First, See First, or Play First? The Role of Modality in the Original Learning Process}

Musicians commonly ask whether it is advantageous to listen to a new piece first, or to physically learn it first. Research on the modality of learning new music is quite interesting. When music is learned by playing first, the brain's recognition ability and motor planning areas are positively affected. The full understanding of a brand-new piece, however, may rely on hearing the piece in full prior to practice. A first hearing has also been shown to increase practice gains and to improve the benefits of sleep consolidation. (See pp. 57-63 for more information about sleep consolidation.)

Mathias et al. (2016) researched the ability of pianists to recognize melodies that they learned either by hearing them or by playing them. After learning melodies via each mode, the melodies were played back to the pianists with occasional alterations such as incorrect, out-of-key pitches. The pianists were asked to identify whether the melodies matched the original melodies or not (Mathias et al., 2016, p. 1111).

By studying effects in the brain during the experience of altered pitches, this research found increased activity in cortical motor planning areas if the melody had been learned by playing it (Mathias et al., 2016). Further, these results correlated with pianists' detection accuracy in the retest. Performing melodies altered the brain's sensory and tonal recognition cues when hearing the music again, suggesting that "learned auditory-motor associations influence responses to out-of-key pitches" (Mathias et al., 2016, p. 1111).

Overall, the research found that "memory recognition for sound sequences may rely not only on sensory and cognitive auditory information, but also on the sensorimotor modality through which the sequence was encoded" (Mathias et al., 2016, p. 1123). This study suggests that the original modality of learning may be significant in how music is remembered in the long term (Mathias et al., 2016, p. 1123).

Other research suggests different ways of approaching the issue. Research performed by Dowling \& Tillmann (2014) suggests that, when beginning to practice a musical work of which one has little or no prior auditory knowledge, it is critical to hear the entire work of music prior to practicing (Dowling \& Tillmann, 2014). This study “investigated a memory improvement effect that has been observed for musical material occurring over delays filled with interfering material, namely, the continuation of the piece" (Dowling \& Tillmann, 2014, p. 28). In other words, they studied how "feature binding," the grouping of similar events detected during event fusion and feature extraction, interacts with the continued playing of a piece of music. By so doing, this study researched the amount of time 
required for feature binding, especially of the melodic contour, to make clear decisions about interpretation of pitch, timbre, loudness, and timing to be sent to the next processing level (2014). (See p. 10 for more about feature binding.)

When short intermittent delays occurred while listening to new music, listeners were confused about what they had heard, because feature binding had not yet related the melodic content to its tonal structure (Dowling \& Tillman, 2014). This research found that feature binding in complex music takes longer than feature binding for simpler musical events, requiring some number of full seconds (2014). More importantly, the simultaneous perception of the music while it continues to play was shown to be "essential to the continuation of the encoding of the earlier material and the binding of individual stimulus features into coherent memory representations" (Dowling \& Tillmann, 2014, p. 31). These results suggest that hearing a new work in its entirety is crucial to allowing its aural memory to be properly encoded (Dowling \& Tillmann, 2014).

In 2014, Cash et al. tested whether hearing a model performance of a piece prior to practice had any effect on overnight sleep consolidation. This 2014 study was one of the first to test immediate effects of hearing a model on the process of early acquisition of music (Cash et al., 2014, p. 90).

Experienced musicians were tested with the only criteria being the number of correct key presses. All participants made noticeable improvements after overnight sleep, but those who listened to the model made significantly larger gains than those who did not. Also, those who heard the model performance first had noticeably better performances in both the training session and the morning retest (Cash et al., 2014, p. 89).

The reasons for this outcome could not be exactly concluded from this study, but the results suggest that there are short-term advantages to hearing a model prior to practice (Cash et al., 2014). It was noted that experienced players tend to prioritize accuracy over speed in practice, which may inhibit or restrict early practice if a demonstrative model was not heard first (Cash et al., 2014, pp. 94-95). Further, it was posited that listening to a model performance may have "created an auditory template that primed the motor system prior to the start of active practice" (Cash et al., 2014, p. 95).

Overall, "the data demonstrate that hearing a model performance that is performed at a speed beyond what may be attained in a single practice session led experienced musicians to accomplish more during active practice and to benefit more from overnight memory consolidation than did musicians who did not hear a model" (Cash et al., 2014, p. 96). Much more research on the effect of hearing model performances on the performance of novices vs. skilled learners, as well as in early acquisition vs. longterm performance, is warranted. 
Individual people may favor visual or aural learning by choice. Therefore, the mode of original learning and the role of individual learning preferences in remembering unfamiliar melodies was studied by Korenman et al. (2007). The first of two tests studied learning efficiency for meaningful and lessmeaningful melodies. The second test studied memory for the same two kinds of melodies (2007).

Korenman et al. (2007) considered melodies to be meaningful if they followed conventional rules and fulfilled expectations; less-meaningful melodies did not fulfill these criteria. The lessmeaningful melodies were created from four original melodies by trading measures from one melody with another. For example, measure two from melody one became measure three of melody two, and so on. The less-meaningful melodies were finalized as two-measure versions of the mixed-up musical material. Meaningful melodies kept the original melodic material intact (Korenman et al., 2007).

Meaningfulness is an interesting variable in learning and memory, because it is already known that meaningful information is more easily learned and remembered (Snyder, 2000, p. 53). Korenman et al. (2007) pursued whether meaningfulness interacts with the original presentation mode of music. They shared that in prior research, "several studies have found that, in general, auditory presentation leads to better learning and memory than does visual presentation; however, they found that, although initial learning benefits more from auditory presentation, longer-term memory benefits more from visual presentation" (Korenman et al., 2007, p. 917).

Participants were allowed to choose a preference for learning style, based on scientific studies validating that people can be classified as visual or auditory learners (Korenman et al., 2007). Half the participants were visual learners, and half auditory learners; these categories were each comprised of half musicians and half non-musicians.

Results demonstrated that, both in testing for effects on learning and in testing for effects on memory, the original mode of engagement had no effect at all (Korenman et al., 2007). It was shown that the auditory learners were, indeed, better at hearing first, and the visual learners were better at seeing first. Further, musicians remembered more of the meaningful melodies than non-musicians did, and learned them faster, but meaningfulness did not help performance of non-musicians in the study (Korenman et al., 2007).

This test is fascinating, especially in light of more current knowledge that hearing an auditory model prior to practice has demonstrated a positive effect on learning. At minimum, there was clearly an effect demonstrated from experience, in that musicians were better able to memorize meaningful music (Korenman et al., 2007). However, it should be noted that this test did not study long-term retention, only initial learning and remembering over a short time interval, on the order of just minutes. 
The results suggest that in learning new music, there could be a benefit for performers to use their own, preferred modes of learning first (Korenman et al., 2007). More study on long-term benefits of the original learning mode is needed before significant conclusions can be drawn.

\section{Role of Familiarity with a Piece, Familiarity with a Style, and Likeability}

If a musician is familiar with a piece of music, or familiar with its style, does that affect its memorability? If one enjoys hearing a piece before learning it, will it be easier to learn?

Snyder (2000) explains that schemas provide a structure for memory and improve the chunkability of what one might hear or practice; in this way, schemas aid in memorization. Music of one's culture, and of particular styles or historical periods, provide schematic kinds of expectations for listening. Schemas that one has learned from culture or from musical training provide frameworks of what musical events are likely to happen and in what order, providing one with long-term memories that align with current patterns one may be memorizing. Therefore, schemas provide a structure for memorizing types of music that are familiar (Snyder, 2000, p. 101-102).

In 2013, Stalinski and Schellenberg studied memorability of music that people liked. In their first experiment, participants rated 24 pieces as to how much they liked them. After a short delay period, the pieces were replayed with 24 new, unheard pieces intermixed. Participants determined whether or not a piece they heard was on the original list, and rated how certain they were of this. In this way, researchers were able to study whether participants had better recognition memory for pieces they liked (2013).

In all, six different experiments were performed with many variations, including varying delay times between hearings, the types of ratings that were made, and the amount of confusion imposed by using music snippets that were similar (Stalinski \& Schellenberg, 2013). The research found that higher ratings of liking a piece, and one's ability to recall a piece, were correlated after both a 10-minute delay period and a 24-hour delay period (Stalinski \& Schellenberg, 2013, p. 700).

Stalinski \& Schellenberg (2013) report that in past research, the mere exposure effect (e.g., Zajonc, 2001) has shown that people tend to like something better if they have simply been exposed to it in the past. Therefore, this study sought to determine whether liking influences memorability in return. They succeeded. "The results [of this test] implicate a direct association between liking and recognition. Considered jointly with previous findings, it is now clear that listeners tend to like music that they remember, and to remember music that they like" (Stalinski \& Schellenberg, 2013, p. 700). 
These results may be an underlying factor in why hearing a piece prior to practice aids in its memorization. Also notable are further results of this study that "point to an important link between memory and positively evaluated stimuli" (Stalinski \& Schellenberg, 2013, p. 714).

\section{A Multimodal Approach to Learning and Memory}

It is clear that numerous factors are involved in learning and memorization. The following section surveys an expert, multimodal approach to learning music.

In 1975, Edwin E. Gordon coined the term audiation to represent a complete internalization of music. Gordon's subsequent theory of audiation, expressed in his classic text, Learning Sequences in Music: A Contemporary Music Learning Theory (2012), is based on the way human beings acquire language. Not only do we hear and mimic language while we are very young, but we internalize it and understand its meaning so that we can manipulate language for ourselves. Gordon's process intends to move beyond imitation and rote memorization to incorporate meaning, understanding, and ownership in music learning, starting while a child is very young. As young children learn to use words to create meaningful sentences, children can learn to use musical patterns to create, understand, remember, and enjoy music (Gordon, 2012).

Gordon's educational process begins by teaching small tonal and rhythmic patterns first, as the building blocks of music (Gordon, 2012). However, in Gordon's theory, there are eight types of audiation, all of which may, and should, happen throughout six defined skill levels. Gordon's types of audiation are inherently multimodal. The first three types are provided here with their descriptions.

Type 1. Listening to familiar or unfamiliar music.

The process of hearing and noticing familiar and unfamiliar tonal and rhythmic patterns, from which we hear sequences, expectations, and violations; this provides a level of meaning. Hearing parts that are essential points to the ability to perceive structure.

Type 2. Reading familiar or unfamiliar music.

Hearing notation on a score for music we have or have not heard, and being able to both hear the music and understand its meaning, not only to be able to imitate its contents. This is commonly referred to as "notational audiation," often used by pianists and other musicians in mental practice. 
Type 3. Writing familiar or unfamiliar music from dictation.

The understanding of currently heard music such that one can write it in notation. It is a reverse process from Type 2, but we audiate and then notate what we have comprehended (Gordon, 2012, pp. 27-30).

Audiation types four through eight involve remembering, writing, creating, and improvising, with familiar and unfamiliar music, and the ability to create or improvise in silence (Gordon, 2012, pp. 30-32).

All of these types of audiation occur within Gordon's six levels of audiation. Most importantly, Gordon posits that music cannot be fully learned unless its materials are fully apprehended in all levels by the entire body (2012). Figure 1 shows all six of Gordon's stages of audiation (Gordon, 2012, pp. 3237). In the table, I have suggested a plausible, correlated memory-system level of processing that is akin to each stage. Because this system of music apprehension uses human memory stages, it represents an efficient and comprehensive method of learning.

Figure 1. Gordon's Six Stages of Audiation and Suggested Correlated Memory Processes

\begin{tabular}{|l|l|l|}
\hline \multicolumn{1}{|c|}{ Gordon Audiation Stage } & Audiation Level Description & \multicolumn{1}{|c|}{ Memory Level Equivalent } \\
\hline Stage 1 & Momentary retention & Echoic memory \\
\hline Stage 2 & $\begin{array}{l}\text { Imitating and audiating tonal } \\
\text { patterns and rhythm patterns, and } \\
\text { recognizing and identifying a tonal } \\
\text { center and a tactus }\end{array}$ & $\begin{array}{l}\text { Early processing, Feature extraction, } \\
\text { Feature binding }\end{array}$ \\
\hline Stage 3 & $\begin{array}{l}\text { Establishing an objective or } \\
\text { subjective tonality and meter }\end{array}$ & $\begin{array}{l}\text { STM interacting with LTM that } \\
\text { recognizes tonality and meter from } \\
\text { implicitly developed schemas }\end{array}$ \\
\hline Stage 4 & $\begin{array}{l}\text { Retaining audiation tonal patterns } \\
\text { and rhythm patterns that have been } \\
\text { organized }\end{array}$ & $\begin{array}{l}\text { STM passing information to LTM for } \\
\text { encoding }\end{array}$ \\
\hline Stage 5 & $\begin{array}{l}\text { Recalling tonal patterns and rhythm } \\
\text { patterns organized and audiated in } \\
\text { other music }\end{array}$ & $\begin{array}{l}\text { Activating LTM and context memory } \\
\text { for similar music. Updating and } \\
\text { replacing LTM connections and cues } \\
\text { to other music that is known }\end{array}$ \\
\hline Stage 6 & $\begin{array}{l}\text { Anticipating and predicting tonal } \\
\text { patterns and rhythm patterns }\end{array}$ & $\begin{array}{l}\text { Updating or adding information to } \\
\text { categories and schemas for future use }\end{array}$ \\
\hline
\end{tabular}


According to Gordon, when music is fully understood and internalized, there is no need for an individual step of memorization to happen on its own. Instead, memorization is related to a multimodal approach for music internalization that includes meaning, which facilitates long-term learning (Gordon, 2012). Gordon's eight types of audiation naturally lend themselves to variable practice, which further increases learning, abstraction and transfer. Many of these variable practice techniques are listed on page 66.

\section{Plasticity and Individual Differences in Learning}

\section{Plasticity.}

Plasticity is the ability to create lasting changes in the structure or function of neurons or in brain organization in response to a stimulus, like an experience, or the acquisition of a new skill. (Herholz \& Zatorre, 2012, p. 486). Plasticity allows the brain to "rewire" and reorganize itself in order to change and adapt. Therefore, plasticity allows the brain to learn and is fundamental for memory formation.

\section{Individual Differences.}

According to Guenther in Foundations of Cognitive Psychology: Core Readings, individual differences refer to unique qualities and abilities in each person that may differ from standardized norms (Guenther, in Levitin, 2011, p. 609). Some equate this concept to the Intelligence Quotient (IQ). Further, some believe that the total quantity of overall ability is a single undivided resource that people use for all of their intellectual activity. Currently, research instead suggests that one's abilities are multifaceted and specific to various domains (2011).

While much has been learned about human memorization and learning, each person learns differently. Research has just begun to understand learning differences between individual people. Further, it is known that musicians' brains have demonstrable distinctions from those of non-musicians. This knowledge sheds light on the topic of memorization and also proposes new questions for further research.

Research from approximately 1980 to date has revealed many basic observations about differences in the brains of musicians. In a review about brain plasticity in musicians, Münte et al. (2002) noted that "several brain areas, including the planum temporale, the anterior corpus callosum, the primary hand motor area and the cerebellum, differ in their structure and size between musicians and 
control subjects" (Münte et al., 2002, p. 474). Also, musicians have other anatomical differences in the auditory and motor regions of the brain, such as increased gray matter in right sensory motor regions, the left basal ganglia, and the cerebellum. (See Appendix B.) Musicians who began musical study before age seven were found to have enhanced communication abilities between brain hemispheres, as demonstrated by decreased inhibition in studies of guitarists and pianists. This is likely related to the ability to coordinate movements between both hands (Münte et al., 2002, p. 475).

Another researcher explored musicians' responses to sound. In his 2010 article, "The Musical Brain: Myth and Science," Montinaro found that, in musicians, the brain stem's electrophysiological responses to sound are more pronounced and have less latency than in non-musicians (Montinaro, 2010, p. 444). These brain responses were also correlated to the number of years of music training, perhaps giving musicians an advantage in processing tonal stimuli (Montinaro, 2010, p. 444).

In a variety of studies (Hund-Georgiadis, M. \& von Cramon, D. Y., 1999; Jäncke, L., Shah, N. J., \& Peters, M., 2000; Krings, T. et al., 2000, as cited in Münte et al., 2002, p. 475), musicians were shown to utilize smaller neural networks for motor skill learning than non-musicians, which is presumably due to their ability to make more efficient and controlled movements due to training. The brain is also modified during learning in ways that vary from person to person due to individual differences. Specific scientific research on this topic is currently emerging. The following research represents an excellent example of current knowledge.

A 2018 longitudinal study on cello playing has provided some the earliest scientific information about individual differences and plasticity during music training. Wollman et al. (2018) researched the connections between the auditory and motor systems of the brain and how they might change or expand with practice during acquisition (Wollman et al., 2018, p. E6056). Cello performance in this study provided continuous audio-motor mappings due to the continuous use of the bow. Participants had no musical experience, and were trained only enough to participate in the experiment. They were scanned by $\mathrm{fMRI}$ while playing an $\mathrm{fMRI}$-compatible cello before and after one week and four weeks of training (2018). (See Figure 2.)

The auditory-motor dorsal stream activated very quickly during training, and was activated similarly by performing the learned melodies, or by listening to them after they were learned (Wollman et al., 2018, p. E6064). (See Appendix B.) Because this network was activated no matter the accuracy of a performance, its activation "appears to be a prerequisite of music playing" (Wollman et al., 2018, p. E6064). These results demonstrate clear evidence for plasticity in playing and learning music (2018). 


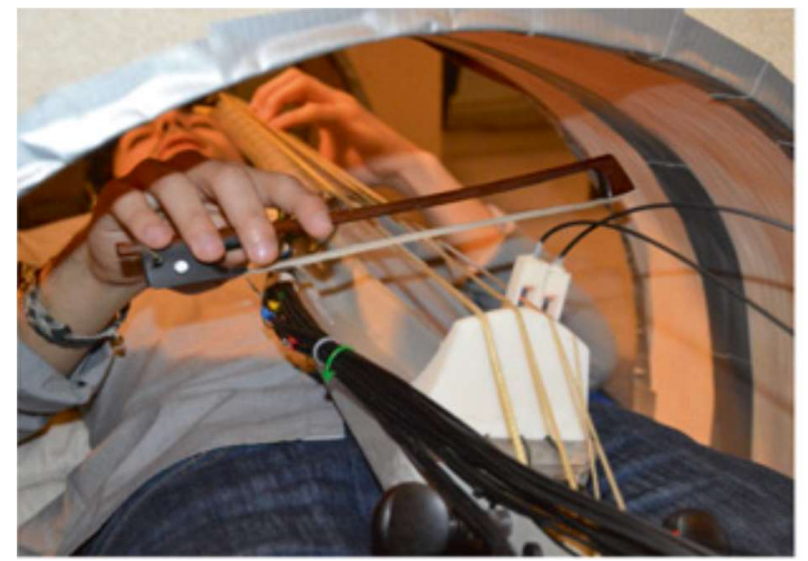

Figure 2. An MRI compatible cello was used for training and scan session. (Wollman et al., 2018).
In this experiment, higher musical proficiency caused more recruitment of auditory encoding areas and motor control areas over a four-week course of training (Wollman et al., 2018, p. E6064). Further, the study showed evidence for individual differences in plasticity because there was stronger activity in areas that relate to coding, specifically in the auditory cortex and hippocampus, in better players (Wollman et al., 2018, p. E6059). Results also revealed a predisposition for cello training, because better

success showed stronger recruitment of the pre-SMA and enhanced pre-SMA connectivity with auditory regions in the better players before the training took place (Wollman et al., 2018, p.E6059). (See Appendix B.)

This important study provides evidence that the dorsal stream is activated by music training, and that the dorsal stream is critical for changes taking place in the connectivity of auditory and motor systems (Wollman et al., 2018, p. E6059). (See Appendix B.) This demonstrates plasticity in music learning. Through greater demonstrated brain activity and connectivity, before and after training, both predisposition for cello playing and individual differences in learning cello performance were confirmed (p. E6059). This study has provided proof of individual differences in music learning, and that brain functions underlie them. The results further demonstrate that brain function is a predictor of musical aptitude (Wollman et al., 2018, p. E6059).

There is further research on plasticity of the brain due to piano training. A 2007 study by Baumann et al. suggests that there is long-term plasticity of audio-motor connections due to intensive piano training. When pianists listen to music, stronger activity occurs in the motor portion of the audiomotor network, in particular, in the pre-SMA, than in non-musicians (Baumann, 2007, p. 73). (See Appendix B.) Non-musicians show a notable amount of multimodal activity, too, but pianists have demonstrated increased motor representation for piano sounds (Baumann, 2007, p. 73).

When musicians or non-musicians listen to music, activity is seen in motor areas, and conversely, when music performance happens, even at a low skill level, auditory areas are active. However, pianists have stronger activity than non-musicians in the areas that overlap the two systems: 
the pre-motor cortex, the SMA, the posterior auditory cortex and the STS (Baumann, 2007). (See Appendix B.)

Methods of acquiring a new skill and the corresponding brain reactions have been studied for quite some time. Acquiring a new skill happens in stages. Performance improves very quickly during early practice while the brain is forming a representation for the new skill (Walker, 2005, as cited in Simmons, 2007, p. 45). This newly formed representation is itself a form of plasticity. After the representation is made, however, performance improvements begin to slow and level off, because brain activation changes as the new skill is continually repeated (2007). The time when slower improvements begin marks a slow-learning phase (Simmons, 2007, p. 46).

When one notices that the rapid improvements during early practice begin to show smaller levels of improvement, one can infer that enough learning and repetition have taken place for a clear mental representation to exist (Walker, 2005). At this point, the new mental representation will be "tagged" for processing and for continued memory consolidation after practice has ended (Walker, 2005, p. 54).

Continued repetition during the slow-learning phase causes an increased area and a larger network of neurons in M1 to represent that skill (Simmons, 2007, p. 53). Therefore, the slow-learning phase is also important to long-term learning. Motor skill networks must be plastic, since they are enlarged as learning takes place.

In 2013, Zatorre wrote an article concerning plasticity related to training versus plasticity related to predetermined individual differences. While it is known that changes occur in the brain relative to practice or auditory-motor skill acquisition, it cannot be proven for certain that these changes are fully due to training (Zatorre, 2013, p. 585). It is possible that preset, physical individual differences, or perhaps other factors, interact with training, causing the changes that scientists observe (Zatorre, 2013, p. 586).

Although a causal relationship cannot be proven, there is significant evidence showing a correlation between the amount of observed changes in the brain and an amount of learning that has taken place (Zatorre, 2013). Similarly, there are correlations between brain changes and the age at which learning a skill began. Finally, longitudinal studies have shown that, in both adults and children, there are clear changes in auditory cortex and brainstem responses after training, indicating that the observed differences were indeed caused by the training (2013). These are all demonstrations of experience-dependent plasticity, and all such evidence taken together strongly suggests that observed brain plasticity is directly related to musical training (Zatorre, 2013). 
Still, the fact remains that the exact state of the nervous system at the time of learning is not identical for everyone (Zatorre, 2013, p. 585). Zatorre notes that, in recent studies, some participants were noticeably faster or slower in learning, and that these states were predictable by observing neural activity, connectivity, or brain structure prior to the experiments. Such was the case in the previously discussed longitudinal study in cello playing (Wollman et al., 2018). In review, Zatorre finds it notable that recent studies "converge in demonstrating that interindividual variability in the distribution of various anatomical features is specifically predictive of subsequent performance or learning success" (Zatorre, 2013, p. 588). However, he also cautions that, "Although much of the evidence seems to deal with the findings in terms of stable traits, given the evidence for experience dependency, it is also reasonable to assume that predispositions may themselves be affected by, or even be the consequence of, ongoing functional and structural changes" (Zatorre, 2013, p. 589).

Many questions remain as to the source of these individual differences. Zatorre (2013) wonders whether they are genetic or environmental in nature. Do the differences relate to the ultimate amount of expertise one can obtain, or to the speed of learning, or both? Are the differences stable over time, or might they change? It is hoped that this line of research will lead to the identification of optimal training paths for each person, paths that are most beneficial to an individual's strengths and weaknesses (Zatorre, 2013, p. 589).

Herholz \& Zatorre (2012) researched whether music training might act as a framework for brain plasticity (2012). Findings concerning specific brain-area changes before and after learning seem to suggest that individual training rates can be related, not only to plasticity, but also to changes in plasticity (Herholz \& Zatorre, 2012). In other words, long-term musical training not only changes the structural and functional properties of the brain, but it also seems to affect the potential for new shortterm learning and plasticity (Herholz \& Zatorre, 2012, p. 493).

Musicians have been shown to be faster to notice regularities and to abstract rules in tone sequences than non-musicians (e.g., Herholz et al., 2009). Research variables also suggest that musicians have a greater potential for motor adaptation to new conditions (e.g., Rosenkranz et al., 2007). Additionally, this research suggests that long-term musical training enhances short-term plasticity of motor skills (Herholz \& Zatorre, 2012). Different rates in training could be seen, visually, in the functional activity of secondary auditory areas of the brain (See Figure 3.) On the whole, it seems that long- and short-term effects of musical training and other types of short-term plasticity interact with each other and may enhance one another (Herholz \& Zatorre, 2012). 


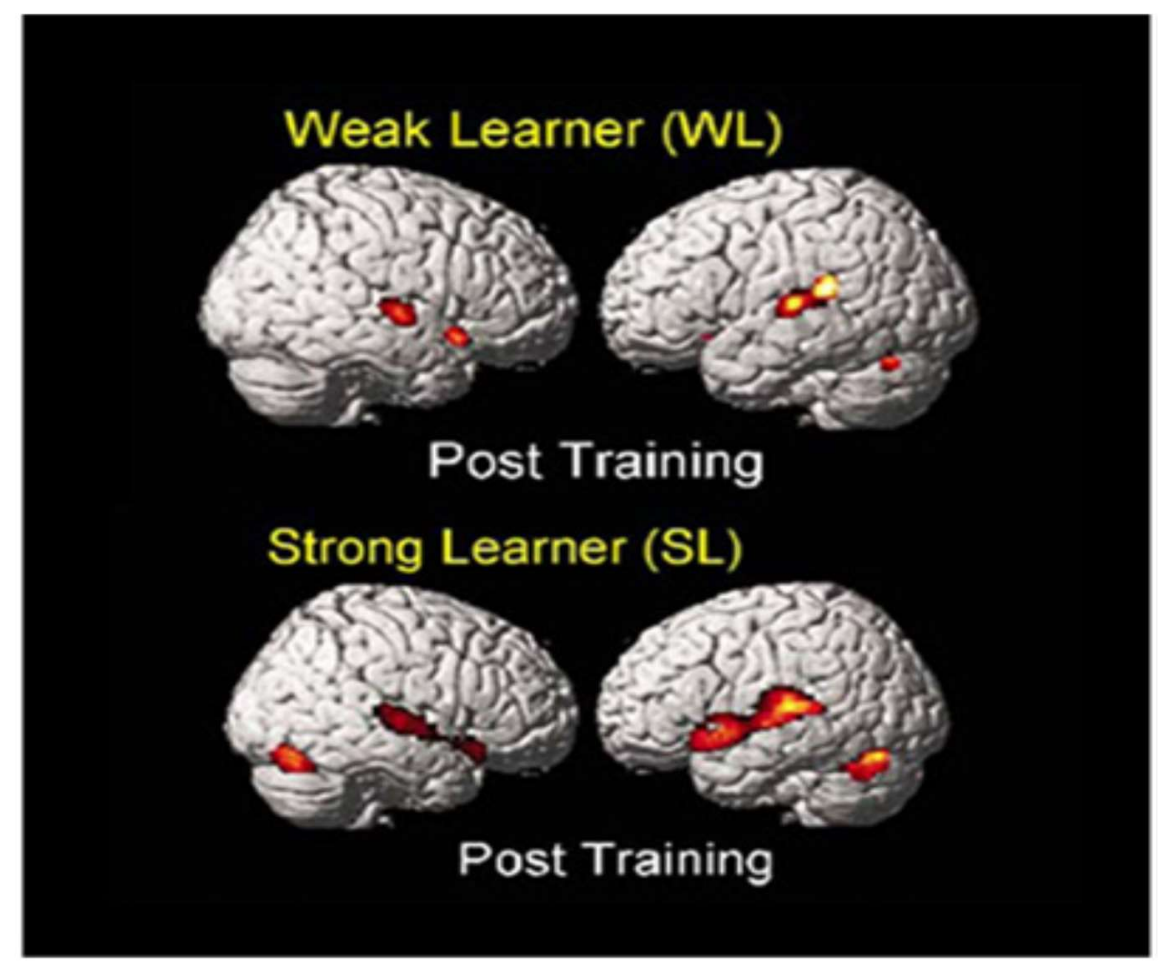

Figure 3. Different rates of behavioral improvement during pitch memory training were accompanied by differential training-related functional changes in secondary auditory areas. These images were captured using fMRI techniques. (Gaab et al., 2006, as cited in Herholz \& Zatorre, 2012)

\section{Memory for Melody}

This section presents background information about how the brain perceives melody, so that, in subsequent sections of this paper, the methods the brain utilizes for organizing melody and for memorizing melody may be presented more clearly.

\section{Neural Correlates.}

Everyone has a musical lexicon, a set of memories of all of the musical phrases to which one has been exposed. In studying memory for musical melodies, Peretz et al. (2009) showed that memory for melody is probably supported by brain structures in the right hemisphere. They found activation in the STS (Superior temporal sulcus) bilaterally, but with a bias toward the right hemisphere. Further, the results suggest that musical memories are "tightly coupled with action or singing" (Peretz et al., 2009, p. 264), because the results demonstrated activation in supplementary motor areas during recall. Interestingly, this research suggests parallels for further study between the workings of auditory streaming and verbal streaming (Peretz et al., 2009, p. 265). 
Prior to this, in 1992, Peretz \& Babai found that the left and right hemispheres process melodic content differently. The left hemisphere processes interval structure, but the right hemisphere uses a contour-based approach to melody recognition (1992). Levitin (2007) comments on these findings, saying that, "in effect [the right hemisphere] draws an outline of a melody and analyzes it for later recognition" (Levitin, 2007, p. 173). Peretz \& Babai contribute that, "contour appears as the best, if not the only, determinant of part structure of melodies" (Peretz \& Babai, 1992, p. 290). They infer that contour has a strong relationship to memory, and they further implicate contour as a potential parsing or chunking device (Peretz \& Babai, 1992, p. 291).

\section{Perceiving Melody.}

Melodic Motion and Expectations. Snyder (2000) explains that the perception of melodic motion is based on building blocks. The minimum size for a melodic grouping is three successive pitches that create two intervals; they are the basic units of a melodic phrase. One interval creates an expectation, and a subsequent interval either confirms, partly confirms, or thwarts the expectation. An interval's function is usually determined by the one that precedes it. If the second interval is the same in both size and direction, the third interval becomes the most important one for expectations (Snyder, 2000, pp. 146-7).

Melodic Schemas. In the same way that tonal hierarchies and intervals are known to be categories, Snyder (2000) presents three specific types of known melodic schemas that can be found across cultures. Axial melodies feature motion around a central pitch. This style of melody is the oldest known way to organize a melody, and is found especially in chant (Snyder, 2000, pp. 154-155). Second, a Melodic Arch melody rises to a climactic high, then falls back down. Generally, it does not use pitches below its centralized pitch. The position of the climax is often closer to the end of the arch than to the middle, and the contour is fairly linear (p. 154). Third, a Gap-Fill melody usually makes an upward leap that is required to be filled. It is most often filled with downward motion, releasing tension. The downward fill is elaborated, making it interesting, but its purpose is to return to the initial tone (p. 155). Melodic schemas provide frameworks for expectations that help in understanding and remembering a new melody (Snyder, 2000, pp. 152-155).

Because one uses schemas for both melody and musical styles, it makes intuitive sense that memory is stronger for melodies of a familiar style than for those of less familiar ones. Several studies have shown this to be true (e.g., Oura \& Hatano, 1988; Zielinska \& Miklaszewski, 1992, as cited in Oura \& Hatano, 2004, p. 309). This section discusses how stylistic and musical knowledge affects melodic memorization. 


\section{Musical knowledge, parsing, and segmentation in melodic memory.}

In 2004, Oura \& Hatano wrote about three types of musical knowledge that contribute to melodic memory, some of which they had discussed in their own prior research. First, order rules are pieces of retained knowledge concerning the form and construction of a musical style (Oura, 1991). These rules guide one's impression of melody as it unfolds.

Second, melodic prototypes are chunks or sequences of several notes that are often used in melodies of a style. These may be structural building blocks, small sequences, or ornamentation (Oura \& Hatano, 2004, p. 309). Using "a rich set of melodic prototypes, a novel melody can be converted into a series of units, each consisting of a prototype and any necessary modifications" (Oura \& Hatano, 2004, p. 309). Melodic prototypes may be necessary for stable memorization. (See the remainder of this section for more information on melodic prototypes.)

Third, pitch names or chord names can be given to constituent pitches for a melody outside a familiar style. Oura \& Hatano (2004) add that, "for unfamiliar and incomprehensible styles, when memorizing an auditory stimulus consisting of a sequence of sounds, labeling strategies of naming notes (Mikumo, 1992, as cited in Oura \& Hatano, 2004, p. 309) and chords (Beal, 1985, as cited in Oura \& Hatano, 2004, p. 309) were used effectively by experienced listeners." These three types of knowledge are all based on schemas.

When extended and realistic melodies are memorized, they must be segmented into units before any further processing can properly take place; otherwise, the number of tones usually exceeds working memory capacity (Oura \& Hatano, 2004, p. 309). Therefore, this research team studied the idea of adding parsing strategies as a fourth component of melodic memorization. They performed three experiments concerning whether participants could determine stable segments when parsing tonal and modal melodies. Afterwards, memory for the melodies was tested (2004).

When parsing the tonal melody, experienced participants segmented the given melody easily into one- or two-measure units, or some combination thereof (Oura \& Hatano, 2004). These participants had years of training playing Western piano music. Inexperienced participants required more trials to accomplish the task, but only a few participants had trouble. With successful parsing, everyone was able to remember the melody (Oura \& Hatano, 2004).

When parsing a Japanese modal melody, the same experienced participants completed the task with difficulty and by using more trials than before (Oura \& Hatano, 2004). Participants commonly reported that they could not hear a beat for use as an endpoint (2004). The inexperienced participants had great difficulty and their segments were deemed scientifically unstable. When they were shown 
how to employ stable parsing, the process still did not help them to remember the melody (Oura \& Hatano, 2004, p. 308).

In this experiment, perfect parsing practices, even when directed, did not guarantee a perfect memory of the melody (Oura \& Hatano, 2004). Full results demonstrated that memorization was stable only when a parsed segment meaningfully matched an LTM melody prototype (Oura \& Hatano, 2004, p. 318).

In any case, lengthy melodies must be segmented for proper memorization. In 2019, Silva et al. determined that the brain likely has a specialized mode for segmenting music. They hypothesized that it does not overlap with how the brain parses speech, and they concluded that top-down processes play an important role in music segmentation (Silva et al., 2019). Pianists are generally aware that memorizing lengthy strings of horizontal material requires a different approach than when memorizing homophonic material. Even if the melody is well-supported by harmonic structure, a lengthy melody must be purposefully segmented for memorization (Silva et al., 2019).

\section{Memorization, Virtuosity, and Expertise in Piano Music}

In 2018, Lisboa et al. set out to describe the relationship between memorization and virtuosity by observing Lisboa, a professional cellist, in 30 hours of preparation of Bach's Sixth Suite for Unaccompanied Cello. The techniques she used were then imparted to a young pianist as a method for learning to memorize while attending to musical goals.

Five aspects of the cellist's practice, as observed by the research team, speak to the relationship between virtuosity and memory. First, practice should always be guided by an image of how the piece should sound (Lisboa et al., 2018). Second, practice must always be guided by goals and the path to obtaining them must continually be evaluated. Third, technically difficult motions must be made automatic. The cellist posits that to gain automaticity, "performers must practice the thoughts and feelings they want to convey to the audience along with the actions that produce the musical sounds" (Lisboa et al., 2018, p. 521). The cellist refers to these thoughts, emotions, and actions as performance cues. Fourth, performance cues help to place focus on the musical thoughts and ideas, thus reducing the chance of memory slips or that a performance might sound over-rehearsed or mechanical. Finally, performance cues act as a safety net, providing places to recover if anything goes wrong (Lisboa et al. 2018, p. 521). 
The young pianist in this study was taught to undertake memorization by documenting her thoughts while she practiced. Her thoughts translated to performance cues, which she used to memorize Schumann's “Der Dichter Spricht," the final piece in Schumann's Kinderszenen (Lisboa et al., 2018).

According to Lisboa et al. (2018), performance cues act as continuing focal points for musical goals, while also reinforcing the cognitive cuing system in moving smoothly from one "thought-motion" to the next. With musical thoughts first and technical thoughts an available second, "a performer learns to play a virtuosic piece with passion and conviction, and from memory. The technical difficulties become invisible and the musical thoughts and feelings make the performance seem magical" (Lisboa et al., 2018, p. 535).

\section{Integrating memory systems in piano performance.}

Chaffin et al. (2016) discuss expertise in piano performing from the perspective of integrating various kinds of memory used in piano performance. Chaffin et al. began by reminding us that spontaneous memorizing during practice happens in the form of associative chains (Chaffin et al., 2016, p. 559). Information in these chains can only be accessed from the beginning, unless purposeful work is done to be able to recall music from other points. They labelled points other than the beginning as content-addressable memory locations (CAML) (2016). According to Chaffin et al. (2016), being able to start at practiced locations throughout a piece is important for practicing structure and for potential recovery from a memory slip.

Preparing CAML is also important because these memories have different properties than associative chains (Chaffin et al., 2016, p. 559). "Content-addressable memories are explicit; it is likely they can be explained in words. Associative chains are more likely to be implicit, and to involve procedural, motor-based knowledge that cannot be expressed in words" (Chaffin et al., 2016, p. 559). This study suggests that pianists should use both kinds of memory together, as seamlessly as possible (2016).

While schemas allow us to store a great deal of information, current theory says that details will be lost when one reconstructs a memory. Therefore, "we would expect performances to be full of mistakes as the musician replaces the exact notes provided by the composer with the musical gist based on generic knowledge of harmonic, melodic, metric, and rhythmic patterns" (Chaffin et al., 2016, p. 561). So, how do pianists play lengthy programs from memory with little or no error?

According to Chaffin, the answer is connected to how memory functions (Chaffin, et al., 2016). Oral traditions, for example, pass music over many generations, using no score, and, for the most part, 
they preserve the structure and content of their music with minimal distortions. The reason for such remarkable results is due to the use of multiple constraints. In poetry, for instance, multiple constraints of rhythm, alliteration, rhyme, and content limit the possibility of memory errors. "Constraints of this sort are found in every oral tradition that has been studied" (Chaffin et al., 2016, p. 561). So it is with piano performance. The rules of musical syntax limit the kinds of mistakes that can reasonably be made.

Yet to err is human. And piano music can be highly complex and challenging. Therefore, purposeful practice that engages all of our memory systems (multimodal preparation) is seemingly the best course to take (Chaffin et al., 2016). Each memory system engaged during performance has its own memory traces. Research is now finding that many such traces are interconnected. Each kind of memory provides retrieval cues and constraints, making music performance much more "robust than memory for text" (Chaffin et al., 2016, p. 561).

Chaffin et al. (2016) explain that auditory memory provides a guiding image of the sound of a piece and also triggers associative cuing. Developing auditory schemas is a form of expertise, because the more melodic, rhythmic, and stylistic patterns one recognizes, the easier it is to have strong expectations (Chaffin et al., 2016). Good aural skills and regular time spent listening to music help musicians to develop schemas, enabling them to perform better from memory (2016).

Because motor memory is procedural, it can only be demonstrated by doing. Even though one can practice mentally, actual playing is one of the ways to feel comfortable knowing that motions are intact (Chaffin et al., 2016, p. 562). In order to fuse mental practice and implicit procedural memory, pianists should select and practice CAMLs until they can be performed fast enough to be of use at the performance tempo (Chaffin et al., 2016). Practice and CAMLs should also be organized around the structure of the music. "Without a clear understanding of the structure, there is no mental map, and no content-addressable memory" (Chaffin et al., 2016, p. 563).

Emotional memory also plays a role in piano memorization, because memories with attached emotion are remembered more deeply (Chaffin et al., 2016). When researchers ask performers to play without expression or emotion; they often do it with great difficulty (e.g., Demos, 2013). Practicing with musical emotion strengthens the overall memory while strengthening the emotional recall cues as well.

According to Chaffin et al. (2016), visual memory has been found to vary from performer to performer. Some pianists claim to see the score the entire time they are playing. Others must watch their hands or choreograph when to look toward a particular place. "Dame Myra Hess described how she could 'see' and 'read' the printed page when playing from memory, whereas Alfred Brendel 
reported that his memory was not visual at all" (Chaffin et al., 2016, p. 563). Performers must experiment to find their own best visual methods (Chaffin et al., 2016).

Linguistic memory is also used by pianists when "reminding themselves what to do" while playing (Chaffin et al., 2016, p. 564). Instructions often point from one modality to another. Chaffin uses examples: "hold back" as pointing to procedural memory or "hear the melody rise over the top" as pointing to auditory memory (Chaffin et al., 2016, p. 564). These types of commands can be rehearsed in working memory in order to affect a plan of action. Internal forms of speech, usually called subvocalization, can aid mental practice or help one to recover if a problem occurs (2016).

Chaffin et al. (2016) provide three principles for expert memory: "meaningful encoding of novel material, use of a well-learned retrieval structure, and extended practice to reduce the time needed for retrieval from long-term memory" (Chaffin et al., 2016, p. 565).

In summary, memorization has traditionally been looked upon as a method to be passed down from teacher to student, as an individualized process one must find for oneself, or as a process of sheer mystery (Chaffin et al., 2016). Chaffin et al. hold that musical memory is not likely to be any more different across individuals than any other human trait, since everyone's neurological system is the same. Important and efficient techniques for memorization and expert piano performance are now openly known, more than ever before, so that everyone may gain from them (Chaffin et al., 2016).

\section{Reward Bolsters Learning}

In 2015, Freedberg et al. studied how reward influences learning. This study sought to minimize the awareness of reward in order to determine if reward improves incidental learning of a stimulus and response, specifically, in motor skill acquisition.

Non-musician participants performed a set of chords as a response to a visual cue. Some chords were rewarded and others not, but the reward system was not explained to the participants in advance (Freedberg et al., 2015). At the end of the test, rewarded chords were performed faster, and connections for the stimulus and response were shown to have also improved. These results are in line with current models of incidental learning that are linked to the basal ganglia and its release of dopamine, a known chemical reaction that influences motivation (Freedberg et al., 2015, p. 15). (See Appendix B.) Results showed that reward can significantly improve learning of stimulus-response tasks (Freedberg et al., 2015, p. 15). 
Reward has also been demonstrated to improve long-term memory of motor skills. In 2019, Hamel et al. performed a test offering monetary rewards either continuously, intermittently, or not at all. This test concerned top-down related reward. The results revealed that those rewarded intermittently or continuously, showed better retention than others, immediately following the learning process (Hamel et al., 2019). However, the continuously rewarded people did not perform better than those who were rewarded only intermittently. This research suggests that intermittent reward may have been enough to add value to the acquisition process (Hamel et al., 2019). Value and meaningfulness during the acquisition process are proposed as possible keys to long-term retention (Hamel et al., 2019).

In his 2012 discussion of brain plasticity, Zatorre reviews literature showing that "it is well known that there are long-term changes to map properties of the auditory cortex as a function of exposure to specific stimuli," [and that changes are] "strongest when the auditory input is behaviorally relevant and if a task is actively trained" (Zatorre, 2012, p. 487). Zatorre concludes that, "cortical remapping and adaptation of neural tuning are critically dependent on the reward value of the learned stimulus" (Zatorre, 2012, p. 487).

\section{Is Music Memory Special?}

\section{The case against popular musical memory theories.}

Memory for music seems to have a mystique surrounding it, likely due to its seeming complexities. For example, many adults can remember complicated physical finger movements of pieces only played in childhood, or lengthy lyrics if they are connected to a melody or a song. Neuro-music research is beginning to explain some of these complexities.

In 2009, Schulkind undertook a study to see if memory for music was special, unique or distinct from other kinds of memory. He researched three commonly held ideas: that people easily remember popular music for a long time, that dementia patients can often play music they had once known, and that music can be an aid in semantic memorization. Seemingly, these abilities would suggest that memory for music had its own special attributes.

In his study, older adults listened to highly popular songs from each year between 1935 and 1994, and were asked to identify the song title, artist, or lyrics that sequentially followed other lyrics from the middle of each song (Schulkind, 2009). If popular songs are well-remembered over long periods of time, a majority of participants would have done well. Instead, responses were generally average at best, and they worsened as the song year became more recent (Schulkind, 2009, pp. 217-218). 
Second, while there is much empirical evidence of dementia patients having unusually clear memories of music, none of the tests performed in this research were in any way conclusive (Schulkind, 2009). Perhaps dementia patients may experience increased arousal, increased attention, or decreased anxiety about remembering that allows them to play songs on the piano (Schulkind, 2009, p. 219), but this research could not conclude anything. However, since special effects in dementia patients do not seem to be revealed with other kinds of stimulation, perhaps memory for music is special in this way (Schulkind, 2009, p. 220).

Third, in the case of music being used as a mnemonic device, it was found that people sing songs used for memorizing rather often, either when they learn the song, or regularly over time (Schulkind, 2009 , p. 221). The special power of music, in this case, was found to be related to the amount of practicing or overlearning involved (Schulkind, 2009, p. 221).

Overall, this study was unable to conclude with any certainty that musical memory is special (Schulkind, 2009, p. 223). However, Schulkind believes that "future investigations will demonstrate that music is remembered differently from other kinds of stimuli because music itself is different from the other kinds of stimuli" (Schulkind, 2009, p. 223). Music conveys complex ideas and emotions using abstractions, received in different ways by different people, that are different upon each hearing. "Given the unique way that music communicates with the listener, it would be very surprising if the way musical material was learned and remembered was not also unique" (Schulkind, 2009, p. 223).

\section{Do special brain areas for musical memory exist?}

In 2009, Groussard et al. researched whether the brain has a separate or special place for semantic memory about music. The results suggest that musical semantic memory is supported by a network in the temporal and prefrontal lobes, bilaterally, and that two distinct networks are at work in these areas (Groussard et al., 2009).

Semantic music knowledge includes all the music we have heard, including well-known tunes that do not have personal, emotionally-charged experiences related to them. These are processed in the left hemisphere (Groussard et al., 2009). In contrast, when a song is remembered as a memory with connections to a personal or emotional experience, episodic memory recalls the song along with the context of when the song was learned or heard, and any personal associations one has with the song. This process occurs mostly in the right hemisphere (Groussard et al., 2009, p. 280).

Further, the simple fact that memory processing for music is now shown to be widespread in the brain may underly the reason that musical memory remains intact in demented or otherwise braindamaged persons (Groussard et al., 2009, p. 280). Networks of the brain that support music perception, 
memory, motor skill, and emotion are located throughout the brain, suggesting that music memory could constitute some of the most complex human processing that exists (Levitin, 2007, p. 85). (See Appendix B.)

Concerning the study of music performance, memory, and brain function, in 1997 Palmer wrote that "interdisciplinary approaches ... are growing, in part because current findings document music performance as a seemingly unique human ability that is not unique in its underlying cognitive mechanisms" (Palmer, 1997, p. 134). In her view, music performance is uniquely a human ability, but its underlying cognitive functions are only special in that they can shed new light on how all human cognition operates.

In 2002, Peretz shared her personal viewpoint that the brain's specialization for music has evolved on a human, biological level, because music has developed in all societies, very early in the process of human evolution (Peretz, 2002, p. 381). Peretz feels that this is likely among the underlying reasons for the development of neural networks that are specialized to process music (Peretz, 2002, p. 381). Peretz might say that memory for music is broadly special to the human race. However, many scientists continue to study birds' memory for birdsong, which is also remarkable (e.g., Bolhuis, J. J., \& Moorman, S., 2015; Moorman, S., Mello, C. V., \& Bolhuis, J. J., 2011; Yu, K., Wood, W. E., \& Theunissen, F. E., 2020)!

\section{Auditory Motor Learning}

\section{Generalized Motor Programs and Motor Schemas}

Schmidt (1975) recounts that in 1971, research about motor skill learning embraced a new concept: the use of memory traces and perceptual traces. A memory trace was first defined by Adams as a "modest motor program responsible for initiating a movement, choosing its initial direction and determining the earliest portions of the movement" (Adams as quoted in Schmidt, 1975, pp. 226). A perceptual trace helped one to recognize whether a limb had arrived at its intended location, using experience and feedback obtained as the motion took place (Schmidt, 1975, p. 227).

Adams' newly proposed theory was the first to address the idea of learning a new motor skill. However, because the brain does not have the capacity to store a motor program for every required movement, the idea of a central control for physical movement became favored. With central control, movements of similar types were thought to be generalized to handle various uses. In 1975, Schmidt proposed properties of generalized motor programs (GMPs) (Schmidt, 1975). 
A generalized motor program (GMP) contains pre-structured commands for a given set of related movements, for example, throwing an object with an overhand motion (Schmidt, 1975, p. 232). When one faces a new, specified task within that set, one observes the specific parameters needed for the GMP to be successful and then employs the program to carry it out (1975).

From this rationale, the idea of a motor schema took shape. A schema for motor skills stores memories of a particular motion one has made and also abstracts rules about that type of movement. A set of these rules comprises the schema, which can be called upon later and be modified for specific situations (Schmidt, 1975, pp. 244-5). For example, the researcher, Bartlett, once explained his skill for playing tennis: "When I make a stroke, I do not . . . produce something absolutely new and I never merely repeat something old" (Bartlett, 1932, as cited in Schmidt, 1975, p. 234).

Four things are stored when a movement is made: Initial conditions, parameters for the current movement, sensory feedback, and an analysis of how successful the outcome was (Schmidt, 1975). After each movement within a motor schema happens, the recall schema becomes more informed about the relationships among its related movements and it becomes more skilled to enable the next instance of that GMP (Schmidt, 1975, p. 246).

One example of evidence for motor schemas is that people can enact motions that they have never made before. Schmidt describes the example of writing one's signature. People are capable of doing this in a small space, when signing a check, or in a large space, on a chalkboard (Merton, 1972, as cited in Schmidt, 1975, p. 244). The signature motion is supervised by a central motor program and applied with different specifications.

In 2007, Simmons summarized modern theory of GMPs.

As a learner begins to practice a new motor skill, a neural representation of that skill, a motor program, is formed in the brain. This motor program represents the fixed relationships between movements that comprise the skill. Generalized motor programs (GMPs) are representations that allow invariantly structured motor programs to be executed under varying performance parameters. Varied practice generates a set of rules, a recall schema, that facilitates the execution of the learned skill in novel contexts while retaining the integrity of the governing GMP. (Simmons, 2007, p. 42)

Simmons (2007) continues explaining that when one varies the practice of a skill, one exercises a recall schema that enables a particular skill to be executed with varying parameters. It keeps the GMP intact, but allows it to be used in specific and new situations. Relationships between movements stay 
the same while different parameters for each component movement of the GMP change to enable the specific action (Simmons, 2007, pp. 42-43).

A pianist must consider the initial conditions for the desired motor movement, as well as the specific musical needs that will tailor the given GMP for the particular situation. Below are listed four constructs for using a GMP as outlined by Schmidt (1975). Using his detailed descriptions and flowcharts, I have created a specific example of how a GMP for piano practice might operate.

\section{An applied GMP for Piano Practice based on (Schmidt, 1975, pp. 235-238).}

1. Proprioceptive information that precedes movement is processed. This might be how fingers are positioned over the piano keys prior to playing.

2. Information about unique parameters is stored. This might be the desire to perform the rhythm of a melody at a particular metronome speed.

3. The pianist evaluates the results after the GMP executes. Was the rhythm played correctly at this speed?

4. Sensory information is processed about how it felt to execute the skill. Did the fingering work well at this tempo, or was it awkward?

Note that this is the process that takes place when a pianist must sight read with only minutes of prior study. It also happens when a pianist studies a score they have never played before; imagining the tempo and motor movements necessary to perform is a way of setting initial conditions and specifying the exact requirements of a GMP.

As pianists interact with a GMP, often referred to as a motor program or motor schema, they store relationships among its structure, its various uses and the observed results. All of this information enhances the recall schema (Simmons, 2007).

Research has shown that stable practice is best for creating a GMP, because the same or predictable methods are used all the time. Stable practice methods use similarities such as practicing in the same tempo, looking always at the same hand, or simply increasing tempi slightly. Stable practice methods enable the initial memory representation to form (Simmons, 2007, p. 42). Conversely, variation in practice is needed for developing recall schemas (Simmons, 2007, p. 43). In variable practice, skills are executed with different parameters each time, or often, placing higher cognitive demands on the pianist which exercise and enhance the abilities of a GMP (Simmons, 2007, p. 43). 
Schemas also seem to support transfer of a skill. Transfer is the use of an already learned skill to perform another that is similar, but new and somehow different (Schmidt, 1975, p. 245). The ability to transfer a skill to similar or to less similar skills is used as a variable in research to demonstrate how a motor skill may be forming or behaving in the brain, or to demonstrate that learning has taken place.

In order to learn complex tasks like piano performance, transfer (even in the simplest sense) is necessary. A child beginning piano lessons generally has no trouble playing in $G$ position (using the $G$ pentachord) for the first time if $\mathrm{C}$ position is reasonably well-mastered. With modifications in parameters, this approach allows easy transfer to all 24 major and minor pentachord positions. Due to variable practice and its interaction with motor programs, an advanced pianist can transfer skills such as balancing voices and creating dynamic contrast, when moving from piece to piece.

In 2003, Meyer \& Palmer studied motor skill and its transfer in order to understand more about timed motor skills in performance. This research suggested that motor movements may be stored as a sequence of fingerings in order, and that their timing information may be stored separately in the brain (Meyer \& Palmer, 2003, p. 248). Since these findings could infer that motor movements, themselves, must be recalled by associative cuing, the results do not seem to fit well with currently accepted models of GMPs. However, if these findings become even partially substantiated, it may be that variety of timing and tempi in practice are required for building a mature GMP that provides the skill and flexibility necessary for successful performance.

\section{Perceptual Learning and Motor Learning}

This section will compare and discuss perceptual learning and sensory learning, versus motor learning, which is learning that happens via physical practice. Primarily, this is a discussion about learning by hearing a piece versus learning by playing a piece.

In 2015, Van Hedger et al. tested the learning of conceptual structure of some melodies versus motor learning of these melodies, directly, by evaluating consolidation of each separate task during wake and sleep periods. The goal was to evaluate the two different modes of learning.

The results of this study showed that conceptual errors were significantly reduced after sleep, but motor errors were not reduced from sleep consolidation (Van Hedger et al., 2015). Further, participants who stated that they spent more time "playing by ear" showed a greater reduction in conceptual errors after sleep (Van Hedger et al., 2015). A second control experiment tested participants after a 12-hour waking period, yielding the same results, demonstrating that original results were not 
tied to the simple passage of time, nor to rhythms of overnight sleep. Therefore, since motor errors were not reduced with sleep, the results "suggest that conceptual and motor learning consolidate differently and that they interact with practice styles" (Van Hedger et al., 2015, p. 163). This study further supports the idea that the original mode of learning may have an effect on the amount and the quality of sleep consolidation (Van Hedger et al., 2015, p. 177).

In 2018, Brown \& Penhune compared the contribution of auditory learning to that of motor learning in motor skill acquisition for experts and novices. They studied the acquisition stage only, by comparing learning-by-hearing with learning-by-playing.

Brown \& Penhune (2018) tested groups of musicians and non-musicians as they listened to a melody, they performed a melody, listened and performed a melody, or saw only a visual cue. Figure 4 shows a participant being monitored by an MRI machine with a piano interface.

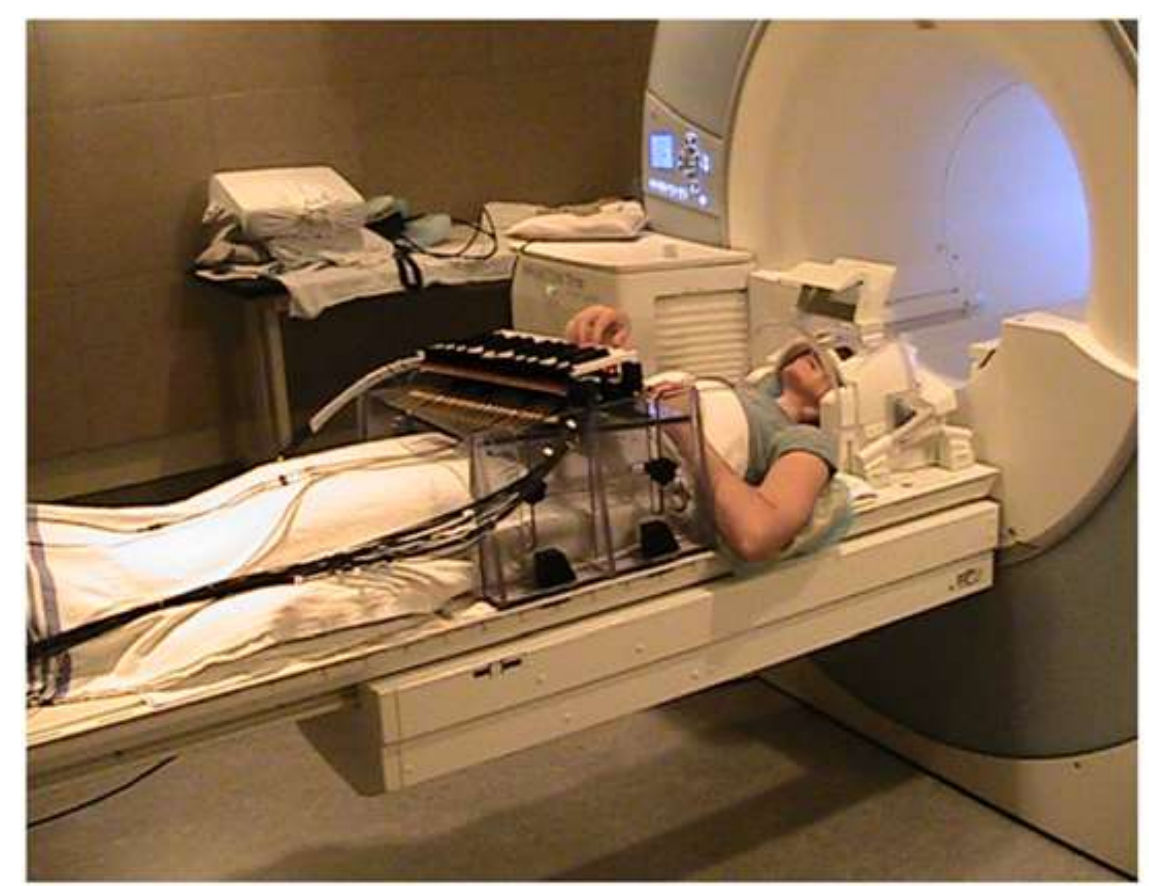

Figure 4. An MRI-compatible piano keyboard in the scanning environment (Brown \& Penhune, 2018, p. 1662).
Results were based on whether pitch (note) accuracy or rhythm accuracy improved in a final performance (Brown and Penhune, 2018). Pianists improved both pitch and rhythm accuracy with all learning methods, but on average, they improved most with auditory learning rather than motor learning (Brown and Penhune, 2018). For non-musicians, pitch and rhythm accuracy also

improved with all learning methods, but rhythm accuracy improved most with auditory learning versus motor learning (Brown \& Penhune, 2018). For both groups, auditory learning was advantageous for rhythm learning, though pianists showed greater rhythm accuracy improvements than non-musicians. Rhythm accuracy improved more in the auditory learning group than all other groups (Brown \& Penhune, 2018). 
Details of the results explain some of the reasons that perceptual learning was advantaged. As would be expected, auditory brain areas were more responsive during listening, and motor areas M1 and S1 were more responsive during motor learning (Brown \& Penhune, 2018). Brain activity increased most consistently in the cerebellum and somatosensory cortex during motor learning (Brown \& Penhune, 2018). (See Appendix B.)

However, with auditory learning, the activities of M1 and S1 were higher during the recall performance, but more importantly, M1 activity during auditory learning predicted improvements in pitch accuracy in the recall performance (Brown \& Penhune, 2018). The STP, STS, and the vPMC network were related to M1's ability to predict improvement. Activity in the IPC also predicted improvement in pitch accuracy (2018). (See Appendix B.)

All the participants, musicians or not, engaged a similar auditory-parietal-premotor network for auditory learning and performance learning (Brown \& Penhune, 2018, p. 1674). Contents of the network included the SMA and PMC, the STG, SPC and IPC, and the cerebellum. The engaged network for pianists also included the frontal lobes, as planning and executive functions were at work (2018). (See Appendix B.)

Overall, pianists should take note that auditory learning was optimal for skilled players. This presented as increased motor cortex activity when performing (Brown \& Penhune, 2018, p. 1674).

Researchers also found that perceptual learning, at any level of expertise, may depend on whether the event to be learned is capable of cuing the motor system well (Brown \& Penhune, 2018, p. 1675). The ability to transform sensory input into motor skills depends on the development of feedforward control networks that cue the motor system. Feedforward control is a method by which the pre-motor and motor cortex prepare to make movements based on sensory input. In this case, the test melodies learned only by listening or viewing a score became sensory input that prepared the motor controls of the body to implement the melody (Brown \& Penhune, 2018).

Musicians with training have better developed feedforward networks than others, suggesting that pianists' ability to engage these networks enabled more efficient learning by listening compared with performing (Brown \& Penhune, 2018, p. 1674). Expertise through experience continues to improve a pianist's feedforward networks, continuing to improve efficiency in motor skill acquisition.

In sum, the results of this study demonstrated an advantage for perceptual learning at acquisition compared with motor learning for both trained and untrained learners (Brown \& Penhune, 2018). 
Prior to this study, Brown \& Palmer (2012) studied how coupling auditory and motor memory affected pianists' memory. They tested skilled pianists when learning new melodies by hearing only, playing only (no auditory feedback), normal practice conditions (coupled auditory and motor skills) and conditions of weak auditory-motor coupling. Motor-only learning was shown to be worst for the pianists' memorization, and importantly, auditory-motor coupling was shown to be better than auditory-only learning (Brown \& Palmer, 2012, p. 567). Pianists who had higher skills in auditory imagery in the experiment outperformed the others in the motor-only learning situation, as their aural skills likely made up for the lack of being able to hear themselves practice (2012). Overall, the findings suggest that motor-learning is an aid to auditory-only learning, but also that motor learning is influenced by individual differences in auditory imagery (Brown \& Palmer, 2012, p. 567).

The longitudinal cello study by Wollman et al. (2018) was also able to compare participants' listening to music vs. performing music while they were being scanned. By comparing listening and playing directly, the study showed that many of the same brain structures in the dorsal stream are similarly activated during both listening and music performance (Wollman et al., 2018). This supports the idea that just listening to music can prime the auditory-motor system to prepare the movements necessary to perform what has been heard.

While conceptual and motor abilities may consolidate and behave differently, the two are yet tied together. "A conceptual error may happen because of a mistake in learning, but when the error occurs, it is also tied to procedural learning, that is, the motor task. Motor errors may well be more influenced by the immediate demands of a specific performance, while conceptual errors are influenced by musical knowledge or musical competence" (Chomsky, 1965, as cited in Van Hedger et al., 2015, p. 176). Chomsky's comments also suggest that various modes of memory interact, so each kind of memory is important for piano performance preparation.

The previously mentioned 2007 study by Baumann et al. was primarily concerned with brain networking that connects the auditory and motor systems, as opposed to comparing the two modalities. Baumann et al. (2007) confirm that "the information flow between sensory processing and motor planning areas is crucial in music performance, which activates an audio-motor network of secondary and higher order structures which are tightly interconnected" (Janata \& Grafton, 2003, as cited in Baumann, 2007, p. 66). Cortical processing is required to transform auditory input into a code that the motor system understands for corresponding output (2007). Both feedforward systems and feedback are required as well. 
No matter the particular auditory-motor advantages musicians may be found to have, the auditory system and motor systems are connected, and experience with piano music appears to strengthen both feedforward systems and network connections in pianists (Baumann, 2007). Overall, a wide range of primary, secondary, and other motor and somatosensory areas were found to be involved in the audio-motor network (Baumann et al, 2007). (See Appendix B.)

\section{Handedness Matters in Motor Learning}

\section{A 2018 study by Smit \& Sadakata researched the effect of handedness on pianists' abilities to} transfer their keyboard skills to different spatial and motor mappings.

It is generally accepted that people understand musical concepts as related to physical space, for example, that pitch height relates to the concept of height in vertical space. Therefore, many people believe that left-handed pianists may benefit from special instruments, namely, a reversed piano where the higher pitches move to the left and lower pitches move to the right (Smit \& Sadakata, 2018). Beginning left-handed pianists have been shown to prefer the reversed keyboard, whereas experienced left-handed pianists prefer a regular keyboard (Smit \& Sadakata, 2018, p. 2). Perhaps left-handed pianists necessarily and regularly create spatial transfers in their minds.

In this study, pianists were asked to change the melodic contour of a phrase or to play a phrase in retrograde so that the fingering had to change (Smit \& Sadakata, 2018). Secondly, they were asked to play on a reversed MIDI keyboard. The length of time required for participants to play the transformed melody, and the number of errors made, were used to determine the quality of the transformations for both left-handed and right-handed, skilled pianists (2018).

The results of this study showed that left-handed pianists were better able to transfer learning than right-handed pianists, especially when the melodies were performed with the left-hand (Smit \& Sadakata, 2018, p. 10). The results also indicated that playing retrograde was more challenging for everyone than using a reversed keyboard (Smit \& Sadakata, 2018, p. 10). Right-handed pianists made significantly more mistakes when they performed retrograde with their left hand than in other transformations, and retrograde was shown to be less difficult for left-handed pianists (Smit \& Sadakata, 2018, p. 10).

Overall, the test results suggest that handedness does have an effect on the way pianists learn a melody, create a mental representation, and apply that knowledge in performance or in transfer (Smit \& Sadakata, 2018). 


\section{Memory Acquisition, Stabilization, Sleep Consolidation}

\section{Definitions and Process}

Memory consolidation is a broadly used term for the process of acquiring a new skill and all of the subsequent brain processes that stabilize, organize or enhance the memory of that skill. The term "memory consolidation" sometimes refers to the component stages of memory development described below. Simmons (2007) describes memory consolidation as the reorganization of new information or the strengthening of new synaptic connections that helps to make temporary, new memory traces into more stable or permanent memories. New memories have a period of instability for a number of hours in which they are easily subject to interference (Allen, 2012). Memory consolidation stabilizes them or gives permanence to them (Simmons, 2007). Simmons explains that the process of memory consolidation begins while a memory is being formed during piano practice and continues in subsequent hours and during overnight sleep (Simmons, 2007, p. 46).

Walker (2005), a forerunner in memory consolidation theory, explains that memory consolidation consists of three large stages. The first stage is acquisition, the process of attaining a procedural skill like piano performance. Acquisition usually requires time periods ranging from several minutes to several hours (Walker, 2005, p. 54). Continued practice beyond several hours may not provide improvement due to fatigue or decreases in attention or motivation, possibly even leading to decreased performance (Walker, 2005, p. 54).

Simmons (2007) explains that during acquisition, a mental representation, or map, of the skill is created in the brain. This is the motor program, the memory, which will undergo modification and improvement under memory consolidation (Simmons, 2007). "A sufficient amount of practice during acquisition is required to trigger consolidation processes; learners must achieve a degree of performance success and complete a sufficient number of repetitions in practice for memories to be encoded and refined" (Simmons, 2007, p. 47). During acquisition, rapid improvement takes place, because brain activity changes while one repeats a new motion and the mental image is formed. "Neurons that fire together during repeated practice of a new motor skill begin to fire together more easily so that existing pathways become readily activated as practice continues" (Walker, 2005, as cited in Simmons, 2007, p. 8). When there is sufficient practice for the formation of the mental representation, improvement starts to level off. When one notices that improvements become 
noticeably smaller, one can assume that the mental representation of the skill has formed clearly enough for consolidation processes to be initiated. The newly formed memory results in the "tagging" of activity-dependent synapses that request plasticity-related proteins which, in turn, become available some hours later (Walker, 2005). This is the chemical process of consolidation at work. The new memory becomes marked for further brain processing after practicing ends (Walker, 2005).

According to Simmons (2007), in the early process of acquisition, newly created neural changes are fragile and subject to interference. If one practices music that is similar to, but not exactly the same as the originally learned music within four to six hours of learning new music, the original memory may be easily disrupted. This may interfere with consolidation of a newly learned skill and result in worse performance, at least in the short term (Simmons, 2007, p. 46).

Walker (2005) explains that if practice takes place too rapidly, the physical changes that normally create a mental representation may not have time to form. In this case, it is believed that already existing neural pathways in the motor cortex can become uninhibited or unmasked, allowing them to fine tune toward the new task (Walker, 2005, p. 54). This is what happens when one is forced to learn and perform something "at the last minute."

The second stage of memory consolidation is consolidation-based stabilization, or simply, stabilization (Simmons, 2007, p. 47). Stabilization allows new information to be retained after a period of time and for new, fragile memories to become less susceptible to interference. Walker describes this as a process of strengthening and maintaining newly formed memories. The ability to execute the new skill becomes dependable, but no additional learning takes place (Walker, 2005, p. 56). During stabilization, "intermittent occurrences of task-related neural activity [take place] that persist beyond practice," strengthening new neural connections for memory of the task (Simmons, 2007, p. 48). Stabilization happens between fifteen minutes and six hours following practice and does not require sleep. It happens during wakefulness or over the simple passage of time (Walker, 2005, p. 56).

Walker names the third stage as consolidation-based enhancement, or simply, enhancement, which requires overnight sleep. Additional learning takes place during this time, without any additional rehearsal, practice, or voluntary concentration (Walker, 2005). This learning enhancement typically provides a modest gain in performance accuracy, speed, or both. The first scientific evidence for sleep consolidation dates back to 1801; it is dependent on chemical process occurring during sleep (Walker, 2005). Neural networks that were active for learning, but not essential afterward, become disengaged, and the same brain activity that happened during acquisition reactivates, "as if the brain is replaying significant events of the day" (Walker \& Stickgold, 2006, as cited in Simmons, 2007, p. 49). 
The first night following practice is crucial for sleep enhancements to take place (Walker, 2005). If the first night of sleep is either inhibited or fully deprived, but sleep happens normally on the second night, a lessened amount of sleep consolidation still occurs (Fischer et al., 2002, as cited in Walker, 2005, p. 57). Some small amounts of consolidation happen on subsequent nights of sleep, as well. However, if one does not sleep the night before learning a new skill, memory formation during acquisition may be impaired (Simmons, 2007, p. 50).

It is interesting that increasing, even doubling, training-session time may not change the amount of sleep-dependent consolidation that happens, nor does the amount of practice have any correlation with the amount of sleep consolidation that takes place. Practicing and sleep consolidation are thought to be controlled by different mechanisms (Walker, 2005, p. 57).

In 2012, Debarnot, Castellani, \& Guillot extended important findings by Kuriyama (2004) by showing that overnight sleep enhancements are related to the difficulty level of the task. Using both one-handed and two-handed finger tapping exercises, they found that the greater the difficulty, the greater the gain. In other words, the brain provides sleep consolidation to tasks of all levels of difficulty, but it provides more sleep-related enhancement for the most difficult tasks one may need to learn. (Debarnot, Castellani, \& Guillot, 2012). Simmons reiterates, "sleep dependent memory consolidation enhances performance of more complex finger skills to an even greater degree than it does less complex finger skills" (Kuriyama et al., 2004, as cited in Simmons, 2007, p. 51; Debarnot, 2012).

Both Simmons (2007) and Walker (2005) discuss details concerning the periods of sleep during which consolidation happens. Rapid Eye Movement (REM) and Non-Rapid Eye Movement (NREM) sleep patterns alternate in humans approximately every 90 minutes at night. Each stage of sleep produces unique neurochemical and electrical patterns that have been observed. NREM sleep has four stages that increase in depth of sleep from stage one to stage four. NREM dominates the first half of the night, especially stages 3 and 4. REM and NREM Stage 2 dominate the second half of the night. Stages 3 and 4 of NREM are often grouped together as Slow-Wave Sleep (SWS), a time when the brain produces synchronous EEG waveforms that are thought to be related to memory enhancement. A positive correlation has been found between memory consolidation for skills and stage-2 NREM sleep, particularly late in the night. Little is known about whether specific sleep stages are specifically required for piano memory consolidation (Simmons, 2007, pp. 49-52; Walker, 2005, pp. 51-53).

In his review article, Walker discusses at length the possible foundations that explain sleep consolidation (Walker, 2005, pp. 60-63). All of them broadly concern the regulation of brain plasticity in either a biological or chemical way. This may involve regulation of chemical neurotransmitters that 
change potentials in neurons for making connections. It may involve oscillations in electrical waveforms like slow oscillations in deep NREM sleep, or low-amplitude wave forms called sleep spindles. Both are believed to be related to memory. Finally, sleep consolidation likely involves molecular and/or cellular processes that produce plasticity (Walker, 2005, pp. 60-63).

Because overnight sleep provides additional learning, daytime napping has also been studied. In 2003, Mednick et al. reported that sleep-dependent learning can take place during a sixty-to- ninetyminute daytime nap if the nap included both slow-wave sleep and REM sleep. Results were found to be similar to those demonstrated in eight hours of overnight sleep. Moreover, observed gains were not replaced or usurped by subsequent overnight sleep-dependent gains. Instead, they added to subsequent sleep consolidation, so that performance in a 24-hour period showed gains that might normally take two full days of time (Mednick et al., 2013).

A recent study by McDevitt et al. studied whether the practice or restriction of regular daytime naps had any effect on sleep-dependent memory. The study included people who regularly napped and those who did not. Surprisingly, the findings showed no changes in either brain activity or performance after four weeks of testing. Results suggest "that individual differences may impact the potential benefits of napping on performance" (McDevitt et al., 2018).

In conclusion, research shows that the brain continues to solidify and enhance initial representations of practice after practice ceases. Stabilization occurs up to six hours past practice and happens with the simple passage of time, not requiring sleep. Sleep consolidation, where learning enhancement occurs without practice, requires one night of overnight sleep, and may continue in a lesser way during subsequent nights. During sleep consolidation, chemical brain processes work on all levels of motor skill enhancement, but provide extra support to enhance the more difficult parts of a task (Simmons, 2007; Walker, 2005).

\section{Review of Musician-Related Research}

Studies concerning piano practice, spacing effects, and memory consolidation have provided a great deal of interesting information. The experiments that follow have varied a number of factors: using inexperienced vs. experienced participants, varying break intervals from minutes to 24-hour breaks with sleep, employing stable practice and variable practice, regulating the number of repetitions or time spent in practice, varying the difficulty of the target material, and intentionally introducing 
potential interference. Many studies to date are based on short, one-handed melodies as target material.

In 2006, Simmons \& Duke performed the first experiment that demonstrated sleep consolidation enhancements for music, using a simple task on a piano keyboard. They demonstrated improvements in performance accuracy with both 12-hour and 24-hour intervals that included sleep, and enhancement of evenness in timing after 24 hours with sleep (Simmons \& Duke, 2006).

In 2007, Simmons was the first to impose regulations on music practice in a study whose participants were inexperienced learners. She found that note accuracy improved with 24-hour breaks that included overnight sleep (Simmons, 2007). Accuracy with higher speeds improved with both 6- and 24-hour breaks, suggesting that speed accuracy improves with either sleep or wake resting periods. Stable practice showed improvements in note accuracy and tempo accuracy over breaks that did not include sleep. Using variable practice, note accuracy improved with 24-hour breaks with sleep, but no break intervals provided tempo accuracy improvement. Simmons postulated that regulating the amount of practice may play a larger role in memory consolidation than had been previously thought (Simmons, 2007).

In 2009, Cash found that five-minute rest periods during the learning of keyboard sequences showed better results when the break was taken early in the practice session rather than later. Retention of the material was worse on the following day with those who took later breaks or took no breaks at all (Cash, 2009). Then, Duke et al. (2009b) again showed that taking five-minute rest breaks early in a practice session provided significantly larger sleep-consolidation benefits than taking a break later in a practice session, or than taking no break at all.

In 2012, Simmons performed a study that was the first to focus on experienced musicians learning a complex skill over multiple, equal practice sessions separated by differently timed gaps. She found that accuracy improved offline only with a 24-hour gap including sleep. Significant increases in speed occurred with participants who had 6- or 24-hour breaks, (waking or sleeping), but speed still increased in all gaps of 5-minutes, 6-hours, or 24-hours (2012). The data indicates that accuracy improves with sleep, and speed improves with both wake and sleep consolidation (Simmons, 2012).

In 2012, Allen studied memory consolidation in musicians that occurs in one night of sleep, and stabilization immediately following practice, in order to explore the extent to which new memory skills may be unstable immediately after learning. This study included potential interference for the first time, by asking some groups of participants to learn more than one similar melody during training. While predictable sleep-consolidation improvements in accuracy and speed did take place in control groups, 
no overnight enhancement happened in performance of the first melody when a second, similar one was learned (Allen, 2012). The results showed, for the first time with musicians, that additional practice on a second, similar melody may inhibit overnight memory consolidation (Allen, 2012). "Of course, since we encounter so many new stimuli in a day, the brain must selectively consolidate those memories that are the most meaningful, important, and potentially beneficial" (Allen, 2012, p. 800). Therefore, the brain likely encountered some conflict as to which material was most important for consolidation.

In 2012, a study by Antony et al. sought information on the age-old idea of learning by osmosis. Can music be learned by simply hearing it while asleep? While the participants took a nap, researchers played a melody that participants were actively learning. Results showed that music skills were strengthened after the music was heard during slow-wave sleep, one particular time when memory processing is believed to occur (Antony et al., 2012). In this study, waves of electrical activity in the right pre-motor cortex, controlling the left hand, were linked to memory processing and the observed performance enhancements (2012).

In the review of this study by Laura Sanders (2012), she claims that the problem with this method is that sleep may not be an optimal time for choosing what skills the brain might sharpen, thereby overriding some of the necessary work of the brain overnight. She quotes Robert Stickgold of Harvard Medical School who commented that, "just because a skill can be sharpened during sleep doesn't necessarily mean that it should be. I think your brain does a lot of triaging at night. Now, you're trying to override that triaging and tell your brain what it should be worrying about" (Sanders, 2012, p. 10).

Allen \& Duke (2013) performed an important study where music practice was restricted, but manipulated such that other kinds of musical-mental competition took place before sleep, while a control group did nothing else prior to sleep. Aside from a learned melody, some participants learned difficult new material on a primary instrument, some reviewed old material on their primary instrument, and some had no other musical activity prior to sleep. The number of repetitions and length of practice time of the target melody were restricted. In this study participants showed significant decrements in performance between evening training and morning test (Allen \& Duke, 2013, p. 67). The decrements varied, but researchers have speculated that restricted, limited practice may interfere with consolidation-based memory enhancement (Allen \& Duke, 2013). In other words, this study supports the idea that "the nature of practice may influence the extent to which consolidation-based gains are obtained" (Allen \& Duke, 2013, p. 72). 
In 2014, Cash et al. tested whether hearing a model performance of a piece prior to practice had any effect on overnight sleep consolidation. The results were significant and positive. Those who heard the model performance prior to practice responded better, both in performance during evening training and in the morning retest after sleep (Cash et al., 2014).

In 2019, Duke et al. sought to substantiate and extend findings from Cash (2009) about early rest breaks in practice by including more complex target melodies and by introducing declarative or procedural memory tasks during the rest breaks. Taking early breaks in practice could easily become an important daily routine for musicians if it improved both short- and long-term gains in retention. This was the first study to examine advanced musicians' learning of a simple motor sequence and also the first to compare advanced musicians and non-musicians directly (2019). However, in this study, Duke et al. found no positive long-term gains from taking early rest breaks, a result which is not in line with Cash's (2009) earlier results (Duke et al., 2019, p. 92). This topic will require more research for clarity. In summary, there are currently some practical results in memory consolidation research. Practicing too much beyond the first acquisition of a motor skill may diminish returns. Instead, taking a break of about four to six hours will likely solidify newly acquired practice memories. Progressing from this point is usually more stable and a more efficient use of time.

Sometimes one must practice when gains are seemingly slow. This kind of practice is still quite worthwhile, because continued practice, even with slow progress, increases the size and strength of the brain's memory representation.

Attempting to learn two new, similar passages at once, may cause an interruption in original encoding of memory. If one is learning a new piece with difficult, similar passages in two different keys, or in similar but varying presentations, it is better to learn them in separate sessions, perhaps with a night of sleep in between.

Learning will usually benefit from taking a nap. Overnight sleep, however, will improve the memories created during practice, and will tend to enhance more difficult skills more than the easier ones. Sleep likely improves note accuracy, but speed accuracy may improve in either waking or sleeping rest periods. Regular overnight sleep prior to practice, and on each night in between, is crucial for memory consolidation processes. 


\section{Piano Practice Techniques and Metacognition in Practice}

\section{Practice Processes Observed in Highly Successful Pianists}

Simmons (2007) cites several case studies of advanced musicians (college music performance majors and professional musicians) that have been conducted between 1989 and 2003, in an effort to identify the mechanisms of practice that are consistently used by the highest achievers (Simmons, 2007, pp. 14-19). In these investigations, musicians were observed as they practiced a new piece in preparation for performance, and their employed strategies were gathered. A basic pattern of common, successful practice is described here, along with supportive research (Simmons, 2007).

First, Simmons recounts research by Chaffin et al., 2003 which "determined that the auditory representation of a piece guides physical practice from the initial reading to performance in concert." This was one of a series of important case studies from 1994-2004 examining the practice of a concert pianist (American-Romanian pianist Gabriela Imreh, also co-author with Chaffin of the book, Practicing Perfection) during her entire preparatory cycle for a public performance (Chaffin et al., 2003, as cited in Simmons, 2007, p. 15). From this study, Simmons further suggests that "advanced musicians' auditory and motor processing work together in initial stages of practice for the proper acquisition of new material" (Simmons, 2007, p. 15). This supports the case for hearing a model performance before practice, then integrating that sound image with physical practice (Simmons, 2007).

\section{Fingering.}

In observations, a great deal of effort in the initial stages of the pianist's practice was directed toward choosing fingering, especially for the most difficult passages. Once selected, fingering patterns remained fixed throughout practice (Miklaszewski, 1989; Chaffin \& Imreh, 2003, as cited in Simmons, 2007, p. 15). In related research, Herholz \& Zatorre (2012) found that changes in the right frontal cortex related to consistent practicing of the same fingerings create important new images in the brain that connect sound to action (Herholz \& Zatorre, 2012, p. 491). Motor areas where the new fingering representations had been created became excited simply by hearing the piece. Practice and encoding seem to be facilitated when fingering remains constant throughout a piece's preparation (Herholz \& Zatorre, 2012).

\section{Structure and interpretation.}

Next, Simmons (2007) reports that pianists shifted their attention to making detailed decisions about interpretation. Participants practiced nuance in phrasing and expressive detail. Notably, these musical goals had already been considered from the beginning (Simmons, 2007). 
Professional musicians use the formal structure of music to organize their practice. This has been shown to produce cognitive encoding that facilitates the memorization and recall of music (Chaffin \& Imreh, 1997, as cited in Simmons, 2007, p. 16). Initial stages of practice are organized around performing the whole piece to solidify musicians' auditory images. This is followed by more detailed work that occurs at the level of major sections, while also working out phrasing and note-to-note issues. This rotational process continues until performance (2007).

Playing through large sections of a piece close to performance tempo early in the learning process helps to solidify one's mental image of a piece, and also helps one to identify where technical and interpretive problems may occur during the learning process (Miklaszewski, 1989, as cited in Simmons, 2007, p. 17). Professionals created goals to organize individual practice sessions, and monitored their practice, using many different practice strategies to accomplish effective learning. According to Simmons (2007), professional pianists:

- listen and compare what they hear to their desired mental image.

- $\quad$ assess problems, and continually assess effectiveness of the strategy being used.

- use a variety of strategies to solve problems.

- use extensive routines of repeating targeted passages to ensure fluid technical execution - all combined with repetition (Simmons, 2007, pp. 17-19).

Advanced musicians choose small chunks of material to isolate during repetitive work (Simmons, 2007), to minimize the time needed for repetitive practice and to allow for focused attention. Short practice chunks are often played at varying tempi during practice to accommodate technical demands. Tempi for repetitions were purposefully selected for the current goal. Difficult component movements were rehearsed hands separately, then together. Altering rhythms was also a commonly employed technique (Simmons, 2007).

\section{Motor Skills, Timing Skills, and Variability in Practice}

In 2018, Caramiaux et al. studied the effects of variation in practice on both motor skill and timing skill in non-musicians. The research utilized different tempi, tempi sets that were small and large in range, and variations on what order tempi were practiced, with the goal of finding associations between variability in practice and performance of motor and timing skills in acquisition. Motor skill level was measured by variability in movements of the participants, and timing skill by the amount of 
variability between keystrokes at the time of test. They studied transfer skills to new and familiar speeds and musical sequences (Caramiaux et al., 2018).

Varying the tempi in a random way was considered to be a form of contextual interference. "Contextual interference is a learning schedule in which a task and its variations are interweaved, and the control variable is the time spent on each task variation. The idea is that injecting contextual interference by frequently alternating motor tasks results in increased long-term learning that is more likely to transfer to new tasks" (Caramiaux et al., 2018, p. 2).

The results showed that practice conditions, stable or variable, had no effect at all on timing skills (Caramiaux et al., 2018). Reducing tempo changes in practice, or using fully stable practice led to better transfer to both new speeds and new sequences (Caramiaux et al., 2018). Higher variability, however, had a detrimental effect on motor skill, which worsened by practicing sets of tempi that were larger in range and in mixed order (Caramiaux et al., 2018). In other words, motor skill transfer was better when the tempo changes were small and remained in order, and variable practice caused interference in motor skill gains. These findings are consistent with the idea that stable practice is better for short-term performance.

The full results of this study indicate that practice variability may be related to expertise of the player (Caramiaux et al., 2018). Therefore, trained musicians may benefit more from higher variability in practice than the less skilled learners involved in this study.

Overall, this test showed that variability in practice had no effect on timing skills, but it negatively affected motor skill at an immediate test (Caramiaux et al., 2018). In early acquisition, then, practice variation affected motor and timing skills differently (Caramiaux et al., 2018). Highly skilled players likely have the most to benefit from varying practice, but for motor skills, variable practice is more beneficial in the long term.

In an important article from 1978, Kerr \& Booth provided compelling evidence that varying the conditions of practice can boost long-term learning of a motor skill. This study involved children throwing a beanbag to a goal located three feet away. Some children practiced throwing only from three feet away. Other children practiced aiming from other locations, two-or four-feet away. In test, the children who varied their practice performed significantly better at throwing the bean bag from three feet away than the children who only practiced from that specific distance (Kerr \& Booth, 1978).

It is now firmly believed that practicing skills similar to, but not exactly the same as the final skill can greatly increase long-term learning in both simple and complex motor skills. The exercises Cortot provides for each Chopin etude are a quintessential example for pianists in varying practice skills for a 
particular outcome. For various works one might be learning, related exercises or transpositions that one may invent are useful for variability in practice.

Indeed, music educators such as Edwin Gordon have suggested strategies for varying practice, including:

- Improvise a melody in the style and key of the passages of your piece (Gordon, 2012, p. 295).

- $\quad$ Sing the melody (Audiation, internalizing the melody).

- $\quad$ Sing or improvise on the bassline (Gordon, 2021).

- Identify the most important tonal melody notes; play only those with the bass (Tonal pattern recognition).

- Identify the underlying rhythm that is easiest to feel. Play while tapping this rhythm (Feeling macrobeats).

- Sing something improvisational, like a countermelody or an extended voice, that does not exist in the music while you are playing (Type 6 audiation) (Gordon, 2021; Gordon 2012).

According to Simmons, 2007, variability in early stages of practice may cause more errors upfront; however, variability involves the memory system at deeper levels than stable practice, thereby improving long-term memory, strengthening recall schemas, and improving subsequent performance (Simmons, 2007, pp. 33-34).

Variability in practice has been shown to work better per the skill and sophistication of the learner, but the difficulty level of the skill to be learned is also a factor. Overall, "learning is optimized when the difficulty of a given task matches the sophistication of learners and when the degree of variability in practice is modified according to that relationship" (Simmons, 2007, p. 6).

According to Simmons, the two strategies which can make a significant and immediate difference in piano practice are distributing practice over time and employing variable practice techniques (Simmons, 2007, p. 10). 


\section{Practice Strategy Research}

\section{Independent learning.}

It has been found that, when learning a new motor skill, movements that the learner produces themself are typically better learned than those that are externally guided or simply observed. "Indeed, research has shown that guiding learners reduces performance errors during acquisition compared with learners attempting to produce the skill without guidance" (Soderstrom \& Bjork, 2015, p. 185). However, early performance errors incurred through self-learning efforts have been found to be beneficial. Guidance has been shown to help in the short-term, but self-learning with more error upfront has been shown to benefit long-term retention (Soderstrom \& Bjork, 2015).

\section{Repetition as stable practice.}

Continuous repetition keeps information in STM for a longer period of time, increasing the probability that it will be remembered in LTM. This theory was researched by Kurtuldu \& Güçlü (2010), using a basic, pre-planned method for repetition of piano music, resulting in a positive outcome in performance.

Two groups of five undergraduate-level pianists were tested using late-intermediate classical pieces they did not know (Kurtuldu \& Güçlü, 2010). Participants were required to repeat two-measure sets, five times each, starting at the beginning. Two-measure sets were expanded to four-measure sets, after which, the next set would overlap by including two prior measures and two subsequent measures. In this way, standard periodic material was interrupted so that practice did not always start at the beginning of a phrase. In the experiment, average practicing included 15 sets of repetitions in 40 minutes of time (2010).

This study also encouraged creative variation in the mind of the participants, for instance, shifting mental focus from one staff to another or one hand to another, in order to avoid boredom, to increase mental focus, and to add some measure of meaningfulness (Kurtuldu \& Güçlü, 2010).

Performance results showed a positive, significant difference for the students who performed the prescribed repetitions vs. those who did not (Kurtuldu \& Güçlü, 2010). This study is an excellent example of stable practice benefitting short-term results. Using this technique would help to ensure that all portions of phrases were being given equal practice attention. (This English summarization was verified by Dr. Kurtuldu in February, 2021). 


\section{Memorization strategies.}

In 2015, Lorenzo et al. studied skill acquisition and overall development in memorizing piano music. Using 121 young adult, student learners under age 35, they sought to explain individual and complex components involved in memorizing motor skills (Lorenzo, 2015, p. 31).

The study found four interesting factors related to perceptions of the participants. These corresponded to whether students believed that memorizing was advantageous, memorizing would improve their interpretive ability, memorizing would likely cause performance problems, or memorizing would help to ensure a completely confident performance (Lorenzo et al., 2015, p. 38).

Coexisting with the players' personal thoughts, the study found five types of strategies for memorization (Lorenzo et al., 2015).

- One creates a mental representation derived from analytical understanding of form.

- One allows implicit memory, and latent memory of associative cues, to be learned without being purposeful. This is learning from just listening or by playing without specific goals or focused attention.

- As one focuses and guides their practice, the required motor gestures and their relation to the sound and emotion they produce become a guide to memorization.

- Using transformation, elaboration, and varied practice, the pianist goes beyond mere repetition. These methods deepen the musical experience, increase the technical difficulty, or add related techniques, including improvising in the style of the piece.

- The pianist must employ metacognition and monitoring of practice by paying attention to the most difficult parts, continually searching for best strategies, continually checking passages melodically, rhythmically, and harmonically. This includes monitoring how memory is solidifying.

(Lorenzo et al., 2015, p. 41).

The research surveyed here supports having a mental representation, allowing for latent memory processes at first, performing skill acquisition, employing variable practice, and using metacognition (Lorenzo et al., 2015).

Soderstrom \& Bjork cite Krueger's 1929 study as demonstrative of the true value of overlearning. In this classic study, half a population of students was asked to overlearn by studying twice as much as a group who mastered the material they were given. After 28 days, the participants in Krueger's study who overlearned the material remembered more than those who did not, and the 
degree of overlearning was related to the degree of long-term retention (Krueger, 1929, as cited in Soderstrom \& Bjork, 2015, p. 178).

Levitin (2011) asserts that overlearning is one of the best ways to achieve automaticity and should not be underrated (p. 349).

\section{Organizational Goals, Practice Structure, and Metacognition}

This section considers research into how learners self-regulate their practice for optimization, including awareness of their own procedures and thought processes.

\section{Knowledge of Results.}

Knowledge of results (KR) is defined as the result of performing a motor action, that is, the result of the performance, not a description of the physical performance choreography (Simmons, 2007, p. 38). KR can be considered as coming from a teacher, or as coming from oneself. Walker (2005) reports that when using stable practice, immediate and constant KR feedback has been shown to be helpful, and that complex tasks benefit from more KR than simple tasks (Walker, 2005).

Simmons (2007) adds that immediate and constant KR feedback has been shown to help during early acquisition, but in more advanced practice, reducing KR is better. Reducing the quantity or frequency of KR helps one to develop independent error detection and self-correction, increasing cognitive effort (Simmons, 2007, p. 40), thereby increasing memorability.

\section{Metacognition, practice organization, self-regulation.}

On organizing practice, Simmons (2007) reports that "organizational structure of practice is more determinative of superior playing in subsequent performance than how much or how long one practices. Professional and advanced musicians engage in goal-oriented, deliberate practice behavior, employing metacognitive skills to monitor their progress over time. In contrast, developing musicians typically do not practice effectively and do not carefully monitor their progress" (Simmons, 2007, p. 29).

\section{Use of best practices and accuracy of self-evaluation.}

Bjork (2018) wrote about his concern that learners are not yet properly aware of fundamental ways to optimize long-term learning. Based on prior research he shared with Richard Schmidt in 1992, Bjork discusses why practice that enhances performance during acquisition is not always the best continued course of action (Bjork, 2018). Bjork explains that it is very easy to be fooled by early practice, because when the ability to perform something improves, it is intuitive to assume that one should 
continue doing the same kind of practice. Instead, new research has demonstrated that procedures that present challenges, and that may slow the rate of early acquisition, are key to long-term retention and performance (Bjork, 2018).

Bjork reiterates that he coined the term desirable difficulties for new practices that cause useful challenges during learning (Bjork, 2018, p. 147). "Contending with them-successfully-can engage the very encoding and retrieval processes that support long-term retention and transfer, as measured by a later test" (Bjork, 2018, p. 147). Desirable difficulties can become undesirable, though, if the learner does not have the skill set to perform them, so Bjork infers that they must fit the individual learner well (Bjork, 1992, p. 147).

A related concept, the testing effect, holds that taking a test on studied material helps to stabilize memory better than restudying (Soderstrom \& Bjork, 2015, p. 186). Practice tests are very strong learning events. For music practicing, the testing effect may be equivalent to a recall effect. It is more useful to practice recalling passages than it is to simply replay and repeat them in their prescribed order. This may include recalling content-addressable memory locations, recalling major sections of a piece in various orders, or recalling pieces in a program in various orders. Recall practice strengthens content addressable memory and stabilizes overall performance memory.

Bjork (2018) reports that there is overwhelming evidence that varying practice conditions, using recall strategies as learning events, distributing practice over time rather than massing practice, and purposefully introducing contextual interference by interleaving skills (See Appendix A), are all desirable difficulties that learners should be embracing (Bjork, 2018).

\section{Judgements of Learning.}

In their article outlining the distinction between learning and performance, Soderstrom and Bjork (2015) discuss findings in new research about what they term "Judgements of Learning." Judgements of Learning (JOL) are self-reported measures of whether learners believe they will remember something at a later time. The authors report that JOLs were shown to be poor predictors of what people can actually recall in the future (Soderstrom \& Bjork, 2015).

Both survey and experimental research in metacognition have revealed that learners often mistakenly conflate short-term performance with long-term learning, ostensibly thinking, "If it's helping me now, it will help me later." The extant survey literature on beliefs about learning suggests that students, by and large, endorse and use strategies that may confer short-term performance gains but do not foster long-term learning. Likewise, research that has examined how people monitor their own ongoing learning has revealed that both retrospective and 
prospective judgments are heavily influenced by acquisition factors, a bias that often produces striking illusions of competence. Given that people act on their subjective experiences, it is imperative that people learn how to learn by becoming knowledgeable of what effective learning entails. It is important, too, that such metacognitive sophistication is fostered early on in one's education. (Soderstrom \& Bjork, 2015, p. 191)

Soderstrom \& Bjork, (2015) explain that there is a critical difference between learning and performance. Learning can happen without noticeable improvement in performance. Conversely, performance can improve noticeably while being accompanied by little or no learning. They contend that research in this area has been so conclusive that it should become common practice for musicians to use it in structuring goals, and for teachers to impart optimal practices to students while they are young (Soderstrom \& Bjork, 2015).

Richard Schmidt, who proposed the original 1975 theory of generalized motor programs, later co-authored an authoritative textbook which emphasizes the difference between performance and learning (Schmidt and Lee, 2011, as cited in Bjork, 2018, p. 148). Schmidt's motor-schema theory provides a basis for understanding why newly supported practice techniques are so important, yet so unintuitive (Bjork, 2018, p. 148). From Schmidt's position, Bjork argues that motor skills being represented in a schematic way in the brain

helps to clarify not only why the notion of muscle memory, which leads to the misguided idea that repetitive practice will stamp skills into our muscles, is so wrong, but also why manipulations such as interleaving, variation, and reducing feedback during training can enhance long-term retention and transfer of to-be-learned skills. (Bjork, 2018, p. 148) Finally, Soderstrom \& Bjork (2015) discuss their theory of storage strength vs. retrieval strength. "Storage strength refers to the degree to which memory representations (i.e., knowledge and procedures) are integrated or entrenched with other memory representations, whereas retrieval strength represents the current ease of access or activation of those memory representations given current mental and environmental cues" (Soderstrom \& Bjork, 2015, p. 191). Retrieval strength is an index of how current performance will be, while storage strength is an index of long-term learning (2015).

The authors posit that long-term memory does not decay or eventually disappear if it is not used (Soderstrom \& Bjork, 2015). Instead, they suggest that one's current retrieval strength works inversely with storage strength. Material that is currently very easy to retrieve is helpful to short-term performance, but lessens the ability for one to build long-term strength. Therefore, creating situations 
that produce "forgetting" is optimal for learning (Soderstrom \& Bjork, 2015, p. 192). Of course, these situations include distributed practice, recall strategies, variability, and interleaving during practice. Forgetting-and-recalling builds storage strength, long-term retention, and transfer skills, all key to optimal practice and performance.

\section{Learning music performance over the long term.}

In 1997, Palmer \& Drake studied monitoring and planning in the large-scale acquisition of music performance skills. Their research found that planning and monitoring skills increased most during the acquisition phase of large-scale learning, that is, during the first three to five years of music study (Palmer \& Drake, 1997, p. 381). In other words, skills for self-monitoring increase most between beginning and intermediate stages of long-term learning. Further, sensitivity to musical structure increased in all skill levels from acquisition to skilled artistry (Palmer \& Drake, 1997, p. 381). At advanced stages, then, pianists should continue to self-monitor, but also be aware that increased sensitivity to one's expressive choices is more likely to provide progress toward artistry. This research further supports that excellent practice and monitoring techniques should be learned very early in the course of becoming a musician.

\section{Best Practices of Advanced Musicians}

A study of 17 advanced pianists by Duke, Davis, \& Simmons (2004), as cited in Simmons (2007), reported observations of the pianists in an unrestricted, single session of practice on a passage of music. This research identified 11 items common to the most successful performers:

1. Playing is hands-together early in practice.

2. Playing is with inflection early on; the initial conceptualization of the music is with inflection.

3. Practice is thoughtful, as evidenced by silent pauses while looking at the music, singing/humming, making notes on the page, or expressing verbal "ah-ha"s.

4. Errors are preempted by stopping in anticipation of mistakes.

5. Errors are addressed immediately when they appear.

6. The precise location and source of each error is accurately identified, rehearsed, and corrected.

7. Tempo of individual performance trials is systematically varied; logically understandable changes in tempo occur between trials (slow down enough; do not speed up too much). 
8. Target passages are repeated until the error is corrected and the passage is stabilized, as evidenced by the error's absence in subsequent trials.

9. When tempo is changed, the first trial at the new tempo is accurate.

10. After the initial learning, phase errors are intermittent (no persistent errors).

11. At least $20 \%$ of all starts are complete, correct performances, though not necessarily at the target tempo.

(Note that this study utilized a single passage, not an entire piece.) (Simmons, 2007, pp. 20-21).

Simmons also observes, "The fact that the best performing pianists took no less time to learn the passage than the other pianists is also notable, because it contravenes the notion that the pianists who performed best on the retention test were more highly skilled than the other pianists and thus were able to learn the passage more easily than the others" (Simmons, 2007, p. 20).

In 2009a Duke et al. reported on the 17 pianists above, finding that the best performers used all of the following three traits in concert:

1. The precise location and source of each error was identified accurately, rehearsed, and corrected.

2. Tempo of individual performance trials was varied systematically; logically understandable changes in tempo occurred between trials (slowed down enough; didn't speed up too much).

3. Target passages were repeated until the error was corrected and the passage was stabilized, as evidenced by the error's absence in subsequent trials (Duke et al., 2009a, p. 318).

\section{Breathing and Memory}

Pianists commonly know that music breathes; however, they often forget to breathe physically with their performances. Alternatively, they may underestimate the role of breathing in practice and performance. The following research suggests that breathing cycles may be deeply involved with and, perhaps, necessary for music performance and memorization.

Recently, "respiratory phase has been shown to influence higher-frequency oscillations associated with cognitive functions, including attention and memory" (Heck et al., 2019, p. 563). In 2019, Heck et al. reviewed recent research and concluded that respiration may play a key role in organizing cortical activity in memory processes (Heck et al., 2019, p. 563). 
The olfactory bulb receives direct sensory information from the nose and interacts, in both directions, with many other cortical areas (Heck et al., 2019, p. 564). Oscillations of neurons interact with the organization of excitatory and inhibitory neurons, producing different types of oscillations, each classified per their frequency. Three of these kinds of oscillations are used by the hippocampus and prefrontal areas during memory processing, and all three of them have been shown to be modulated by respiration (Heck et al., 2019, pp. 566-567).

Freeman \& Rogers found similar cortical activity patterns associated with learning and memory in the visual, auditory, and primary somatosensory areas (Freeman \& Rogers, 2002, as cited in Heck et al., 2019, p. 564). The detailed patterns found in all these modalities suggest that "respiration-locked rhythms could be of more general importance and are possibly present throughout the cerebral cortex" (Heck et al., 2019, pp. 564-565).

Memory was found to be more reliable during inspiration, but only if subjects were breathing through the nose (Zelano et al., 2016). No effects were found when participants breathed through the mouth. Nakamura (2018) found that recall of information was delayed if it had been presented during the transition from expiration to inspiration, suggesting that the breathing cycle affects memory (Nakamura et al., 2018). Overall, "substantial evidence strongly links respiration to major neuronal activity patterns associated with memory" (Heck et al., 2019, p. 567).

Because the structure of music underlies music memory, it seems likely that breathing in concert with structure may someday be shown to have a relationship to memorizing piano music.

\section{Working Memory for Music}

\section{The Phonological Loop and the Tonal Loop}

Schulze \& Koelsch (2012) describe the phonological loop as a process of working memory (WM) that handles auditory and verbal information. It consists of a storage component called the phonological store, where one keeps STM information for rehearsal, and a mechanism that maintains memory, strengthens it, and sends it to LTM if sufficient rehearsal warrants this. The phonological store acts like an inner ear that receives and stores verbal and audio information. The brain converts this information into speech that can be rehearsed. The mechanism component is used to articulate and repeat the information, which circles through working memory like a loop. Repetition may be aloud, or under one's breath, overt, or covert. The auditory part of WM stores words and phonemes, pitch, speech prosody, 
and timbre; the latter three are referred to as tonal WM. Tonal WM manages the maintenance and the active manipulation of tonal information (Schulze \& Koelsch, 2012, pp. 229-230). This description adds to the concept of rehearsing contents of STM, so that it remains active beyond the STM time window and may become persistent for LTM encoding. (See pp. 12-23 for more discussion about STM rehearsal, working memory, STM, and LTM.)

In 2012, Schulze \& Koelsch researched whether music uses its own phonological loop for tonal working memory. They found that in both tonal rehearsal and verbal rehearsal, the activated networks were virtually identical in non-musicians (Schulze \& Koelsch, 2012). However, in musicians there were observed differences between tonal and verbal rehearsal in a number of network structures, demonstrating the existence of a phonological loop to maintain verbal information, and a second, tonal loop for maintaining tonal information in musicians (Schulze \& Koelsch, 2012). Perhaps future research will confirm this as the reason musicians are advantaged with working memory efficiency. It suggests that musicians may have as much as double the working memory capacity of non-musicians, if both general and musical contents are taken into account. (See pp. 10-23 for more discussion about STM rehearsal, working memory, STM, and LTM.)

\section{Working Memory Capacity and Chunking}

In 2018, Ding et al. performed a behavioral study on tonal working memory (WM) in musicians and non-musicians. This study investigated to what degree the number and the duration of notes in a sequence influence tonal WM (2018).

Results show that the number of notes, but not the duration of notes in a tone sequence, significantly affects tonal WM performance for both musicians and non-musicians (Ding et al., 2018). In the study, musicians outperformed non-musicians in a manipulation task, where participants were asked to determine if the last note they heard matched a note of some distance, $N$, prior to it (2018). However, both musicians and non-musicians performed equally well when matching tones while thinking in a forward direction. The summary findings indicate that the capacity of tonal WM is determined by the number of notes, but not the duration of notes in a sequence to be memorized (Ding et al., 2018). These results also support the existence of a separate mechanism for tonal WM. This description furthers the concept of how working memory operates with tonal material in both musicians and non-musicians. Because musicians have been shown to have better working memory operation, overall, more research is warranted to clarify how tonal and non-tonal working memory contents are 
treated in people with and without musical training. (See pp. 10-17 for more discussion about STM rehearsal, working memory, STM, and LTM.)

In 2009, Akiva-Kabiri et al. were the first group to show that maintenance of information in tonal working memory is affected by both the number of items it contains and the rate of presentation, and further, that these two factors interact with each other. Longer sequences of notes were better recognized if they were presented faster, but a fast presentation rate did not improve recognition of short sequences (Akiva-Kabiri, et al. 2009). They posited that long sequences of tones required segmentation and chunking, which reduced working memory load. (See pp. 10-17 for more information on STM, chunking, and working memory.) This supports chunking as a method for increasing working memory capacity, and that long melodies must be segmented by parsing. On the other hand, short sequences were remembered better element-by-element. Without a need for grouping, a faster presentation rate did not provide any advantage, and the slower presentation rate may also have allowed more time for covert rehearsal of each separate element (Akiva-Kabiri et al., 2009). This new research may shed light on how a pianist may sing or listen to long and short melodies in practice and for segmentation.

In 2017, Talamini et al. performed 29 studies that included 53 memory tasks in order to test the ability of LTM, STM, and WM in musicians and non-musicians. Musicians outperformed non-musicians in LTM tasks. However, musicians showed a large advantage in STM and WM for tonal input. Musicians were shown to have better STM and WM capabilities than non-musicians, especially for music (Talamini et al., 2017).

In 2019, Jain \& Nataraja studied fatigue in musicians. Because musicians are prone to fatigue, they posited that fatigue may limit the enhancements musicians have with working memory. The research determined that musicians should take measures to reduce fatigue, such as interspersing short naps between practice sessions, and distributing practice (Jain \& Nataraja, 2019). This study further suggests that fatigue is an important enough factor to always be considered as a variable in future research about cognitive processing in musicians (Jain \& Nataraja, 2019). (See pp. 57-74 for a more complete discussion about napping, distributing practice, and practice techniques.)

In 2014, Grimault et al. demonstrated that brain activity increased with the number of items held in auditory STM during the retention interval of an auditory memory task. (Auditory STM in this study is essentially the same as tonal WM, as the terminology in research is often used interchangeably.) Research showed that the measurements varied per participant, demonstrating that individual differences were a factor in this experiment (Grimault et al., 2014). Further, they also found that the 
frontal and temporal cortices played a role in the retention of pitch information. In sum, they demonstrated that there is a strong connection between the frontal and temporal cortices and the function of auditory STM, and that individual differences interact with these operations (Grimault et al., 2014).

\section{Working Memory in Jazz and Classical Musicians}

In 2018, Nichols et al. studied working memory in jazz and classical musicians, using college students and professionals from each background, in order to see if different styles of musical training had any effect on working memory function. Overall, both groups performed equally well on working memory tests. These included tests for auditory skills, memory in dictation, replaying a watched piano performance, and notating a watched piano performance. Results showed that jazz musicians generally did not have better working memories than classical musicians (Nichols et al., 2018). But surprisingly, "classical musicians did not show superior visual memory for notated pitches, despite their general score-based approach to music performance" (Nichols et al., 2018, p. 104).

Not surprisingly, however, they found that jazz musicians excelled at hearing a set of notes and reproducing them on the piano (Nichols et al., 2018, p. 105). Also, working memory span for this test was positively related to years of jazz ensemble experience. The jazz players were skilled at listening to sound patterns, maintaining them in working memory, and translating the memory into the motor skill needed to produce it. As a conclusion, it is suggested that further research into musicians' cognitive skills should consider the type of training a musician has had (Nichols et al., 2018, p. 101).

\section{Different Attention Mechanisms Available to Working Memory}

In 2018, Katus \& Eimer demonstrated that there are different attention mechanisms available to working memory for different modalities, thus allowing working memory to have some characteristics of parallel processing. In particular, this research studied the behavior of visual and tactile working memory representations. Research found evidence that new mental representations based on incoming stimuli are stored in the same cortical area that encoded them, and that each modality has a control mechanism that can be activated independently or in parallel by multi-sensory working memory, including verbal working memory, tonal working memory, etc. (Katus \& Eimer, 2018, p. 644). 
The results show that tactile and visual WM are supported independently for piano practice and performance (Katus \& Eimer, 2018). As examples, visual attention to a score does not impede tactile input, and looking only at the hands does not impair sensory feedback from the fingertips. Purposeful practice attending to one or multiple modalities at once is likely to be helpful, without concern for overload.

\section{Attention and Awareness}

\section{Attention and Awareness in Practice}

Attention is the process that selects what will be processed in working memory and what becomes prioritized in the FOA. According to Münte et al. (2002), attention to sensory input has been shown to underly experience-based plasticity or learning (Münte et al., 2002, p. 473). WM works together with attention to maintain information after attentional processes have selected the information to be processed. Attention has been shown to optimize working memory in a goal-oriented fashion, by using a set of processes that allow working memory contents to be prioritized and prepared for action (Myers, Stokes, \& Nobre, 2017). (This will be discussed further on pp. 100-101.)

In 2019, Emily Allen et al. studied whether paying attention to one musical feature over another had any effect. Researchers looked for separation of the cortical memory representations, spatially, in their corresponding cortices, in order to determine whether attention to one feature over another created any enhancements in brain representations. They used single auditory events for the study, with pitch and timbre as the two features to attend. Timbre was varied only by one feature they labelled as brightness, so that changes in timbre could be clearly heard (Allen et al., 2019).

The cortical regions underlying both pitch and timbre representations overlapped, but results showed that attention to one feature over the other demonstrated "distinguishable activation patterns even in the absence of physical differences in the stimuli" (Allen et al., 2019, p. 3292). In other words, they found no physical separation in the two mental representations, but the brain did activate distinct patterns when participants paid attention to different features, even when there was no particular reason to do so. Brain representations were shown to be distinct for stimuli receiving attention (Allen et al., 2019).

Is attention effective in the absence of awareness? In 2016, Webb et al. used a masking technique to create states of awareness and non-awareness in participants while attention was drawn to a stimulus, to determine the effects of attention without awareness. In both cases, the stimulus was 
attended; however, when awareness was missing, neural factors relating to attention changed significantly. These included a reduction in the stability of awareness over time and a lessened ability to suppress unimportant stimuli (Webb et al., 2016). Like motor schemas for movement, the brain also has an attention schema that controls attention; this attention schema suffers without awareness (Webb et al., 2016, p. 842). This study was based on visual tests, but it is likely that future findings for auditory stimuli may be similar. For now, this study may suggest that pianists should create goals for practice, while remaining aware of what is being practiced. Otherwise, attention is likely to suffer, reducing one's ability to create clear, strong mental representations.

\section{Attention and Flexibility}

How does one remember important material if attention is divided or distracted? In 2017, Middlebrooks et al. investigated this question using participants who studied with background sound, familiar or novel, or with different kinds of interruptions. The results may easily generalize to practicing with background noise from nearby practice rooms, or with general interruptions. Results of the study showed that value or importance placed on items to be remembered always outweighed either background noise or distractions, even if background noise was unfamiliar (Middlebrooks et al., 2017). In other words, goal-oriented attention overcame adversities in these study situations. "Participants compensated for limitations owing to divided attention by devoting their remaining resources to the most important items" (Middlebrooks et al., 2017, p. 1114). Goal-oriented practicing likely behaves similarly.

In 2016, Wöllner \& Halpern researched attentional flexibility in pianists and conductors, that is, the ability to switch from one attentional stimulus to another quickly and to evaluate when it is optimal or desirable to do so. Some overall findings of this research are of interest. They found that larger WM capacity provides better selective attention, but it also predicts better divided attention (Wöllner \& Halpern, 2016). In this study, conductors showed higher attentional flexibility than pianists; however, musicians are advantaged overall in having excellent flexibility and working memory skills, compared to non-musicians (Wöllner \& Halpern, 2016). 


\section{Emotion Affects the Focus of Awareness}

There is some emerging evidence that the mood one is in during practicing or performing has an effect on attention. This could mean that relatively deep states of happiness or sadness might detract from practice gains, or that one might beware of too much jovial behavior backstage just prior to a performance. The following findings are of interest.

Pacheco et al. (2016) demonstrated that the emotional state of sadness detracts from attention. In 2016, the same team set out to determine if the state of happiness produces a similar effect. Results showed that happiness aided distraction; however, later in the process of concentration, happiness could help one to recover attention again (Pacheco et al., 2016). From this research, they posited that happiness and sadness may both decrease one's ability to filter out unimportant stimuli. This may be due to emotions tapping into mental energy that would otherwise be used to control attention, or due to extraneous thoughts that invade one's mind because of an emotional state. In all observations from this study, they "speculate that happiness might interfere with the disengagement of attention from a deviant sound back towards the target stimulus ... but help subsequent cognitive control to recover from distraction" (Pacheco et al., 2016, p. 419).

\section{Selective Attention Works with One's Knowledge Base and with Working Memory}

In 2018, Wu et al. produced a paper concerning attention in learning or practicing, along with one's ability to select it or to be taught how to select it. They write that there is a connection shown from past research in the relationship between attentional selection and prior knowledge gained from learning and experience. "[An] influential focus of research has investigated how information held in memory guides the orienting of attention. A wealth of evidence points to the influence of the contents of memory on attentional selection" (Wu et al., 2018, p. 1750). This study supports the concept that LTM acts as a filter for allowing contents into working memory and that expertise is the best predictor for success in a field. This study also suggests that current knowledge could be better utilized for people to learn how to optimize selective attention skills (Wu et al., 2018).

In studying the overlap between selective attention and working memory, Gazzaley \& Nobre (2012) demonstrated that top-down processes modulate selective attention and working memory, because prefrontal and parietal regions were shown to be active while a stimulus occurred, while expecting a new stimulus, while selecting and encoding stimuli, in maintenance tasks, and in memory 
retrieval (Gazzaley \& Nobre, 2012, p. 129). Top-down modulation underlies our ability to focus attention on task-relevant stimuli and ignore irrelevant distractions (Gazzaley \& Nobre, 2012). This simply underscores that giving attention to one's goals is an important, active choice.

It is increasingly evident that attention is also crucial for prioritizing information in WM as the importance of its individual items changes in real time. In 2017, Myers, Stokes, \& Nobre suggested a modern model of how WM prioritization may operate. They posit that prioritization may act in multiple steps. First, attention works toward selecting a memory. Next, it reconfigures a memory's representational state so that incoming demands can be handled. The process of reconfiguration sets up a perception-action mapping, so that attention on one item does not have to be sustained and actions may be prepared (Myers, Stokes, \& Nobre, 2017, p. 449). Interestingly, this theory links the processes of working memory with task switching and with preparation for actions (Myers, Stokes, \& Nobre, 2017), both of which are highly related to piano performance from memory. 


\section{Memory and Cognition in Performance}

\section{Auditory Imagery: Definitions and Discussion}

Auditory imagery (AI) is assumed to be a multimodal process where the mind imagines auditory features of sound that are not present in the real world (Keller, 2012). Auditory imagery may be generated automatically, but it is generated deliberately by performing musicians. Simmons describes it as any "mental representation of repertoire" (Simmons, 2007, p. 14). For performing pianists, then, auditory imagery is any voluntarily or deliberately created imagination or representation of repertoire in one's mind. It has been demonstrated to be important for successful piano performance from memory (Highben \& Palmer, 2004, p. 58).

In 2015, Saintilan described auditory imagery as being "unique to the individual" and used by musicians to structure their performing (Santilan, 2015, p. 309). For example, Horowitz is known to have practiced mentally before playing in concerts and Rubinstein did so in order to facilitate daily practice (Meister et al., 2004, p. 219).

Some definitions of Al make allowances for involuntary auditory imagery. People experience involuntary imagery when a song becomes "stuck in one's head." These "earworms" were referred to in 1992 by Intons-Peterson as: "the introspective persistence of an auditory experience, including one constructed from components drawn from long-term memory in the absence of direct sensory instigation of that experience" (Intons-Peterson, 1992, as cited in Santilan, 2015, p. 46).

While everyone may experience earworms, everyone may also experience voluntary or involuntary imagery. In 2019, Kussner et al., reported that $77 \%$ of people they studied have experienced visual imagery while listening to music, whether or not they had musical training (Kussner et al., 2019, p. 59). Herholz et al. (2012) adds that, "even if one is not a trained musician, it is possible to come up with a mental auditory image that resembles the real experience of hearing that song even before it starts playing. Auditory imagery is an aspect of auditory cognition in which auditory information is internally generated and processed in the absence of real sound perception. It can be surprisingly accurate, reinstating many aspects of the real stimulus in the mind" (Herholz et al., 2012, p. 1382).

In 2012, Halpern writes that "the experience of hearing something in one's head is a phenomenologically strong one, particularly for music, and is accompanied by identifiable changes in cerebral blood flow as well as neural electrical signals" (Halpern, 2012, p. 37). Keller (2012) notes that imaging techniques now allow researchers to observe if a musician is using imagery and to see what 
happens in the brain while one imagines specific pieces of music over the course of time (Schubotz, R.I., 2007; Zatorre, R.J., J.L. Chen \& V.B. Penhune, 2007, as cited in Keller 2012, p. 207).

Zatorre (2012) writes that many studies have converged in showing that auditory imagery benefits musicians. Zatorre claims that, "there is in fact good evidence that mental practice-which no doubt includes more than just an auditory imagery component-can be beneficial to musicians and can even result in changes in cortical functional organization. These studies confirm the utility (and hence the psychological reality) of musical imagery for musicians and performers" (Zatorre, 2012, p. 222). In a related study, Pascual-Leone (2003) also showed that mental practice improves performance (PascualLeone, 2003, as cited in Zatorre \& Halpern, 2005, p. 11). More evidence has shown that motor cortex representations grow with imagined practice in the same way that they grow with real practice (Zatorre \& Halpern, 2005, p. 11).

It is now generally understood that there are many kinds of imagery. These include audiation (hearing the music internally in the absence of actual sound), visualization (seeing the score or the performance situation in one's mind), kinesthetic imagery (imagining the feel of the instrument or one's body position), motor imagery (imagining the movements required, such as fingering for pianists), and emotional imagery (imagining the expressive aspects of a performance) (Fine et al., 2015, p. 70).

Auditory imagery in practice is commonly used for notational audiation and for creating an ideal image of a desired performance. Its use in performance has been shown to be justified for artistic perfection (Keller 2012, p. 206) and to be important for successful performance from memory (Highben \& Palmer, 2004, p. 58).

Keller (2012), shares that, "Robert Schumann's Humoreske, Opus No. 20 for solo piano, contains a third stave sandwiched between the conventional treble and bass staves in one section. A lyrical melodic line is notated on this extra stave, with the mysterious marking, innere Stimme (inner voice). This inner voice, which apparently represents Schumann's future wife, Clara Wieck, singing one of her own compositions, is intended to be only imagined (Ostwald, P.F., 2010, as cited in Keller, 2012, p. 208). Presumably, doing so affects the character of the parts actually played by the pianist" (Keller, 2012, p. 208). 
Hubbard's review of literature in 2010 includes a list of what is currently known about Auditory Imagery (AI). His work includes the following facts about Al (Hubbard, 2010).

1. Al can be complex, including contour, harmony.

2. Al can be generated through notational audiation.

3. Al can anticipate sound that is expected, but not yet heard.

4. Al can be manipulated in the mind, and information can be reversed or imagined in a different order.

5. Vividness has been shown to be better in auditory imagery than in visual imagery.

6. Al preserves most, but not all of the structure of the original sound including pitch, loudness, frequency of the starting tone, timbre, contour, melody, intervening beats between a stimulus, and overall tempo.

7. Because its representation is not exact, $A l$ is, instead, depictive and descriptive.

8. Al can prime a subsequent perception. It is likely to involve expectations.

9. Al can involve or evoke schemata and LTM knowledge.

10. Al Involves an overlap of brain areas with perception.

11. Al is related to musical experience, but it is not clear exactly how.

12. Al has been shown to occur in everyday life for music students, while working, interacting with other people, or during periods of waiting or prior to sleep. Most often they heard fragments of music or repeated fragments, not entire songs, mostly of music with which they were familiar or had heard recently (Hubbard, 2010, pp. 319-323).

In 2012 Herholz et al. studied memory for familiar tunes. The study showed that capacity for vivid Al and brain activity during Al were related (Herholz et al., 2012, p. 1387). They found that there was a large overlap in brain activity for imagery and perception, that is between real and imagined sound. More vivid imagers showed significantly more activity in the anterior part of the right STG, compared to those who reported weak imagery abilities (Herholz et al., 2012, p. 1391). (See Appendix B.)

With voluntary imagery, imagined sound was associated with the secondary auditory cortex and the areas around it (Herholz et al., 2012). Activity also increased in frontal and parietal areas during imagery, because frontal areas help in the retrieval of the imagined sounds and they support working memory while one imagines (2012). Motor and pre-motor areas and the prefrontal cortex (PFC), all 
areas involved with subvocalization, (see Appendix B) were active while anticipating a familiar sound one was expecting to hear (Herholz et al., 2012, p. 1382).

Research by Zatorre \& Halpern (2005) has clearly shown that there can be auditory cortex activity when there is no existing sound. (See Figure 5.) Since this is assumed to underly auditory

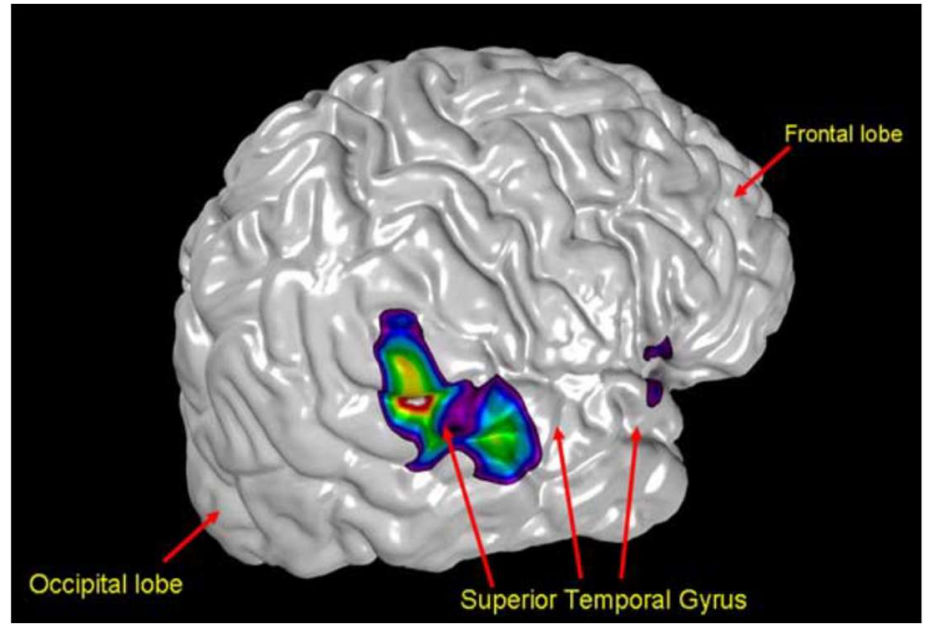

Figure 5. Lateral view of right cerebral hemisphere illustrating an area of hemodynamic increase in color, during an Auditory Imagery task. Although the task is performed in silence, activation is observed within auditory cortex in the posterior aspect of the superior temporal gyrus (Zatorre \& Halpern, 2005). imagery, Zatorre \& Halpern sought to answer the question of how the auditory cortex actually became activated (Zatorre \& Halpern, 2005). Their answer suggests that top-down mechanisms help to reactivate long-term memories, so that frontal cortical areas interact with the auditory cortex to activate it (Zatorre \& Halpern, 2005).

Interestingly, Zatorre has recently found that auditory information can be manipulated in the brain, allowing one to conceive of a phrase in retrograde or in inversion, for instance, after which a new representation becomes stored (Zatorre,

2012). The IPS (the intra-parietal sulcus) handles these manipulations (Zatorre, 2012). In Zatorre's overall findings, he shares the potential import in finding that the parietal and frontal cortices are capable of allowing past events to be generated internally and then be manipulated into something new. "In this way of thinking, then, we may have the beginnings of a model to explain some aspects of creative thinking" (Zatorre, 2012, p. 227).

Zatorre \& Halpern (2005) recounted that musicians can imagine the sound of their own instrument while using mental practice (Zatorre \& Halpern, 2005, p. 11). They further report that primary motor regions have shown activity for particular fingers that would have produced a note when a pianist listens to a piece they know how to play (Knosche, 2001, as cited in Zatorre \& Halpern, 2005, p. 11). "Imagery of related musical sounds and movement can be integrated" (Zatorre \& Halpern, 2005, p. 11).

In 2012, Halpern authored an article about dynamic aspects of auditory imagery. In her review of two particular experiments, she found that the emotional content of music can be understood and 
enjoyed, both in real time and also with just the mental imagery of a piece (Halpern, 2012, pp. 202-203). Further research by Leaver et al. (2008) showed that one can anticipate the sound of an upcoming song, for instance on a CD, by hearing the end of the song one has consistently heard prior (Leaver et al., 2008). In fact, only ten minutes of training in pairing a cue tune with a second tune provided noticeable strengthening of the brain's anticipation for the second song (Leaver et al., 2008). Halpern adds that "anticipation is likely one way that musicians calibrate their timing when playing in an ensemble" (Halpern 2012, p. 205). It makes sense that anticipatory imagery in solo piano music may also modulate timing during performance.

Keller (2012) further reports that anticipatory imagery helps in the selection of physical movements to produce (Keller, P.E. \& Koch., I., 2006a, as cited in Keller, 2012, p. 207), in knowing their order, in promoting efficiency of movement due to pre-planning (Keller, P.E. \& Koch., I., 2008, as cited in Keller, 2012, p. 207), in facilitating timing accuracy (Keller, P.E. \& Koch., I., 2006, as cited in Keller, 2012, p. 207), and in reducing reliance on tactile feedback (Keller, P.E., Dalla Bella, S., \& Koch., I., 2010, as cited in Keller, 2012, p. 207).

Because imagery is a complex construct of the mind, a number of scales have been constructed as scientific means to understand its existence and to empirically quantify and define it. The newest of these scales, the Bucknell Auditory Imagery Scale (BAIS) for sound, developed in 2012, has been shown to be scientifically reliable and can be used for visual imagery, auditory imagery, and environmental sounds (Halpern, 2012, p. 37). The scale consists of 28 self-report items that participants rate based on the vividness of an imagined sound and the ability to imagine the sound changing (Halpern, 2012). The BAIS system is used regularly in research and in much of what follows here, because it addresses imagery in quantitative and qualitative ways that have scientific reliability.

\section{Imagery in Performance. A Dualism?}

In 2015, Saintilan suggested a dualism in piano performance that "has been described as an intermediate step between thought and production of external sound, giving rise to the possible use of multiple imagery types that musicians can use to construct this intermediate step" (Saintilan, 2015, p. 311). In other words, a performer's auditory image acts like a performance plan, and its inner performance experience happens first, immediately prior to a second, public one. 
For example, in a study of self-reporting by members of the Chicago Symphony brass section, one musician stated, "If I don't hear it [the ideal sound] or conceptualize it in my brain, there's no way I'm going to get it" (Trusheim, W.H., 1993, as cited in Keller, 2012, p. 208).

In 1986, The Inner Game of Music author and bassist, Barry Green, writes, "effectively, you are playing a duet between the music in your head and the music you are performing. Any notes you play that don't correspond to your imagined sense of the music stand out, and your nervous system is able to make instant, unconscious adjustments" (Green, 1986, p. 75). His statement implies that there is a small time gap available for mental comparison between the imagined sound and the yet-to-be live sound. Psychologist William James' understanding was slightly different, when over a century ago he stated, "we [musicians] are then aware of nothing between the conception and the execution. All sorts of neuromuscular processes come between. . . but we know absolutely nothing of them. We think the act, and it is done" (James, 1890, as cited in Keller, 2012, p. 790). James implies that aiming for the goal and its occurrence happen so succinctly as to seem simultaneous to the performer.

An experiment by Saintilan in 2015 intended to find whether musicians are always using inner singing, or some form of intermediary step that produces the duality of an inner performance prior to an outward one. String players, a horn player, a bassoonist, and two pianists were interviewed, all of whom described some form of inner image in performance. Some described it as hearing, while others described it as singing. For example, string players reported, "I did sing the piece internally," or "[I] basically use subvocal singing the whole time" (Saintilan, 2015, p. 312). Pianists reported, "what I imagine is tone color, the sound..." or "what I imagine is tone color, the sound, and technique required to produce the desired color" (Saintilan, 2015, p. 313). For the types of imagery participants reported, see Figure 6. 


\begin{tabular}{|c|c|c|c|c|c|c|c|c|}
\hline Musician & Auditory & Kinaesthetic & Spatial & Timbral & $\begin{array}{c}\text { Visual } \\
\text { Notation }\end{array}$ & $\begin{array}{c}\text { Visual } \\
\text { Analytical }\end{array}$ & Verbal & Emotional \\
\hline VIn 1 & $x$ & -- & -- & -- & -- & -- & -- & $x$ \\
\hline VIn 2 & $x$ & $x$ & -- & -- & -- & -- & - & - \\
\hline VIn 3 & $x$ & $x$ & -- & - & 0 & 0 & $x$ & - \\
\hline Vcl 1 & $x$ & -- & -- & -- & $x$ & -- & -- & -- \\
\hline Vcl 2 & $x$ & $x$ & -- & $x$ & -- & -- & $x$ & -- \\
\hline Pno 1 & $x$ & $x$ & -- & $x$ & $x$ & A & -- & -- \\
\hline Pno 2 & 0 & A & A & -- & $x$ & A & -- & -- \\
\hline Bassoon & $x$ & $x$ & A & - & 0 & 0 & -- & -- \\
\hline F. Horn & $x$ & -- & -- & -- & 0 & -- & $x$ & -- \\
\hline
\end{tabular}

Figure 6. Key to symbols: Experienced $=\mathrm{x}$; Inexperienced $=0$; Ambiguous response $=\mathrm{A} ;--=$ No imagery type mentioned (Saintilan, 2015).

Musicians are clearly using multimodal imagery. But are there really two performances always occurring? If one sings along with a performance, is the inner singing really happening first, or is the performer not consciously aware that two events are happening?

Saintilan proposes that pianists may not fully hear the first internal version due to prioritizing the outward, public one (Saintilan, 2015, p. 310). However, if one is aware that two separate events are happening, could there be a method by which the duality becomes a unified act of doing?

One major problem with the duality theory is that sound imagery is unlikely to be the only kind of imagery a performer uses. Current research supports the idea that sound imagery is probably impossible to separate from physical imagery of the necessary motions involved in performance. Pianists are likely to experience sound imagery in connection with the motion imagery involved (Saintilan, 2015, p. 310).

\section{Multimodal Imagery in Performance Preparation}

The duality question may be related to how one prepares to use multimodal imagery in performance. Davidson-Kelly et al. (2015) undertook a five-day masterclass as an experiment centered around multimodal imagery in concert with the precepts of Alexander Technique. 
In these classes, taught by an experienced Alexander Technique instructor, the goals were to find how 11 pianists experienced and employed imagery techniques, as well as how they would describe the Alexander pedagogy (Davidson-Kelly, 2015). Leaders hoped to add to real-world knowledge of how imagery and multimodal preparation was beneficial to music memorization and performance.

Sessions focused on creating a clear image of the piece to be performed via multimodal imagery techniques and chunking strategies (Davidson-Kelly, 2015). Alexander Technique theorizes that if imagery rehearsal is clear and purposeful enough, it may strengthen the connection between one's intention and one's actual performance. The concept of total inner memorization required that a piece be clearly memorized internally before any physical practice could take place (2015).

The course taught "a sequence of deliberate imagery strategies for learning new material, for dealing with technical difficulties, and for enhancing performance" (Davidson-Kelly, 2015, p. 85). Alexander Technique teaches that one should make the simplest and clearest physical motion, devoid of unnecessary movement or tension, that achieves the musical goal. Overall, they envisioned the process of mental practice as an active and creative one, keeping in mind that mental imagery transfers to performance and, further, that mental and physical activity cannot be entirely separated (2015).

Participants found that total inner memorization required enormous amounts of will, concentration and patience; those with little experience in using imagery could not do it at all (Davidson-Kelly, 2015). The difficulty level of this technique was a setback to the outcome of the experiment (2015).

In the end, pianists reported different balances of how much physical practice they used, how they avoided technical problems, how much aural or motor imagery they used, etc., during the five days of sessions (Davidson-Kelly, 2015). Many reported that Alexander Technique's physical approach helped them to avoid technical issues, to think more clearly, and to engage more closely with the music (2015). In the end, however, none of the participants incorporated the teacher's plan in full or in its presented order (2015).

The authors described their goal as wanting to add to real-world application knowledge of imagery in memorization and performance. They concluded that "deliberate mental rehearsal of multimodal, structured units within a large, complex musical piece might lighten the cognitive load in recalling the music and assist in producing efficient and fluent movements in performance" (DavidsonKelly, 2015, p. 90). Further study is needed, however, because these results were rather inconclusive, and the number of participants was somewhat low (2015). 
This study did seem to show that balancing mental imagery with physical practice was desirable, and that even a small amount of rebalancing, or the injection of imagery practice, for individual participants was helpful to them. Although developing imagery skills sometimes requires effort, these skills may ultimately lead to improved security, improved memory, and therefore, optimal music performance (Davidson-Kelly, 2015).

\section{Sequential Planning, Prosody, Hierarchy, and Units of Learning}

While imagery plays a key role in piano performance, music and physical motions are still accomplished in sequential time. A performer imagines the music and the motions that will be required during performance. The imagined is brought to realization, while at the same time the performer is planning for the next musical event. It is now known that correct auditory feedback is essential for planning in piano performance. Research has shown that musical structure and the way one understands it affect perception and learning, and that recall relies on these same mental structures. Further, a performer's individual view of musical structure provides the basis for musical expression. More recent research has uncovered interesting aspects of how planning happens during performance, including how far in advance it may be done, and how planning and timing may interact with error.

\section{Sequential Planning - Planning for what comes next.}

In 2016, Mathias et al. investigated how planning and feedback monitoring interact with each other. During performance, one is continually planning for the future while one is playing (Mathias et al., 2016). Planning for the next event in performance happens simultaneously with hearing current feedback, the sound of what has just been played. This process was studied by manipulating feedback that pianists heard while they performed.

Skilled musicians played short melodies, and were occasionally given incorrect, altered feedback pitches: pitches that had already happened in the recent past, pitches that were upcoming in the near future, or non-contextual pitches that were taken from the key of the passage (Mathias et al., 2016, p. 243). The altered feedback pitches represented naturally occurring mistakes in piano performance. It has been shown that mistakes tend to be comprised of just one note, and almost all are from pitches used in the nearby musical area (Mathias et al., 2016, p. 244).

Results indicate that only altered feedback from future pitches was disruptive to the pianists' performances (Mathias et al., 2016). There was no disruption at all for altered feedback from the past, 
or from non-contextual pitches. In all cases, the brain did recognize that altered feedback occurred by eliciting electrical responses that may not have disrupted performance (Mathias et al., 2016).

The amount of disruption that occurred and the amount of time needed to play the note after the altered feedback were both related to the neural potentials observed (Mathias et al., 2016). The results show that planning is related to the future events, not to past ones, and that correct feedback is related to planning (Mathias et al., 2016).

\section{Sequential Planning: Near-future and far-future events.}

In 2019, Mathias et al. studied the sequential planning aspects of performance by altering feedback. Feedback was altered by using near-future pitches about to be played or using far-future pitches that would not occur subsequently. The near-future altered feedback was disruptive to performance, but the far-future feedback was not (2019). This strongly suggested that greater interference in sequential planning occurs with near-future interference.

"Musicians possess strong associations between musical actions and their sensory outcomes, which may explain why the perception of inaccurate auditory feedback during the production of auditory-motor sequences can disrupt production" (Mathias et al., 2019, p. 2).

Past studies of error production for both music and speech have shown that there is a range of future events to which one has access for planning during sequential performance (Mathias et al., 2019, p. 1). Other recent research, together with this particular study, suggests that pianists have more planning access to events that will happen in the near future than to those in the far future (Matthias et al., 2019).

Further, this research hypothesizes that a disruption for future events is on a gradient that decreases the further away the incorrect feedback is from the present (Mathias et al., 2019). If so, future research may be able to determine specific conditions for the timing of event planning during performance. This could provide evidence that focusing on the very next event, and only the very next event, is optimal, and that the very next event may be as large as an entire phrase, or the amount of a sequence that can be currently stored in working memory.

\section{Auditory feedback is essential for sequential planning in performance.}

In 2010, Furuya \& Soechting investigated the effect of altered feedback on the rhythmic timing and velocity of upcoming sequential keystrokes that a pianist makes. They altered auditory feedback either by changing the pitch, the loudness, or the timing of one note, while pianists played excerpts at slow, medium or fast speeds. If the pianist heard a delay, the next keystroke was made sooner than usual (2010). If the pianist heard a delay or a wrong pitch, the speed of the next keystroke increased, but 
an alteration in loudness changed the next keystroke's velocity in various ways (2010). Altering a pitch from the right hand caused changes in the left-hand reaction as well, so that the coordination between hands was preserved. If the pianist heard a wrong pitch, often a wrong note followed. These findings point to auditory feedback as necessary for controlling timing and accuracy in sequential piano performance (Furuya \& Soechting, 2010, p. 223).

\section{Hierarchy and Units of Knowledge.}

In 1993, Palmer \& van de Sande investigated the kinds of information storage that musicians use in performance by assessing the quantity and types of errors that tended to occur in performance. Errors help to demonstrate what a performer had prepared or was planning, and therefore aid researchers in finding neural connections. Because units of information are stored in memory and later retrieved, researchers investigated the size and kind of units these might be (Palmer \& van de Sande, 1993).

It was assumed that different textures and musical situations would form different structures and different prioritizations for units. For that reason, homophonic music, polyphonic music, sightreading material, and learned music were all studied (Palmer \& van de Sande, 1993). To assess the advantage of the uppermost right-hand voice, melodies were placed in different places and in different hands, and pianists were instructed to play using different interpretations. Several different kinds of error (for example, mistakes of omission or mistakes by replacing a note with a wrong note, etc.) were identified in advance so they could be classified (1993).

The results showed that errors in homophonic music were mostly related to chordal units, and errors in polyphonic music were mostly related to single-note units (Palmer \& van de Sande, 1993). Overall, errors were related harmonically or diatonically to the correct notes, thus demonstrating that memory retrieval was based on planning. The line in the uppermost finger of the right hand, melody or not, showed the fewest errors, demonstrating a prioritization for what is heard on the top (1993).

"These findings suggest that retrieval processes during music performance may reflect the same relationships among levels of musical structure as do encoding processes during perception. For example, the different error sizes and sources of influence support the assumption that performance plans are constructed from multidimensional relationships among melodic, harmonic, and diatonic elements" (Palmer \& van de Sande, 1993, p. 467). Because the key of a piece is strongly related to standard chords within it, and chords, in turn, are strongly related to their component pitches, errors more commonly confuse scale notes in a piece, or chordal pitches inside chordal areas (1993). It is also 
posited that melody is well attended in practice, thereby reducing errors for melody in performance (1993).

It should be noted that homophony, polyphony, and melody are not the only definitions of musical units of knowledge. In 1995, a subsequent study by the same research team noted that performers partition sequences for performance by breaking them into shorter pieces and, further, that phrase structure often influences the choice of partitioning (Palmer and van de Sande, 1995). They found that errors within a phrase were more likely to be pitches from that phrase than pitches existing outside it (Palmer \& van de Sande, 1995, p. 121).

\section{Planning, Prosody, Hierarchy, and Timing.}

In 1997, Palmer sought more information about sequential planning, timing, and memory in performance. While we may think of learning lengthy strings of information partly by associative cuing, Palmer concludes that considering "chain-like organization of behavior is inadequate to explain certain serial order effects in sequence perception and production" (Palmer, 1997, p. 121). If a pianist simply played learned sequences in order, they would not make sense because the rhythmic component would be missing. Therefore, both serial order and relative timing of sequences are constraints to be met (Palmer, 1997, p. 117).

"Planning and memory retrieval processes in music performance reflect multidimensional relationships among melodic, harmonic, and diatonic elements. In Western tonal music, individual pitches, chords, and keys are posited as conceptually distinct units of knowledge, that reflect levels of melodic, harmonic, and diatonic structure, respectively" (Palmer, 1997, p. 121). Accent structures in music are another way that segmenting happens, in that it has been found difficult to reproduce a melody if it is shifted from its regular metrical structure (Palmer, 1997, p. 122).

Memory capacity probably places constraints on the distance between what one is currently playing and what one can plan. But also, structural boundaries, like the ends of phrases, tend to determine how far ahead one can plan (Palmer, 1997). In early sight-reading studies from the 1970s, eye-hand span tests were performed where a passage was briefly played and shown to a pianist, but the score ended within the passage. It was found that participants could remember about seven or eight events past where the score stopped, but memory tended to end at the end of a phrase if this occurred first (Palmer, 1997, p. 122). Palmer finds that in memorized music, planning could happen for events further away if there were no intervening phrase boundaries (Palmer, 1997, p. 122). 
Palmer suggests two potential rules about planning complex sequential behavior. First, events with the same structural features may be planned at the same time. Second, structural boundaries constrain the serial distance over which planning can happen (Palmer, 1997, p. 122).

The syntax and stylistic traits related to structure have an impact on sequential planning in performance. Generally speaking, Western music is inherently hierarchical, both metrically and tonally, which predicts that "more important events are processed at deeper levels and thus memory should be facilitated for those events" (Palmer, 1997, p. 122). This requires the organization of practice around musical structure, with clarity as to which structural events are hierarchically deeper than others.

Rhythmically, musical events that happen with the tactus are commonly emphasized in performance. As to sequential structure, the phrase is thought of as being the most salient, perhaps because it is the largest grouping that fits in short-term memory (Palmer, 1997, p. 122). It is clear that learning, memorization, performance planning, and expression all concern phrasing (1997).

Understanding the structure of a piece of music is crucial not only for its memorization, but also for its recall. From this study, pianists may understand that the deepest points of underlying music structure must be thoroughly understood in order to perform optimally (Palmer, 1997).

\section{Hierarchy and Individuality.}

In 2016, Gingras et al. studied melodic emphasis, texture, and performer individuality in order to understand more about performance errors. A Bach fugue and two short Baroque organ pieces were used that included both homophonic and polyphonic styles. Results were generally in line with Palmer and van de Sande (1993). In the fugue, areas comprising musical motives and outer voices demonstrated fewer errors by the participants (2016). Melodic emphasis had a direct effect on the location of errors, related to which voice was carrying the melody at the time of the mistake. The musical texture affected what kind of error occurred (2016).

Overall, each performer's errors were more consistent with their own error patterns than with errors across the other 23 participants. Indeed, "this suggests that individual structural perceptions were involved with planning and interacted with produced errors. These findings suggest that error patterns, like timing, articulation, or intensity change patterns, are shaped by a performer's unique conception of a score and of its musical realization" (Gingras et al., 2016, p. 861).

\section{Planning while Performing.}

In 2012, Palmer et al. studied pianist's actions that go partially wrong. For this study, a partial error was defined as a correct pitch with an incorrect force or duration. By examining what happened 
prior to a partial error, they were able to learn about what had been planned for the next sequential movement.

A partial error usually occurred with less force, with slower key descent and softer dynamic level, than correct motions (Palmer, 2012). The event prior to it "tended to have durations and intensities that fell between those of errors and correct responses, presumably due to response competition with upcoming errors that resulted in partial-error outcomes" (Palmer, 2012, p. 185). Preerror events take on some unwanted properties of the expected error that has not happened yet. For pianists, this study confirms that one is always planning the next motion while performing the current one and that the two events do interact (Palmer, 2012).

\section{Informational Units in Memory and Performance.}

In 2017, Moldwin et al. studied melodic patterns in order to improve understanding of how the brain stores auditory memory traces. Participants heard a melody and then were presented with the same melody with imposed wrong notes. They found that the brain's responses to wrong notes increased if the wrong note was within a phrase, rather than between phrases, where the structure of the melody would be understood (Moldwin et al., 2017). This indicates that auditory memory traces keep a melodic pattern or phrase in short-term memory at one time and that these patterns or phrases become the permanently stored units. The stored units are "useful for perception" (Moldwin et al., p. 2120). While it is commonly believed that this is how STM operates, this very recent study "demonstrates that informational units form the basis for processing the auditory scene and detecting novelty within it" (Moldwin et al., p. 2121). If musical structure and its storage are the basis of perception, they must also be the basis for retrieval. Current performance research continues to investigate how these informational units are retrieved in sequential order and with correct timing.

\section{Hierarchy and Musical Expression.}

Palmer (1997) contends that expressive timing choices are also based on structure, due to the amount of research showing a causal relationship (Palmer, 1997, p. 123). Music structure is the reason for expressive choices. Rubato is often used to show a hierarchy of phrases: phrases often slow at the end, and more important phrases slow down more than others (Palmer, 1997, p. 124). Metrical accents are often louder and given a lengthier duration. This is easily demonstrated when a group of people repeat a pattern of four for a reasonable length of time: the strongest accent occurs naturally on beat one, with a secondary accent on beat three. Rhythmic flexibility and expression due to structure can involve both accent and lengthened beats (Palmer, 1997). 
Palmer (1997) also notes that expression can be used to present interest and intertwining of different voices in polyphonic music. In polyphonic music, this is especially important because the listener can only attend to one voice at a time, so the performer must choose what will be highlighted for the listener's attention (Palmer, 1997).

Palmer underscores that "many findings have established a causal relationship between musical structure and patterns of performance expression" (Clarke, 1988; Palmer, 1989; Sloboda, 1983; as cited in Palmer, 1997, p. 123). But past studies show that structure cannot be the only source of expressive interpretation (Clarke, 1993, Clarke \& Baker-Short, 1987, as cited in Palmer, 1997). In these studies, pianists performed timings that conventionally related to hierarchical phrase structure with high accuracy, but they were still able to perform expressive gestures that had distorted or arbitrary relationships to the structure. Accuracy in the latter became more difficult as the structure-expression distortion increased, yet the tested pianists continued to improve with practice. "Evidence that performers can imitate expressive timing patterns that have an arbitrary relationship to the musical structure suggests that performance expression is not generated solely from structural relationships" (Clarke, 1993, as cited in Palmer, 1997). Further research may demonstrate other components of expressive timing choices, which may likely include individual differences.

\section{$\underline{\text { Imagery in Performance and Preparation. Mental Practice }}$}

Most musicians regularly use some form of mental practice. In the common practice of notational audiation, it has been found that producing correct auditory imagery is correlated with overall ear-training skills and is correlated very little with working memory ability (Highben \& Palmer, 2004). Even analytical study of a score, when not intentionally creating a mental sound, can lead to auditory or motor imagery (Highben \& Palmer, 2004).

In 2015, Fine et al. studied forms of mental practice in 89 musicians alongside types of score analysis reported earlier (See p. 27.) In their own words, musicians in this study reported mental practice as "occurring away from the instrument; involving several types of imagery, happening often in real time; and focusing on performance preparation, particularly aspects of execution and realization. During mental practice, the score tended to function more as a memory aid, an orientation guide, and as a point of reference for interpretation. Score analysis was considered to be more theoretical, though still relevant" (Fine et al. 2015, p. 69). 
While score analysis was deemed more useful for the early understanding of structure, mental practice was reported as being useful from the first reading of a piece to its final performance (Fine et al., 2015). "According to interviews with conservatoire-level musicians, mental practice is considered useful for-developing emotional expressivity; enhancing technique and practice efficiency; improving learning and memorization; heightening sensory awareness; refocusing attention during performance; enhancing general confidence and resilience in a performance situation; enabling greater control over negative emotions; establishing a stronger connection with an audience; and achieving peak experience" (Fine et al. 2015, p. 71).

The concepts of inner speech, the inner ear, and subvocalizing are related to mental practice, implying that the system one uses to create speech by moving one's mouth is being used while one imagines (Saintilan, 2015). The inner voice, or inner singing, can occur in the mind with no movement of the mouth or imaginary movement of fingers or arms. In a related finding, Hubbard reported that preventing subvocalization has been shown to inhibit auditory imagery (Hubbard, 2010, p. 314).

In the research by Fine et al. (2015), audiation and the imagining of actual motor skills in performance were the forms of mental imagery mentioned most, along with visualizing the actual score and imagining multimodal aspects of playing. The goals for mental practice were ultimately about performance optimization. Imagery is clearly of vital importance to achieving this (Fine et al., 2015).

In 2004, Highben \& Palmer (2004) desired to study only auditory and motor mental practice as the two forms most typical in non-physical piano practice. In physical practice, pianists receive both aural feedback by hearing what they are playing and kinesthetic feedback from what they feel while performing motor movements. This research showed that removing either of these two kinds of feedback during practice had a detrimental effect on learning, even when both kinds of feedback were available in the performance situation (Highben \& Palmer, 2004).

In this experiment, pianists possessing strong ear-training skills had fewer performance errors than others when no feedback had been available during practice (Highben \& Palmer, 2004). This was also true for pianists who self-reported that they often played by ear (Highben \& Palmer, 2004). Further, they found that "individual differences suggest that performers differ in their motor imagery and learning versus their auditory imagery" (Highben \& Palmer, 2004, p. 64). Auditory imagery and motor imagery are both very common and they were found to interact with each other (Highben \& Palmer, 2004).

Overall, and most fundamental to pianists, this research correlates a pianist's clear auditory image to their ability to learn a new piece from a score without being able to hear their own practice 
(Highben \& Palmer, 2004). The research concludes that having a correct auditory image is important to the ability to perform from memory successfully (Highben \& Palmer, 2004).

In 2005, Holmes studied the preparation habits of a professional cellist and a professional guitarist, noting that excellent practice habits for preparation to perform by memory are applicable to all instrumentalists, and that they are always generated by interpretive goals. She found that playing goals were met by using intuition that involved imagery, and that motor imagery was highly significant. Although results in this study are similar to others already described (e.g., Lisboa et al., 2018; Chaffin et al., 2016), Holmes found that imagery associated with both technical and emotional aspects of the music, together, helped the music to become more deeply entrenched in memory (2005). This surely relates to the memory-strength component of Soderstrom \& Bjork (2015), who claim that memories' extended relationships to other memories improve their long-term strength. More closely related are Tania Lisboa's performance cues, linking musical emotion to motor action (Lisboa et al., 2018, discussed on p. 43). In this study, Holmes connects the use of emotional and motor imagery, together, as a strengthening agent for memory in performance (Holmes, 2005).

In 1997, Palmer wrote that the more science uncovers about perception and performance, the more the two seem to be tied together, such that the "distinctions between the psychological mechanisms proposed for music perception and performance are becoming blurred" (Palmer, 1997, p. 134). Here, Palmer considers the idea of melody as key to how auditory scene analysis behaves, in both music perception and music performance, because melody is a foremost element in capturing our attention and our imagination. "Melody - the primary voice that grabs listeners' attention, sticks in performers' minds, and arguably is conceived first in multi-voiced music - may be the reason why music exists" (Palmer, 2015, p. 9). How does a performer use melodic imagery in performance?

Palmer concluded that "our percepts for melody are privileged" (Palmer, 2015, p. 6). In performance, one's attention and focus are often placed primarily on the melody, and rightly so. An important consequence of this prioritization is that one can be altered by it (Palmer, 2015).

To support this idea, Palmer found that "individuals alter their behavioral and neural representations of music during perception, depending on prior performance experience with that melody" (Palmer, 2015, p. 7). In other words, those who have performed a melody have a special and different relationship with it than those who have only listened to it. A specialized auditory-motor connection is created when both hearing and motion are involved (Palmer, 2015). While it has not been proven, this connection may be very different from one that forms either from listening or from motion alone (Palmer, 2015). 
Not only did pianists more easily recognize melodies they had performed over ones they aurally knew, but also, EEG experiments have shown that behavioral and neural changes reflect stability of melodies after musicians have performed them (Palmer, 2015, p. 8). Performing or creating systematically alters the musical experience of melody. Performance experience creates lasting mental changes (Palmer, 2015, p. 8).

Baumann et al., (2007) sought to explain more about how imagery affects brain activity in performance for musicians and non-musicians. In pianists, they found clear evidence that secondary auditory and motor areas exchange information during piano performance, including the dorsal premotor cortex, the planum temporale, and the planum polare (Baumann et al., 2007, p. 69). Piano performance activates an audio-motor network of secondary and higher-order structures which are tightly interconnected (Baumann et al., 2007). (See Appendix B.)

\section{Working Memory and Focus of Attention in Performance}

Interpretation in performance lends itself to expressive timing that causes rubato and irregularities in the regular beat. It has recently been found that working memory and auditory imagery each have roles to play in feeling and anticipating expressive timing (Colley et al., 2018). Research often studies sensorimotor synchronization (SMS), the way that a person can synchronize with audible music, to study expressive timing. Successful SMS depends on timing motor output with music, or timing that is happening outside oneself (Colley et al., 2018). This would concern playing in ensembles, accompanying, performing a concerto, etc.; expressive timing in solo playing is likely to behave similarly.

In real time, this process requires planning and complex motor-control systems. However, imagery allows one to imagine motions that are necessary-and their potential consequences-before an action plan is made. "Indeed, there is evidence that 'replaying' internal auditory experiences is part of the process of planning and executing actions. For example, being able to imagine a sound and simultaneously map it to a specific action sequence produces more efficient action execution" (Colley et al., 2018, p. 1783).

Working memory continually updates timing intervals in music performance in real time, as one plays (Colley et al., 2018). Along with timing updates, working memory receives long-term memory of music structure or patterns, including imagery about what has been practiced, and it uses all this information to create an action plan. Therefore, working memory provides the ability to feel expressive timing patterns; further, working memory may also be the basis for being able to maintain imagery in 
active use (Colley et al., 2018). The results of this study suggest that having vivid, clear imagery may aid in judging aspects of real-time, incoming sound. Because working memory mediates how one feels specific timing cues for real-time expression, attention and focus are important to a successful performance.

A related study by Duke et al (2011) directed pianists' focus of attention toward different aspects of their playing in order to observe the effects. College music majors, including pianists, were asked to attend to either their fingers, the piano keys, the piano hammers, or the sound produced when playing a brief piano passage. The results were analyzed using MIDI data and software specific to this experiment (Duke et al., 2011, p. 44).

No significant results were found in the retest, but significant results were found in the transfer test, where the participants were asked to perform motor skills that were somewhat different from the training skills (Duke et al., 2011, p. 51). Their findings suggest that placing the focus of attention on resultant sound does not immediately affect one's performance, but it does increase learning. This study was important in demonstrating that shifting a performer's focus toward the goal and away from the motions required to achieve it enhances learning. Moreover, the study demonstrated that "the more distal the focus of attention, the more accurate the motor control" (Duke et al., 2011, p. 44).

This test supports the concept that focusing attention on the product of a pianist's movement, the desired sound, is more effective than focusing on the movement itself. Relying on some portion of automatic and well-practiced motor processes by focusing attention away from the motions themselves allowed for more successful performance (Duke et al., 2011).

A 2015 study by Maes et al. showed that working memory capacity was reduced when musicians used discrete rather than continuous movements. Working memory was also shown to produce errors and timing problems when melodies by cellists were performed with discrete bowings (Palmer, 2015). Therefore, this study suggests that continuous body movements provide better timing control for musicians in high-stress situations like performance, where working memory load is high (Palmer, 2015). Though it has not been studied, it seems reasonable to infer that working memory load for pianists may be stressed in long passages of playing staccato. This may become the science behind why practicing staccato passages in legato is important in giving oneself the impression of continuous movement. Conversely, practicing legato passages in staccato may increase the difficulty level significantly, making the intended legato seem much easier. 


\section{Music Embodiment}

A 2016 editorial by Cohen reviews interest in the relationship of music to non-auditory domains beginning in the 1990's, and that more recently, the concept of music embodiment has taken root. Because it is increasingly understood that music is "inherently multimodal" (Cohen, 2016, p. 99), its holistic aspects have propelled music embodiment to become a more prominent field of study.

\section{The Concept of Music Embodiment.}

Some primary research in music embodiment is currently taking place in Ghent, Belgium, by Marc Leman, P. J. Maes, and their colleagues. The music-embodiment approach places emphasis on the role of the human body and its actions as mediation for understanding musical meaning. As such, it has been called "the pragmatic turn in cognitive science" (Engel, Maye, Kurthen, \& König, 2013, p. 202 in Leman \& Maes, 2014, p. 236). Music embodiment implies that the human motor system, its gestures, and body movements play an important role in music perception (Leman, 2007; Godøy \& Leman, 2009). While it has always been thought that a person encounters music and develops a mental perception of it, which may in turn effect bodily movement, it is now believed that the process also works the other way around: bodily movement and response to music are key to its complete understanding and perception. Therefore, at its heart, music embodiment assumes that music or a musical environment provides physical information to the body, which reacts and informs a person as to its meaning (Leman \& Maes, 2014).

New research technologies in recording human movement have allowed music embodiment to become a leading field of study in the last decade. (Leman, 2007). Current research in what is often referred to as action-perception couplings, that is, the co-dependence of bodily action with music perception, is now able to provide practical empirical results. Importantly, research suggests that the "affect of [physical] action on music perception is essential in making sense of music" (Maes, Leman, Palmer, \& Wanderley, 2014; Leman \& Maes, 2014, p. 237). In other words, gestures that are driven by hearing or interpreting music are now thought of as being integral to musical perception (Leman \& Maes, 2014).

\section{Musical Expression and Gestures in Music Embodiment Research.}

As music embodiment is deeply related in a physical way to traditional music performance, musical expression has become a key area of its research. Researchers assume that people have "mirroring processes" (Leman \& Maes, 2014) that allow them to create physical responses that directly interpret sound, and vice versa, to encode expressive gestures into sounds. This research intends to 
understand gesturing as a way of interpreting music while performing and also as a way of interpreting music as an observer of gestures, or as a listener. Therefore, gestures are a common and important factor for study, including everything from basic gestures to ones that specifically support sound, specifically support expression, and specifically respond to expression. Commonalities among gestures of musicians and non-musicians may become an interesting aspect of modern research findings.

\section{Predictive abilities and attention.}

One's particular physical and mental states of being have an effect on the activeness a person has in making predictions during musical interaction. This affects how one will perceive music. For example, research has found that selective attention can focus perception toward specific aspects of musical content, perhaps modulating one's broader perception of a piece (Leman \& Maes, 2014). Therefore, music cognition must involve one's state of being as it encounters a "person-with-music" interaction. In the view of music embodiment, this involves not only one's mental function, but one's physical effort and emotion, as well (Leman \& Maes, 2014). Therefore, a person's state of being at the time of experiencing music is considered to be an important factor in modern research.

\section{Music embodiment as essential.}

In further support for the study of music embodiment vs. traditional study of music perception, research in dance has been performed where people were induced into states of varying emotion, such as happiness and sadness, prior to observing their movements to music. Results demonstrated that "based on the induced emotional state, people's perception of the music was different, and it was

obviously reflected in their bodily dance movements (Godøy \& Leman, 2009; Maes, et al., 2014; Van Dyck et al., 2014). The authors conclude that "a perception only approach [to research] is no longer defensible (Leman et al., 2018, p. 751). Music interaction is based on action-perception coupling, integrated within the [brain's] predictive machinery, and above all, tightly integrated with the corporeal mediator constraints" (Leman et al, 2018, p. 751). Leman et al. (2018) remark that "the focus on cognition in perception has been replaced by a focus on cognition in interaction, of which perception research is a part" (Leman et al, 2018, p. 757). This line of research is key to watch.

As a primary example, in 2017, Hansen \& Oxoby wrote about two pilot studies in the field of embodied learning and its potential relationship to the performing arts. Using performance generating systems, a specific format that creates improvisational situations, they desired to "understand the complexity of the arts and its cognitive and learning effects" (Hansen \& Oxoby, 2017, p. 77).

This research was led by Pil Hansen, known as a cognitive performance studies scholar and dramaturge. Her "research projects examine layers of memory and perception in creative processes 
through interdisciplinary behavioral experiments" (University of Calgary, 2021). For these experiments, she collaborated with a number of other research assistants and professionals in the arts. Their hypothesis stated that, "active participation in performance generating systems has a positive effect on advanced student performers' working memory capacity, executive functions, and learning" (Hansen \& Oxoby, 2017, p. 77).

Hansen explains that "within a Performance Generating System . . performers must work attentively and simultaneously on multiple tasks while remaining aware of limiting rules" (Hansen \& Oxoby, 2017, p. 77). It is designed so that patterns of interactions take place that cannot be predicted or exactly repeated, and further, the interactions required sustained attention to different foci (2017).

In this instance, most of the work was done by evaluating interactions of people collaborating onstage under a strict system of rules and constraints. Yet, people from all areas of performing arts participated, and the lessons learned may be transformative and applicable to all areas of performance.

The primary example of their Performance Generating System concerned "futuring," where participants were asked to dance in unison with someone whose choreography they did not know (Hansen \& Oxoby, 2017). At the same time, they were required not to copy the leader, nor each other, but to concentrate on anticipating where the leader and the group would be in the ensuing moment. This partial explanation is a simplification of a system which amounts to a set of rules that were basically impossible to sustain. People reported that the process took tremendous energy and focus. They had to regroup, for instance when they caught themselves copying or could not remain focused on the whole experiment. Further, when the group began to adapt to the rules, new ones were added so participants could never become acclimated to the system (Hansen \& Oxoby, 2017).

The results of this line of research could eventually be pivotal, because the goal does not require "getting out of one's head"; instead, it intends to "train a mindful body and embodied mind" (Hansen \& Oxoby, 2017, p. 80). One researcher claims that when using these systems, "thinking is doing, and doing is thinking" (Hansen, Kaeja, \& Henderson, 2014, as cited in Hansen \& Oxoby, 2017, p. 80).

These practices may "[push] past the Cartesian body-mind dualism found in some dance, theatre, and performance studies frameworks" (Hansen \& Oxoby, 2017, p. 82). "Body-mind dualism is so engrained in social practices within and beyond dance and performance that direct attempts to apply theories of embodied cognition can come up against boundaries" (Hansen \& Oxoby, 2017, p. 80). This research reports that their research field is growing, but that little research has happened on methods of challenging mind-body dualisms in creative practices (Hansen \& Oxoby, 2017, p. 80). 
While results of this complex study were difficult to quantify, the participants' relationship to different kinds of learning was found to change over time (Hansen \& Oxoby, 2017, p. 89). It was also concluded that "performance generating systems helped participants push past a hierarchical relationship between conceptual and experiential learning and instead establish a reciprocal relationship between the embodied understanding of concepts and conceptual engagement with movement" (Hansen \& Oxoby, 2017, p. 90).

\section{Motor Skills in Performance}

A 2016 study by Bianco et al. tested whether musical syntax plays a role in motor movement behavior. Using ERP's, (see Appendix A) they were able to isolate action planning in pianists that was related to syntax vs. action planning that was not related to syntax (Bianco et al., 2016). For this experiment, syntax was defined simply by the identity of the final chord of a typical progression. Would the final chord be expected by musicians or not?

Results demonstrated that syntactically unexpected chords produced a delay in performance (Bianco et al., 2016). Alongside this delay, an ERP negativity in the central parietal lobes demonstrated that a syntactically congruent, expected movement had already been planned by the brain in advance. Because experienced pianists have learned common musical syntax (in this case, cadences), their brains can produce motor predictions about normal situations, including the automatic selection of standard fingerings (2016).

In this experiment, the higher, hierarchical goal was settled first, after which a lower movement was primed to happen (Bianco et al., 2016). In other words, motions for unexpected final chords demonstrated that a motor plan for the anticipated chord had been pre-planned. In this experiment, it is worth noting that different electrical signals in the brain occurred for unexpected chords vs. simple mistakes, which further suggests that these two factors also operate on different hierarchical planning levels (Bianco et al., 2016).

Full results demonstrated that motor plans operate at the level of musical syntax and are incrementally translated to lower levels of movement selection (Bianco et al., 2016, p. 41). This study confirms that higher-level hierarchy in movement is goal-related, while lower-level hierarchical movement concerns the action needed to meet the goal (2016). In summary, the goal of an overall motor action took priority over the particular, smaller, or later-selected movements involved (Bianco et al., 2016). 
In 2000, Palmer \& Meyer demonstrated that motor movement in performance is controlled by more abstract conceptual thought as skill increases, rather than being controlled by plans to effect specific movements. Adult and child pianists were tested on transfer tests where either the motor actions or conceptual concepts of the music were similar or different (Palmer \& Meyer, 2000). Skilled adults transferred better when the conceptual ideas of the transfer test were the same, no matter if motor conditions were similar or not (2000). Children at intermediate levels could make transfer for both motor and conceptual similarities, but inexperienced children could only transfer if motor and conceptual ideas were exactly the same (2000).

This was the first test to show that abstraction in motor motion happens in complex music performance skills. The results suggest that action plans are independent of the actual motor movement when pianists are skilled (Palmer \& Meyer, 2000). This result seems to be related to Duke et al. (2011), who showed that performance is optimized when players purposefully direct their focus of attention to produced sound rather than the motions themselves.

There is a clear trade-off in music performance between speed and accuracy. Musicians continually search for a "sweet spot" in fast tempi where speed is high enough to achieve the musical goal, but accuracy and control are not sacrificed or endangered. In 2007, Pfordresher et al. designed a study that models this speed-accuracy trade-off for sequential motions that are timed, as in music performance. The model was based on the idea that each event, as it happens, is retrieved based on the events that surround it, which may provide context (Pfordresher et al., 2007). Pianists tested the model on a passage using eight different tempi.

From this study, one can learn more about why skilled musicians are able to perform at very fast speeds with little or no error. The study found that "errors in production originate in the incremental retrieval of events over time. Thus, serial order and accuracy may both share a common source: the time over which the contextual representation of a sequence is updated [in working memory] during production" (Pfordresher et al., 2007, p. 92).

Results showed that working memory was involved in properly retrieving events in time-order (Pfordresher et al., 2007). This can only be controlled by a pianist through attention, yet pianists may assume the benefit of having enhanced working memory as musicians. Accuracy, however, was found to be related to one's motor-dexterity and overall skill as a pianist (Pfordresher et al., 2007), abilities which can continually be improved.

In 2018, Furuya et al. developed and proposed a new system for monitoring sensorimotor feedback during piano performance. The system was designed to observe and to monitor skilled pianists 
in order to learn from their responses to feedback. It included a non-invasive brain stimulation system that can assess cortical activity for auditory-motor integration while a pianist is playing (2018).

Secondly, a system was created to manipulate the weight of a key dynamically so that changes the pianist makes due to feedback from weight changes could be monitored in real-time. Therefore, the system can monitor both auditory feedback and proprioceptive feedback (Furuya et al., 2018).

This particular study provided interesting results about piano playing with normal feedback vs. playing with white noise feedback. Hearing normal piano tones while playing demonstrated excitability in the motor cortex area for the monitored index finger, only and exactly prior to that finger's next movement (Furuya et al., 2018). This is the first study to show that auditory feedback and its relationship to motor cortex excitability is time dependent (Furuya et al., 2018). Also, this same excitability effect did not occur during white noise feedback; therefore, correct feedback in performance was shown to be crucial to continuing to make correct and optimal sequenced events (Furuya et al., 2018).

In the future, this new system may enable us to know more about how computation in the brain manipulates sensory feedback and how it interacts with sensorimotor control ("computation"). Furuya reports that, "our new robotic device attachable to a musical instrument can further infer computational processes underlying integration of sensory feedback into motor adaptation during musical performance" (Furuya et al., 2018, p. 218).

In 1997, Palmer reported that motor systems are thought to use internal clocks that act as timekeepers to control how long it takes to make a movement's trajectory (1997). Timekeepers also create an abstract concept of the musical beat so that one knows when future movements should happen, and generally, they operate at whatever beat level one perceives to be most salient (Palmer, 1997, p. 128). Their primary function is now understood to monitor complex timing events, like motions happening between two hands or between multiple performers. Timekeepers acting within the motor system during motor execution may be responsible for expressive timing that is purposefully out of pace with the internal clock.

“Further tests of solo piano performances [in the 1980's] indicated that timing was directly controlled at the beat level just above the level of individual notes, which allowed the two hands some temporal independence in coordinating note events below the beat level that differed in duration" (Palmer, 1997, p. 130). Thus, in the Prelude no. 3 in C\# from WTCI the controlling beat level would be the dotted quartet note; the temporal independence can be seen in the possibility of the two hands 
attacking the final eighth note of a measure at slightly different times. There is also evidence that each of a pianist's hands has its own timekeeper, allowing for this flexibility.

Findings by (Schaffer, 1981; Schaffer, 1984, as cited in Palmer, 1997, p. 130) "suggest that temporal precision in performance is influenced by the structure of the sequence-in particular, the salience of the beat level or tactus." There is also evidence that the time it takes to play an entire piece is monitored and understood as well, and that this ability may be related to one's ability to remember tempo (1997).

When an overall performance tempo changes, studies have suggested that this does "affect the perception of duration patterns. If performers use expressive timing to bring about a desired structural organization for a particular tempo, different perceptions might result for the same relative expressive timing pattern played at a different tempo" (Palmer, 1997, p. 131). This would mean that the generalized motor program involved in performance is not simply changing tempo as one of its parameters at a straight rate; instead, it would require the ability to be flexible (1997).

\section{Thought in Performance}

In 1989, Palmer performed a study to learn how pianists employ expressive timing. Can a pianist's thought be mapped to a performance?

Palmer found that there were three categories of common expressions that pianists use: chord asynchronies, pauses or rubato at phrase boundaries, and the application of legato and staccato, which is scientifically equivalent to the timing onsets and offsets of notes. Palmer refers to onsets and offsets as "overlap patterns."

All these methods were observed to decrease when participants were asked to play unmusically, and in turn, each increased when participants were asked to exaggerate (Palmer, 1989, p. 344). Secondly, the performers used their own individual interpretations of the structure of the piece to decide how timing methods would be performed. All of these interpretations could be correlated to individual performers' written notes on their scores (Palmer, 1989, p. 344). A different interpretation did not musically work with another performer's expressive timings. Instead, when expression somehow accents structure, a performer's concept of the piece is brought to light (Palmer, 1989).

Chord asynchrony was used to highlight a melody by playing the melody notes prior to other accompanying chordal notes, giving it prominence. This study showed that pianists knew they were playing a melody louder than its accompaniment, but they did not know that they were sometimes 
playing it sooner (Palmer, 1989, p. 345). Similarly, past studies have shown that pianists sometimes are not sure if they are executing accents via rhythm or loudness (Palmer, 1989, p. 345).

Palmer explains that motor programs have both high- and low-level components. Higher-level components may include conscious intentions and emotional components. Lower-level components include automaticity and parameters that extend GMPs, which are not necessarily available to conscious awareness. Therefore, plans are carried out partly consciously, and partly not (Palmer, 1989, p. 332). Secondly, rubato patterns were employed most at phrase boundaries, usually to slow phrase endings so as to mark the beginning of a new phrase with a "quasi a tempo" (Palmer, 1989, p. 332). Most pianists would be aware that they are doing this.

In studying overlap patterns, the relationships between the ending of a note and the next onset were found to be proportional to the durations of both the preceding and following note (Palmer, 1989, p. 345). Therefore, planning for timing must be made far enough in advance for its correct execution to be proportionally correct.

In summary, this research suggests that "pianists share a common set of expressive timing methods for translating musical intentions into sounded performance" (Palmer, 1989, p. 344); yet each expressive choice is uniquely tied to a performer's concept of the musical structure. In this way, a pianist's thoughts are mapped into performance. 


\section{Conclusions and Thoughts on Future Research}

Pianists have a significant opportunity to benefit from current neuroscience research on learning, memorizing, and performing music. While some research findings may simply confirm knowledge that musicians already possess, there is value in having scientific confirmation. Research that clarifies, rebalances, or introduces new concepts for optimizing musicianship is invaluable.

Procedurally, current research suggests that one should listen to a full performance first, analyze the music's structure, and use these devices to create an auditory image of the desired goal in one's mind. Using the auditory image as a guide, one should then use many kinds of imagery, knowledge of the structure, and organized physical practice in learning. Practice should always be goal-oriented, and continually evaluated for progress. Having a goal, being constantly aware of it, and fully paying attention may matter more in preparation for piano performance than anyone once believed.

It seems likely that first modes of learning are most deeply related to one's own strengths. As long as multimodal methods are employed early, alongside an auditory image, the original mode of practice will likely be shown more definitively to be insignificant.

Strong practice habits embrace desirable difficulties, including variable practice for motor skill strength, interleaving to create contextual interference, spacing the timing of practice for optimal memory consolidation, and recall strategies that optimize testing effects and seamless coordination with associative cuing. These practice techniques are essential, even when they detract from early performance gains.

Practice that connects different modes of memory, such as hearing and spatial skills (practicing while looking at hands or imagining one's physical body), or motor skill coordinated with emotion (practicing performance cues), strengthens multimodal memory connections. Increasing cognitive complexity and multimodality in practice creates more synaptic connections and builds wider, stronger networks of memory interconnections for memory strength and long-term learning.

Repetition is still important. Motions must be allowed to become automatic and overlearning is highly beneficial. Continued practice when gains seem to be incremental increases one's mental representation of music in the brain. However, when one is tired, it may be more useful to take a break or a short nap. Overall, expertise in piano music is the most important factor for how well one can memorize and perform piano music. Pianists should spend time, not only practicing, but also studying and listening to music. 
Musicians can be grateful that their training enhances working memory function. However, attention guides working memory. Practice that changes the focus of attention allows for flexibility and creates confidence that, if one is distracted while playing, one's performance need not be derailed. Similarly, paying attention to varying aspects of the music allows a mental representation to be as strong as possible and provides ample opportunity for making the best musical choices for attention in performance.

Music is hierarchical-and so is our brain function for music. Nearly everything musicians do is tied to the structure of the music: initial perception, practicing, phrasing choices, expressive timing, tempo choices, and understanding of form. The hierarchical constructs of the music are inextricably tied to how the brain memorizes and retrieves that information in performance. Musicians must know the structure of a piece and which hierarchical levels are deeper than others, in order to create convincing musical goals, to memorize, and to perform well.

During performance, memory systems may be trusted to update one's sense of timing and to help in executing plans for expression. Research on planning during performance strongly suggests that one should concentrate only on the current sound that is being made and the planning of the next one, not on the physical motions required-and never on the past. Because an entire phrase can be kept in working memory at once, it seems likely that planning for the next phrase will be shown to be the largest future event one can plan during a performance.

Focusing on the mental image, the desired sound one wants, is far more optimal than focusing on motor motions, themselves. One should hear the auditory goal and then allow it to happen as simultaneously as possible. Perhaps one way to minimize dualism in performance is by practicing multimodal imagery with the singular intention of creating the currently desired sound. This would place the auditory imagery and its action as tightly together as possible and facilitate their happening fluently in actual performance. In the future, perhaps research in music embodiment or multimodal learning processes can lead us beyond a place of dualism, into a heightened awareness of "thinking-doing," or "anticipating-acting" in a more holistic way.

It seems likely that memory consolidation research will soon lead to more specific guidance for practice. Perhaps it will be able to include specific variables. For example, maybe we could know that for practicing a piece with a scaled difficulty of $8 / 10$, if the piece is brand new, and if we have 2 hours to spend practicing, that we should take a first practice break in about 25 minutes time. Until more is known, pianists can place more trust in taking breaks and getting enough sleep to support their work, knowing that the brain is providing extra energy toward the hardest parts of what they do. 
Perhaps future research in spaced practice and memory consolidation might incorporate music of higher difficulty and greater length. If feature binding for an entire piece takes significantly more time than feature binding for one musical event, perhaps break intervals for memory consolidation when learning a Brahms concerto might be different from those for a short, one-handed melody such as have been used in many studies. This is an area to watch for future results.

It would be helpful to know more detail about how style schemas operate, because pianists' errors have been tied to musical textures where performers are simply not paying enough attention. This is often caused by habituation, tied both to experience and also to style schemas, because mistakes tend to happen in musical textures that one "already understands" or where one directs the least attention.

Pianists might consider being more proactive in considering textures in an opposite way, or a different way than per usual. For instance, noticing inner voice leading in chordal structures, or noticing the melodic contour of a tenor voice in a different register, or from a differently segmented place, may bring more clarity to those areas. Knowing more specific practice methods that provide proper attention to everything may help to reduce common errors that now seem as though they are rather predictable.

Because some past studies have shown that labelling notes and attempting to consider chord names when memorizing difficult musical styles was helpful, perhaps future research may help with parsing or other strategies for memorizing modern music with structure that is less hierarchical or predictable.

Perhaps research can specify more about how clear a musical image should be in one's mind prior to the start of physical or multimodal practice. Alexander Technique research suggests that difficulty in creating a completely clear auditory image prior to physical practice is detrimental, because it then requires significant effort and time to acquire. If multimodal practice is optimal for strong memory representations, and auditory and motor systems are strongly coupled in musicians, perhaps one cannot achieve an optimal auditory image until other forms of practice, especially physical practice, have ensued.

While it is fascinating that individual differences in learning and propensity for music are being scientifically demonstrated, many questions still remain concerning individual differences, their sources, and how they operate in real-world situations. Since how much one cares about music is a factor in learning, perhaps future research can determine if there are limits to the amount of expertise one can attain, or if training or the choice to continue long-term learning may affect learning or its speed, even when predisposition is not present. 
I once had a chamber-music coach, a well-known pianist in the Pittsburgh area, whose mantra was, "I can never tell you how much time I have wasted in my life practicing." Instead, it might soon be possible to tailor specific paths for training that are optimal for each individual's strengths and weaknesses, as well as clear, optimal paths for professional pianists to employ. Future research has the potential to optimize music pedagogy, preparation, memorization, and performance, perhaps in the foreseeable future.

Future research is likely to uncover cognitive ability that is greater than the realm of music, especially because research often uses the field of music to learn about the larger human condition. Similarities and overlapping brain function between auditory and verbal streaming and perception may still have a lot to teach us. The strength and tightness of networks between sensory modalities is a rich and complex topic for research, still so much in infancy that it is difficult to predict what may be revealed or what its ramifications might be.

Palmer reminds us that music "offers a well-defined domain in which to study basic psychological constructs underlying sequence production, skill acquisition, individual differences, and emotional response, all of which will be the focus of future research directions" (Palmer, 1997, p. 134). 


\section{Appendix A - Glossary}

Acquisition/Skill Acquisition. The gaining or learning of a new physical ability, such as learning a new piece of piano music. Plasticity allows the brain to "rewire" and reorganize itself in order to change and adapt, allowing the brain to learn a new memory or skill. Changes in the brain during the acquisition of music and how these changes take place efficiently are actively being studied.

Association. A process by which memories become connected, such that the activation of one memory may cue another. Associated memories form a context for each other. Association often occurs between memories of events close to each other in space, time, or both. More complex, metaphorical associations may be formed between memories correlated in other ways. Association is a primary organizational feature of human memory (Snyder, 2000).

Associative cuing. When processing chunked information, the last element in a chunk may act as a recall cue for the next chunk. People seem to remember long, time-ordered sequences of information by the process of cuing. Cuing chunks in sequence may create long chains of associative cues. This is one method of recalling a long piece of music in its correct order (Snyder, 2000, p. 55) (Chaffin et al, 2016).

Attention. The "limited mental energy. . . that powers the mental system, . . . the stuff that gets focused when we pay attention" (Ashcraft \& Klein, in Levitin, 2011, p. 322). One deliberately chooses to use one's concentration for a particular activity while paying attention.

Auditory dorsal stream. Currently, it is believed that the dorsal (higher) stream, in the dPMC (the dorsal Pre-Motor Cortex) carries spatial information and is related to taking an action. It guides auditory motor actions, by recognizing patterns of how things are happening in the music and making decisions about producing a motor motion. The dorsal auditory stream is known as the "how-do" portion of a dual stream model of auditory processing where the auditory ventral stream is the second portion of the dual model (Baumann, 2007).

Auditory ventral stream. The auditory ventral (lower) stream, in the vPMC (ventral Pre-Motor Cortex), is thought to process the meaning of sound and concepts; it identifies events, and processes any information that tells the brain "what" it has heard. The auditory ventral stream is the "what" portion of a dual-stream model of auditory processing where the auditory dorsal stream is the second portion of the dual model (Baumann, 2007).

Audiation. A complete internalization of music that includes the understanding of musical meaning. The term was coined in 1975 by Edwin E. Gordon, who developed a system for music education that optimizes internalization. The term is now often used to refer to hearing music in one's "inner ear" while reading a score (Gordon, 2012).

Auditory events. See event.

Auditory imagery. Auditory imagery is any mental representation of music in one's mind (Simmons, 2007). It is used by musicians and non-musicians alike, but has been shown to be multimodal (Keller, 2012) and important for successful piano performance from memory (Highben \& Palmer, 2004). 
Auditory Scene Analysis. Auditory scene analysis is the study of how we take multiple auditory inputs from a complex environment and determine what we are hearing. Auditory scene analysis is mostly about determining which sounds came from the same source. (Snyder, 2000). Also see streaming.

Auditory working memory/Tonal working memory. Working memory refers to a cognitive system where information is temporarily stored with a limited capacity that allows for online manipulation of the maintained information. Auditory working memory stores both phonological information that forms verbal representations such as words and phonemes, and also non-phonological information such as pitch, speech prosody, and timbre. The non-phonological information is referred to as tonal working memory. Tonal working memory concerns the maintenance and the online manipulation of tonal information (Schulze \& Koelsch, 2012).

Bilateral. On both sides of the brain, as one thinks of left and right sides of the head.

(BOLD) Blood-oxygenation-level-dependent response/BOLD response. This response is revealed with fMRI by measuring magnetic properties of blood flow and by showing the differences in magnetism between deoxygenated blood and oxygenated blood. A BOLD response measures functional connectivity and network properties of the brain. (Herholz \& Zatorre, 2012). Also see Functional magnetic resonance imaging. fMRI.

Bottom-up processing. Information processing at the perceptual level. Unlike top-down processing, bottom-up processing operates primarily on perceptual input and usually does not involve memory. (Snyder, 2000).

Boundaries. Memory influences how we perceive groups of events, when they end and other groups of events begin. Boundaries in time are endpoints that our memory systems perceive as separating groups and patterns of information (Snyder, 2000).

Category. In cognitive psychology, a grouping together of features of experience or memory. Categories are collections of perceptual representations or concepts that seem somehow related. Categories may be perceptual or conceptual. Some perceptual categories are innate, whereas other perceptual and most conceptual categories are learned. A category has a central or prototypical member. Scale tones and intervals are examples of melodic categories (Snyder, 2000).

Caudal. Back or posterior; a term used to locate parts of the brain.

Chunking. A way of reducing short-term memory load by coding at a higher level. Chunks are small groups of elements (5-9) that, by being frequently associated with each other, form higher-level units, which themselves become elements in memory. An example of chunking might be memorizing a telephone number by converting it from single to double digits, from two-eight-one, one-four-eightfour, say, to two-eighty-one, fourteen-eighty-four. Chunking is essential to the hierarchical organization of information, which makes its mental representation in memory much easier and more efficient (Snyder, 2000). 
Closure. The tendency of perceptual groupings to have beginning and ending boundaries. Closure tends to occur at points where changes in degree of similarity or proximity occur. That is, boundaries tend to be created where new kinds of events occur or where the distance in time or pitch interval between events changes. Closure may be partial; establishing degrees of closure is essential to establishing hierarchies, or multiple levels of organization in music and language (Snyder, 2000).

Cochlear nerve. Another name for the auditory nerve.

Cognitive load (Also known as attentional load). The amount of attention that can be given to any one task at once or the quantity of things that can be given our attention at the same time. This may also be called attentional load or the capacity of our focus of attention (FOA) (Ashcraft \& Klein, in Levitin, 2011).

Constructionist theory. This theory holds that memory's prime purpose is to anticipate the future. Instead of a record-by-record account of every experience, new experiences initiate changes in one's memory systems as one continually learns and adapts to their environment. The brain remembers abstractions or generalizations about its environment and one remembers those structural rules, but not details. Therefore, remembering is based on reconstructing the past, based on our generalized knowledge and one's current experience, in concert with the contents and workings of our memory systems (Levitin, 2011).

Context. In cognitive psychology, a web of associations connected with a particular memory. When a particular memory is recalled, much of its associated context is primed for recall as well. Just as a particular memory can cause the priming of a context, so a rich context can provide many alternative pathways to recall of that particular memory. Not all contexts are conscious, however: unconscious contexts, which include schemas, can guide consciousness without ever entering awareness themselves. (Snyder, 2000).

Contextual interference. A "learning schedule in which a task and its variations are interweaved. The idea is that injecting contextual interference by frequently alternating motor tasks results in increased long-term learning that is more likely to transfer to new tasks" (Caramiaux et al., 2018). Purposeful interference, or good interference, is an aid to long-term learning (Bjork, 2018).

Contour. In music, a particular and characteristic pattern of upward and downward melodic motion, which, following the spatial metaphor of melody, is described as though it were visual. Contour is one of the pattern aspects of melody; under some conditions, melodies can be recognized by their contour alone. Contour can also be applied to rhythms, describing their movement through longer and shorter durations or time intervals (Snyder, 2000).

Continuity. The concept that every musical event either develops a connection with the previous event or separates itself from it to some degree. Continuity is one of the primary grouping factors in music (Snyder, 2000).

Cuing. Connections are created and facilitated between groups of simultaneously activated neurons. A neural event that activates an associated long-term memory may also activate another long-term memory associated with it, and so on. This process is called cuing (Snyder, 2000). 
Divided attention. When learning is distracted by more than one focus of attention. Divided attention also happens when one must monitor several streams of information at once, perhaps from several spatial locations, i.e., listening for one bird call in a busy forest. Divided attention taxes our resources, because attentional capacity is limited (Middlebrooks et al., 2017).

Dorsal. North or superior; the term is used to locate parts of the brain.

Early Processing. Provides our immediate experience of the present moment of music in the focus of awareness (Snyder, 2000). Also see event fusion.

Early Right Anterior Negativity (ERAN). Early right anterior negativity is an automatic response in the brain that takes place when violations of complex auditory sequences are recognized, based on violations of the Western tonal system, which might be noticed in melodies or in common chord progressions (Palmer \& Meyer, 2000).

Echoic Memory. The first stage of auditory memory, echoic memory is a sensory memory of large capacity that decays rapidly, like an echo. Information persisting as echoic memory is in the form of raw sensory data that are not coded or conceptualized yet. Its function is to allow sensory data to persist long enough so that coding and recognition can take place (Snyder, 2000).

Electroencephalogram (EEG). The electroencephalogram detects when and how often neurons are firing, because they produce small electric currents when firing that can be measured. Electrodes are painlessly placed on the surface of the scalp. Because the firing of one single neuron only produces a weak electric signal, EEG can only detect when large groups of neurons are firing in synchrony. An EEG is very sensitive to timing, with resolution within one millisecond; however, an EEG provides poor spatial resolution. Because music is time-based, EEG has the best temporal resolution of all of the tests now used (Levitin, 2007).

Encoding. The creation of long-term memories. Permanent electrical and neurophysical changes take place in neurons, the nerve cells of the brain, that permanently strengthen their connections. Encoding creates what we think of as a memory (Snyder, 2000).

Episodic memory. A type of long-term memory for specific events, in a specific time order, and in relation to the self. Episodic memories are autobiographical, whereas general knowledge, another type of long-term memory, is not. Episodic memory records events as they happen to us. However, because remembering is itself an event, episodic memories are copied when they are recollected and the copy replaces the original. This makes our episodic memories vulnerable to various kinds of transformations and distortions, especially through their interaction with our semantic memory categories and schemas. In a piece of music, episodic memories would be of the details of the sound of particular passages of music and their time order (Snyder, 2000). Also see semantic memory.

Expectations. Memory influences how we decide when events or groups begin and end, and how we comprehend time sequences of overall events, giving us the ability to predict or anticipate what might come next. Context memory and schemas support our expectations (Snyder, 2000). 
Explicit Memory. The kind of memory used when we consciously memorize or recall information. It is memory of events or concepts that can be described in words. It requires conscious effort to use or recall it (Snyder, 2000).

Event. In cognitive psychology, the perception that something has happened, caused by a detectable change in the environment. Acoustical events are the fundamental perceptual units of music, from which all musical groupings, phrases, sections, and the like are built up (Snyder, 2000).

Event fusion. Acoustical vibrations that occur closer together than 20 events per second fuse together to form pitches, individual events, boundaries, changes in pitch, changes in loudness. This information processing happens early, between sensory perceptions in the ear to the auditory cortex. These are the result of limits on the speed at which these neurons can process incoming information (Snyder, 2000).

Event-related potentials (ERPs). Derived from electroencephalographic (EEG) measurement of neural activity. An event-related potential measures voltage changes in the brain that follow, or precede, the onset of specific visual, auditory, or other sensory stimuli, as well as cues signaling motor preparation, motor execution, or covert mental operations (e.g., imagery). The ERP is obtained by extracting and then averaging multiple temporal segments from the ongoing EEG that define the event of interest. The amplitude, latency, and topography of the resulting positive and negative deflections are taken to index the underlying mental operations. ERPs elucidate the cognitive processes in perception, attention, emotion, action, memory, and more (Bradley \& Keil, 2021).

Explicit memory. A type of long-term memory whose contents are available to consciousness, in contrast to implicit memory, another type of long-term memory, whose contents are not. Explicit memory can be further divided into episodic memory and semantic memory, also referred to as "autobiographical memory" and "general knowledge," respectively (Snyder, 2000).

Feature extraction. A basic process of perception. This happens very early in the perception process, during the persistence of echoic memory. Different features of sounds are believed to be extracted by various special-purpose feature detectors. These features are then "bound" into units that represent objects or events (perceptual categorization). This process must happen before higher-level coding and recognition can take place. Long-term memories are often referred to as bundles of features (Snyder, 2000).

Feedback loop. In information processing, a loop connecting the output of a process back into its input. Examples of this are the short-term memory rehearsal process, the connections between long-term memory and feature extraction, and the connections between long-term and short-term memory (Snyder, 2000).

Feedforward control. Feedforward control is a method by which the pre-motor and motor cortex prepare to make movements based on sensory input (Brown \& Penhune, 2018).

Focus of conscious awareness. (FOA) (Also known as Focus of attention). The part of our experience that is happening right now, the focus of conscious awareness (or just the focus of awareness FOA), usually consists of a vivid sensory aspect and an activated memory aspect. Its capacity is one to three items at most. The focus of consciousness is different from short-term memory. Items that are currently unrehearsed can be present in short-term memory, but are not in consciousness (Snyder, 2000). 
Form. In music, any grouping longer than $3-5 \mathrm{sec}$, the time limit of short-term memory, to include all kinds of sections, movements, and whole pieces. The significance of the concept of musical form is that it cannot be perceived directly, unlike events, groupings, and phrases, but must be built up from representations in long-term memory. Formal (section-length) proportion and long-term thematic associations must be established by comparing different materials through long-term memory; these become clearer as long-term memory strength increases with repeated listening. Indeed, a large part of the recognition of musical form depends on repeated listening (Snyder, 2000).

Functional Magnetic Resonance Imaging (fMRI). Functional magnetic resonance imaging operates because the hemoglobin of the blood is slightly magnetic. A fMRI machine is a giant electromagnet that shows differences in magnetic properties in blood flowing through the body at any point in time. It traces changes in blood flow. Neurons also need oxygen to survive, so the blood carries oxygenated hemoglobin to neurons, allowing blood flow to the brain to be trackable. Actively firing neurons need more oxygen than those at rest, so brain areas that are busy with cognitive tasks will have the most blood flow at any given time. This kind of MRI is called functional because it can be used while the brain is thinking or functioning. (Levitin, 2007, pp. 128-9) fMRI provides good spatial resolution when examining cortical and subcortical structures (Herholz \& Zatorre, 2012). Also see BOLD response.

Generalized Motor Programs (GMPs). A motor program represents the fixed relationships between individual movement segments that comprise a motor skill. They are invariant structural patterns of proportional relationships among movements that can be executed under different performance parameters (e.g., performing the rhythm of a melody at various tempi) (Simmons, 2007).

Gist. A general model of what happened in a particular place at a particular time, generally in relation to a fixed set of expectations, or schema, and one of the ways our semantic memory categories and schemas interact with episodic memory. Most often, we do not remember exact details of situations, but only the gist of what went on. Exactly what constitutes a musical gist remains an open question (Snyder, 2000).

Grouping. In cognitive psychology, the tendency for individual elements in perception to seem related and to bond together into units; the result of such a process. Grouping may be either innate or learned. Innate grouping appears to happen automatically in perception and occurs without any contribution from long-term memory, whereas learned grouping requires the use of long-term memory. Grouping can occur on multiple levels (Snyder, 2000).

Habituation. An adaptation response as a result of neurons being stimulated repeatedly with an identical stimulus; their output of impulses does not remain constant, but decreases over time. Found in every kind of organism with a nervous system; truly one of the places where memory and perception become indistinguishable (Snyder, 2000).

Hierarchy. In cognitive psychology, a multileveled structuring of associations in long-term memory, where each higher level includes all the lower ones. Hierarchical memory structuring facilitates moving through large bodies of knowledge stored in the form of small chunks (Snyder, 2000). 
Image (a memory trace). Encoding happens in long-term memory when permanent electrical and neurophysical changes take place in neurons, the nerve cells of the brain, that permanently strengthen their connections. In this way, encoding creates what we think of as a memory. A memory is sometimes referred to as an image or a memory trace (Snyder, 2000).

Implicit memory. A type of long-term memory whose contents are not available to consciousness. Implicit memories include, most notably, memories of physical skills and memories for distributions of things. Emotional memories may also be implicit. Unlike other kinds of long-term memories, implicit memories are established slowly, with repeated practice, and are often not easily modified. Playing a musical instrument may involve both explicit and implicit memory. For example, reading a particular notated pitch on a page and knowing its fingering on an instrument involve explicit memory, whereas correctly performing all of the other physical actions required to produce that note reliably involves implicit memory (Snyder, 2000).

Individual Differences. Individual differences refer to unique qualities and abilities in each person that may differ from standardized norms (Guenther, in Levitin, 2011).

Interference. In cognitive psychology, an effect that can occur between long-term memories that are similar. Similar memories can confuse association, interfering with the cuing process and creating irrelevant pathways that hinder recall (Snyder, 2000).

Lateral. Horizontal in direction, going from core of the brain toward outside of brain.

Long-Term Memory (LTM). A very large, possibly permanent memory, whose contents are usually unconscious. Meaning and understanding are derived by processing present perception through longterm memory. Long-term memory is often divided into explicit memory (available to consciousness) and implicit memory (not available to consciousness). Explicit long-term memory is further divided into semantic and episodic memory. Long-term memory is essential to the comprehension of large-scale musical form (Snyder, 2000).

M1. The primary motor cortex.

Magnetoencephalography (MEG). Detects functional, evoked sensory responses, and can measure synchronization of neural activity in oscillations. It provides good temporal resolution and acceptable spatial resolution for cortical sources. This is a relatively direct measure of neural activity (Herholz \& Zatorre, 2012).

Medial. Toward the middle core of the brain.

Memory consolidation. The process that modifies the neural representation, or memory, for a new skill in ways that affect performance when skills are recalled. It occurs in two stages: consolidation-based stabilization (stabilization) and consolidation-based enhancement (enhancement) (Simmons, 2007).

Memory stabilization. Makes new memories resistant to interference and sustains levels of performance achieved by the end of initial practice. This phase of memory consolidation happens during waking hours and continues for 4-6 hours after the end of practice (Simmons, 2007). 
Memory enhancement. The second phase of memory consolidation; this phase modifies memories in ways that enhance performance when skills are recalled. This phase of consolidation most often relies on neural processes that occur during sleep (Simmons, 2007).

Memory trace (an image) Encoding happens in long-term memory when permanent electrical and neurophysical changes take place in neurons, the nerve cells of the brain, that permanently strengthen their connections. In this way, encoding creates what we think of as a memory. A memory is sometimes referred to as a memory trace or an image (Snyder, 2000).

Mere exposure effect. The effect that people tend to like something better if they have simply been exposed to it in the past. This research finding was referenced by Stalinski \& Schellenberg (2013).

Mismatch-Negativity (MMN). Mismatch negativity is an automatic brain response to deviant stimuli. It can be evoked when attention is directed away from normal, expected, or current auditory stimuli. MMN has component parts that occur later in time than when the deviant stimulus takes place, e.g., P3a and P3b, which can be detected. They reflect the orientation of attention towards the deviant sound, and are evoked when one detects and evaluates a deviant target (Palmer \& Meyer, 2000).

Motor schemas. A schema for motor skills stores memories of a particular motion one has made, and also abstracts rules about that type of movement. A set of these rules comprises the motor schema, which can be called upon later and can be modified for specific situations. Schemas allow us to produce movements we have never made before, because they are related to movements we have already made, and because we are able to produce expectations about how to enact the new, related movement (Schmidt, 1975).

Recall Schema. Varied practice generates a set of rules, called a recall schema, that facilitates the execution of a learned skill in novel contexts while retaining the integrity of the original governing generalized motor program (GMP) (Simmons, 2007).

Multiple-trace memory theories. A relatively new class of memory-system models which contends that each experience we have is preserved with high fidelity in our long-term memory system. In retrieving a memory, interference may come from other traces that are competing with our attention - if they have slightly different details - or if some of the details of the original memory trace have degraded over time. In these theories, abstractions of information about our memories are also retained; current theories vary (Levitin, 2007).

Musical culture. An aggregate of evolving musical concepts and practices shared by a group of individuals. Through schemas and conceptual categories (contexts) formed in the minds of those individuals, musical culture structures expectations about many aspects of music. Also see context; schema (Snyder, 2000).

Musical lexicon. All the representations of the specific musical phrases to which one has been exposed during one's lifetime (Peretz et al., 2019).

Neuron. The primary cells of the brain, also located in the spinal cord and the periphery of the nervous system (Levitin, 2007). 
Neurotransmitters. Chemicals that travel throughout the brain and bind to receptors attached to other neurons. If a receptor is like a lock on a neuron, a neurotransmitter is like a key. It swims across a synapse to a nearby neuron, causing the neuron to fire or preventing the neuron from firing (Levitin, 2007).

Nuances. In music, the changes in musical parameters that take place within a category; for example, nuances may be small fluctuations in pitch which do not result in a pitch being perceived as belonging to a different pitch category. Nuances, because they exist within a category, are much harder to remember than the categories themselves. Because they exist within a category, nuances are not part of syntax, that is, they do not contribute to pattern differentiation. Nuances do frequently represent emotional or expressive information (Snyder, 2000).

Overlearning. Studying or practicing more than what is needed to master the target material (Soderstrom \& Bjork, 2015).

Overt/Covert. Repetition of information in short-term memory may be aloud (overt), or under one's breath (covert) (Schulze \& Koelsch, 2012).

Perceptual binding (in Short-Term Memory). A psychological process whereby the elementary features of perception are bound together into objects and events (Snyder, 2000).

Phonological loop. The part of working memory that handles auditory and verbal information, both language and music. It has two parts: (1) Storage, the phonological store, where we keep information in STM for rehearsal, and (2) An articulation mechanism to maintain memory and strengthen it. If rehearsal continues enough, information goes to LTM (Schulze \& Koelsch, 2012).

Phonological Store. One of two parts of the phonological loop, it is a storage area for information that we keep in STM for rehearsal (Schulze \& Koelsch, 2012). Also see phonological loop.

Pitch Class. A set of frequencies of pitches that allow them to be called by the same musical letter name; all frequencies, for example, that are called B-flat.

Pitch/Pitch Height. The aspect of musical sounds that is correlated with frequency and gives them the quality of "highness" and "lowness," referred to as pitch height. To display this quality, a sound has to have a certain basic minimum of repetition in its microstructure. The opposite of pitch in this sense is a sound with no repetition in its microstructure, which is called noise. Differences in height between pitches are called pitch intervals. Different pitches that have equal differences in pitch height (the same interval) between them have their frequencies in the same ratio (Snyder, 2000).

Plasticity. The ability to create lasting changes in the structure or function of neurons or in brain organization in response to a stimulus, like an experience, or the acquisition of a new skill. Plasticity allows the brain to "rewire" and reorganize itself in order to change and adapt (Herholz \& Zatorre, 2012). 
Priming. A process whereby the recall of a particular memory causes the low-level activation of other associated memories (a context), without this process necessarily becoming conscious. Priming, which makes it more likely that some of those semi-activated memories will also be recalled, is a type of implicit memory (Snyder, 2000).

Procedural learning/Procedural memory. Memory that is roughly the same as a skill, or how to do something physically. Procedural memory is formed through laborious, repetitive practice. It only refines slowly and is difficult to change. It is automatically recalled without conscious effort, and is usually expressed by performing the skill, rather than by recollection or verbal description (Snyder, 2000).

Proprioceptive. Information that the brain processes about the environment or a task about to happen prior to movement. In particular, these involve stimuli that are connected to the position and movement of the body prior to a conscious movement or task (Schmidt, 1975).

Proximity. In cognitive psychology, the principle that groupings form from perceptual elements close to each other in time. The principle of proximity is believed to be innate (i.e., not learned) (Snyder, 2000).

Recall Schema. When a movement is made, four things are stored: Initial conditions, parameters for the current movement, sensory feedback, and an analysis of how successful the outcome was (Schmidt, 1975). After each movement within a motor schema happens, the schema for recall, the recall schema, becomes more informed about the relationships among its related movements and it becomes more skilled to enable the next instance of that generalized motor program (GMP) (Schmidt, 1975).

Recollection/recall. A kind of cuing when one intentionally tries to cue a memory. It is not automatic; it happens by choice (Snyder, 2000).

Recognition. In cognitive psychology, a process whereby something in current experience cues its own long-term memory (Snyder, 2000).

Record-keeping theory. Holds that memory is thought of as a storage area, much like a library, where memories are recorded and physically stored in a particular place to be revisited later. Memory is thought to preserve the past, and newly made memories fill new memory locations (Levitin, 2011).

Rehearsal. In cognitive psychology, the active maintenance of elements in short-term memory. Conscious rehearsal consists of repeating something to ourselves to remember it for a longer period of time. Rehearsal is the primary way of remembering small amounts of information by keeping them active in the short term (Snyder, 2000).

Reminding. A kind of cuing that happens automatically when we see or hear something that is familiar (Snyder, 2000).

Retention interval. A gap of time between practicing or learning a skill, and a time when the skill's viability is tested to see how much it has been retained.

Rhythmic grouping. In music, a grouping of events in time, whose boundaries are defined by a relatively large change in some parameter. Several such groupings may form a phrase (Snyder, 2000). 
Rostral. Front or anterior, term used to locate parts of the brain.

S1. The primary somatosensory cortex.

Schema. A particular set of associations in long-term memory. Schemas are sets of expectations about how things usually are. They generally apply to particular kinds of situations in time and space, enabling us to move through these situations without having to pay attention to every detail. Indeed, if details conform to our expectations, we do not notice them at all. Schemas form our expectations about many aspects of a piece of music, and our initial long-term memories (after a few hearings) will be primarily schematic, that is, not of specific details, but of the gist of the piece. Traditional musical forms are essentially preconstructed schemas, which can provide a sense of knowing where we are in a piece. Schemas describe what listeners bring to the musical experience. A musical culture could be defined as a particular collection of schemas (Snyder, 2000).

Semantic memory (Also known as declarative memory). A type of long-term memory for abstract categories, rules, principles, and the like, and not concerned with specific events or their time order as are episodic memories. Semantic memories seem to be related; not by their time order, but in conceptual hierarchies of various types, and seem not to be vulnerable to the same types of transformations or distortions that episodic memories are. Our general knowledge of music would consist of semantic memories (Snyder, 2000).

Selective attention. One's ability to choose a stimulus to attend to when there are multiple competing choices.

Short-Term Memory (STM). A type of temporary memory that persists for a short time (3-5 seconds on average; 10-12 seconds maximum) and whose capacity is limited to around 5-9 elements, or one "chunk." Items may be maintained for a longer time in short-term memory through the process of conscious rehearsal. Short-term memory length is one of the limitations on the size of small-scale groupings, and accounts for local musical structures such as phrases (Snyder, 2000).

Similarity. In cognitive psychology, the principle that groupings form from perceptual elements that are qualitatively similar. Some aspects of the principle of similarity seem to be innate (Snyder, 2000).

Sleep Consolidation. The second portion of memory consolidation that takes place during overnight sleep. See memory enhancement (Simmons, 2007).

Somatotopic. A point-for-point correspondence of a part of the body to a place in the central nervous system (McGill University, 2018).

Stable practice. Practice that utilizes similarities, that is, same tempo, or increasing tempi slightly in one direction. Unstable, or variable practice would vary tempi or goals more randomly, and utilize interleaving, imagery, or purposeful interference for longer-term learning (Simmons, 2007; Bjork, 2018).

Stream. A coherent grouping of acoustical events that is formed by their basic similarity and proximity to each other. When several streams of events occur simultaneously, we can only attend to one of them in detail at a time. The formation of streams is one of the basic ways in which we identify different sound sources in the environment (Snyder, 2000). 
Synapse. A gap between two nerve cells. Electrical impulses that travel through nerve cells are converted to a wave of chemical activity which travels across the synaptic gap. The specialized molecules that accomplish this conversion are similar in all organisms with nervous systems and are called neurotransmitters. It is currently believed that long-term memory consists of lasting chemical changes (referred to as "long-term potentiation") that occur at synapses and that alter the ability of the synapses to transmit neural pulses. The chemical changes that accomplish this take some time to become fixed, a process referred to as the "consolidation of memory." (Snyder, 2000).

Syntax. In this context, refers to sets of relations between identifiable patterns. This is a very general definition. It includes both traditions of rules for the use of particular kinds of functional patterns in particular musical styles and also relations between patterns that are unique to a particular piece. The essence of syntax as defined here is the ability to recognize and identify patterns as similar. This has been referred to as musical structure, as opposed to expressive nuance. Not all parameters of music seem to support these kinds of patterns. These proportional relations make it possible to form patterns that may be recognized and identified in different contexts. Only pitch, duration, and harmony are considered syntactical parameters (Snyder, 2000).

Subvocalization. Auditory imagery, the generation of internal sound, may coordinate with related movements in the articulatory system. Subvocalization refers to both auditory and kinesthetic portions of this process. It may be called silent singing, the inner voice, or singing in one's head. "It has been described as an intermediate step between thought and production of external sound." (Saintilan, 2015).

Tonal working memory/Auditory working memory. Working memory (WM) refers to a cognitive system where information is temporarily stored with a limited capacity that allows for online manipulation of the maintained information. Auditory working memory stores both phonological information that forms verbal representations such as words and phonemes, and non-phonological information such as pitch, speech prosody, and timbre. The latter is referred to as the tonal working memory. Tonal working memory concerns the maintenance and the online manipulation of tonal information (Schulze \& Koelsch, 2012).

Tonotopic. Organization of recognized characteristic frequencies (roughly equivalent to frequency) that is mapped in frequency order onto the basilar membrane and in the auditory cortex (The National Center for Biotechnology Information, 2001).

Top-down processing. Information processing that is primarily memory driven. Unlike bottom-up processing, which deals primarily with immediate perceptual experience, top-down processing involves the past. Most information processing in the nervous system is both bottom-up and top-down (Snyder, 2000).

Transcranial magnetic stimulation (TMS). Transcranial magnetic stimulation is a noninvasive procedure that uses magnetic fields to stimulate nerve cells in the brain. During an rTMS (repetitive TMS) session, an electromagnetic coil is placed against the scalp. The electromagnet painlessly delivers a repeated magnetic pulse that stimulates nerve cells in the brain (Hernandez-Ruiz, 2019). 
Transfer; Transfer of training. The influence of prior learning on new learning, either to enhance it or to hamper it. The ability and accuracy of the transfer, and the similarity between the original movement and the transfer movement, are used to determine how well a transfer takes place (American Psychological Association, 2020).

V1. The primary visual cortex.

Variable practice. In variable practice, skills are executed with different parameters each time, or often, placing higher cognitive demands on the pianist; these tasks exercise and enhance the abilities of a GMP Variable practice is necessary for developing recall schemas (Simmons, 2007, p. 43). Also see stable practice.

Ventral. South or inferior, term used to locate parts of the brain.

Working memory. The ensemble of memory components used in conscious thought. Working memory includes the focus of conscious awareness, visual and acoustical short-term memories, and a central executive component that maintains immediate plans and goals. As defined here, not all the contents of working memory are conscious. Currently unrehearsed elements in short-term memory, for example, are not conscious, but are part of working memory. Working memory shades off into the unconscious context that is currently semi-activated (Snyder, 2000). 


\section{Appendix B - Brain Anatomy}

\section{Overview}

According to the website, "A Brief Introduction to the Brain," (National Autonomous University of Mexico, 2013) the brain has four lobes that exist in mirrored fashion on the left and right sides of the head, commonly known as the left and right hemispheres (National Autonomous University of Mexico, 2013). The cerebellum and the four lobes comprise the human brain. Brain areas are divided into primary, secondary, and tertiary sensory or motor areas, due to the fact that some regions are more directly involved than others with sensory or motor processing (National Autonomous University of Mexico, 2013).

The so-called higher-order secondary and tertiary sensory and motor areas surround the brain's primary areas. Higher-order areas integrate information coming from the primary sensory areas, and they send complex information required for motor actions to the primary motor cortex. Some brain areas are labelled as supplementary, since they perform horizontal or coordinated functions with largerscale processing (National Autonomous University of Mexico, 2013).

Levitin (2007) states that the left hemisphere is required for naming and talking about aspects of music, for analyzation, musical syntax recognition, and for the use of the right hand or reading music from the right visual field (Levitin, 2007, p. 125). In his article, The Musical Brain: Myth and Science, Montinaro confirms that the left hemisphere is related to logic, while the right hemisphere is related to imagery and creativity, including the perception of timbre and melody (Montinaro, 2010, p. 443). Only the right hemisphere is active for music activity in non-musicians (Montinaro, 2010). Levitin reports that trained musicians demonstrate some activity in the left hemisphere, too, particularly when naming musical structure becomes involved (Levitin, 2007, p. 125). 


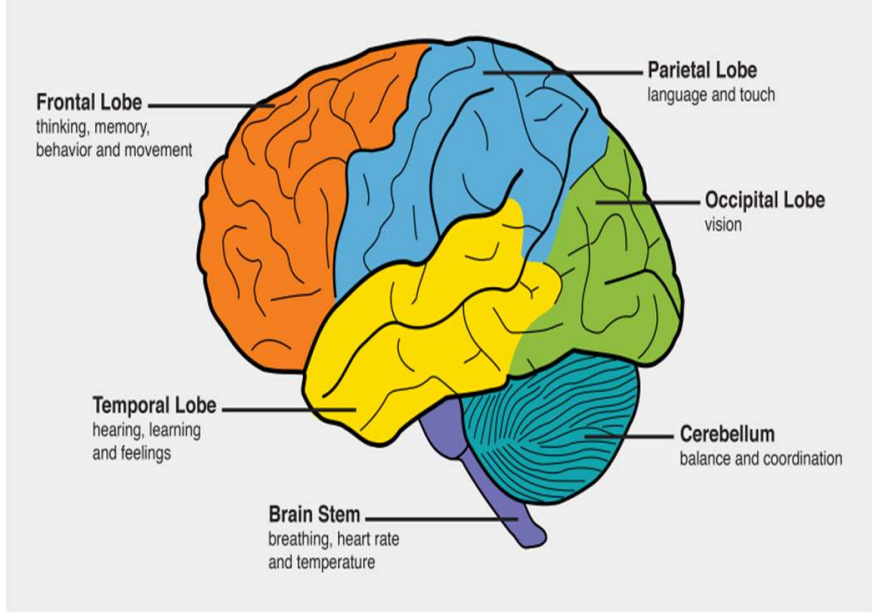

Figure 1. The four lobes of the brain and the cerebellum, as connected to the spinal cord via the brain stem.

(Brain injury E-learning resource, 2017).

Levitin (2007) explains that there are general responsibilities associated with each lobe. The frontal lobe is associated with planning, self-control, and the processing and making sense of our perceptions. The posterior part of the frontal lobe controls motor movements and spatial skills (Levitin, 2007, p. 88).

The occipital lobe processes vision. The temporal lobe is associated with hearing and memory. The cerebellum is evolutionarily the oldest part of our brain. It has long been known to control planning and timing of movement, and is also related to emotion (Levitin, 2007, p. 88 and pp. 174-5).

A McGill University website, "The Brain from Top to Bottom," (2018) explains that a portion of the brain's cortex is devoted to each of our senses. There is a visual cortex for vision, an auditory cortex for hearing, a motor cortex for movement, and a somatosensory cortex for tactile feedback and muscle movement (McGill University, 2018). The primary visual cortex, V1, is located in the occipital lobe. The primary auditory cortex, $\mathrm{A} 1$, is found in the temporal cortex on the sides of the head, around the temples just above the ears (McGill University, 2018).

The McGill website continues to explain that the primary somatosensory cortex, S1, maps exact parts of its area to exact body parts. It has a somatotopic organization; that is, there is a point-for-point correspondence of a part of the body to a place in the somatosensory cortex. Smaller body parts that require more fine control, like fingers, have larger areas of the somatosensory cortex, vs. i.e., the trunk, which has a smaller portion relating to coarser actions (McGill University, 2018).

The primary motor cortex, M1, in the posterior portion of the frontal lobe, is adjacent to the somatosensory cortex, in the anterior portion of the parietal lobe. It shares nerve fibers with sensory areas, and utilizes the same somatotopic organization, a quasi-minimalized map of the body (McGill 
University, 2018). The frontal cortices (FC) and prefrontal cortices (PFC) are areas covering the front part of the frontal lobe. They are also involved in motor movement (2018).

Levitin explains that brain activity works via its primary cells, its neurons (Levitin, 2007, p. 122). There are 100 billion neurons on average, each connected to between 1000 and 10,000 others. Levitin calculates that, for just six neurons, there are 32,768 possible connections. The brain has enormous power. Even with continued research, we are not likely to fully understand all of the brain's potential or what the implications of knowing might be (Levitin, 2007, p. 87).

Levitin shares that the brain can reorganize itself far more than was previously thought. This is known as brain plasticity. The brain can grow, change, and adapt (Levitin, 2007, p. 87). Reorganization and restructuring of the brain are the processes that create memory and learning (Simmons, 2007, p. 7). Music training has been shown to provide functional plasticity, changes that are required because of adaptation, training, and one's individual functions (Zatorre, 2013).

\section{Brain Areas for Memory Systems and Music Perception}

The National Center for Biotechnology Information provides details about the auditory system on its webpage, "The Auditory Cortex" (The National Center for Biotechnology, 2001). The primary auditory cortex, A1, receives all of the auditory information that originates at the ear. After the small bones of the inner ear receive sound-pressure vibrations, the cochlea, in the inner ear, analyzes the sound and arranges the perceived frequencies tonotopically, by pitch, along the basilar membrane. The information moves upward through the brain stem and is received by the primary auditory cortex (2001). The auditory cortex, A1, also has a tonotopic organization onto which this information is mapped. The auditory cortex then communicates with other areas of the brain to affect a meaning to the sound, emotion related to the sound, or to affect motion related to the sound (2001). This happens through various levels of memory processing, as explained in the body of this paper. 


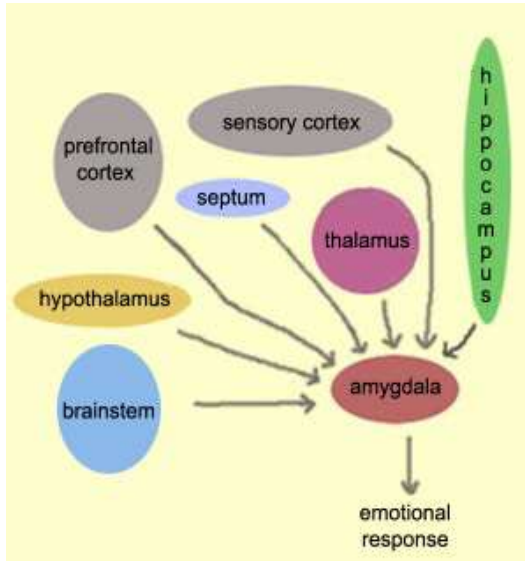

Figure 2. The hippocampus and amygdala are responsible for creating a full memory event and connecting an emotional response to it.

(McGill University, 2018).

In regard to particular brain structures, Levitin explains that some structures have known functions. In memory systems, the hippocampus and areas related to it are responsible for forming new memories (Levitin, 2007, p. 166). The amygdala, next to the hippocampus, is the center for emotion and has more recently been shown to be involved with memory, too (2007). It is highly activated by an experience or memory that has strong emotion connected to it (Levitin, 2007, p. 166).

The McGill website adds that the amygdala receives information from the hippocampus and many other structures, all of which are connected and deeply involved with explicit memory (McGill University, 2018). The hippocampus takes all of the sensory input from a situation (what one heard, saw, smelled, and the emotions one felt) and blends them together to form a single memory. It is believed that the hippocampus is the reason one can "play a memory back" in one's mind. Spatial memory seems to be confined to the right hippocampus, which is thought to create a mental map of space (2018).

Montinaro (2010) adds that the activity of the right hippocampal area is correlated with the percentage rate of success in recalling a musical passage, demonstrating that this area plays an important role in musical memory (Montinaro, 2010, p. 75). The hippocampus, the temporal lobes, and some of the structures connected to them are essential for the consolidation of long-term memory (McGill University, 2018). (See the body of this paper for a full explanation of memory consolidation.)

The cerebellum and basal ganglia (not pictured) continually process rhythm and meter. The cerebellum helps to track a beat and is engaged when we hear music that is familiar or music we like (Levitin, 2007, p. 174). Music processing involves nearly every region of the brain and all of its neural subsystems (Levitin, 2007 p. 85). Music listening, performing, and composing engage regions throughout the brain, bilaterally (Peretz \& Zatorre, 2005). Performing requires the frontal lobes for planning, and the motor cortex and the sensory cortex for tactile feedback. Reading music requires the visual cortex in the occipital lobe in the back of the head (Levitin, 2007, p. 86). 
On processing, Levitin (2007) explains that the left hemisphere areas are needed to process musical structure. For example, the left hemisphere processes whether chords occur where they normally belong or not. Attention to music syntax and structure shows activation in the left frontal cortex, in a subsection which also processes violations to what we expect to hear (Levitin, 2007, p. 129). Beyond this, music structure processing requires both hemispheres (Levitin, 2007, p. 129). For example, the right hemisphere has been found to process pitch contour (Peretz, 1992, as cited in Levitin, 2007, p. 173).

Overall, the brain receives a great deal of information that it may process in parallel and finds a way to bind it together to produce an understandable, audible event (Levitin, 2007, p. 88). "Our perceptions are the end product of a long chain of neural events that give us the illusion of an instantaneous image" (Levitin, 2007, p 98). (See the Human Memory Systems section of this paper.)

Other research in music presents strong evidence and new modeling for how music processing operates in the brain. However, Zatorre \& Zatare (2012) remind us that current research may highlight "our lack of knowledge, or our imprecise models, [more] than it demonstrates solid understanding of the neural mechanisms underlying musically relevant behaviors. We are far from achieving a coherent understanding of all of the subprocesses that lead to the seemingly effortless recognition of a simple tune" (Zatorre \& Zarate, 2012, p. 286).

\section{Brain Operation for Procedural (Motor) Skills}

The McGill University website describes three portions of how a voluntary motor movement happens. First the objective of a movement is defined and its broad necessary steps are planned in the pre-frontal cortex. The frontal cortex gets information from the parietal cortex for spatial perception, so that it may analyze where one's body parts currently are. The basal ganglia participate in this, too (McGill University, 2018).

Continuing, the motor cortex includes two areas just rostral to it, the premotor cortex (PMC) and the supplementary motor area (SMA). These are the secondary motor areas. In the second step, the PMA (the pre-motor area) and the SMA work with the cerebellum to specify the precise contractions of the various muscles that will be required, and to specify the coordinates or angles in space that are involved. This is how variables are sent to a generalized motor program (McGill University, 2018). (See the Auditory Motor Learning section of this paper for more detail on generalized motor programs.) 
Finally, the primary motor cortex, the brainstem, and the spinal cord produce the contractions of all the muscles needed for the chosen movement. The primary motor cortex determines how much force each muscle group must exert and then sends this information to the spinal motor neurons that generate the movement itself, as well as the postural adjustments needed (McGill University, 2018).

Activity in the premotor areas, the PMA and SMA, is often analyzed in music research, because the premotor cortex (PMC) is a crucial supplementary structure for auditory processing. The PMC is divided into two parts, the ventral PMC (VPMC) and the dorsal PMC (dPMC). They are both important nodes, critical arrival points, for particular kinds of processing in the auditory motor network (McGill University, 2018).

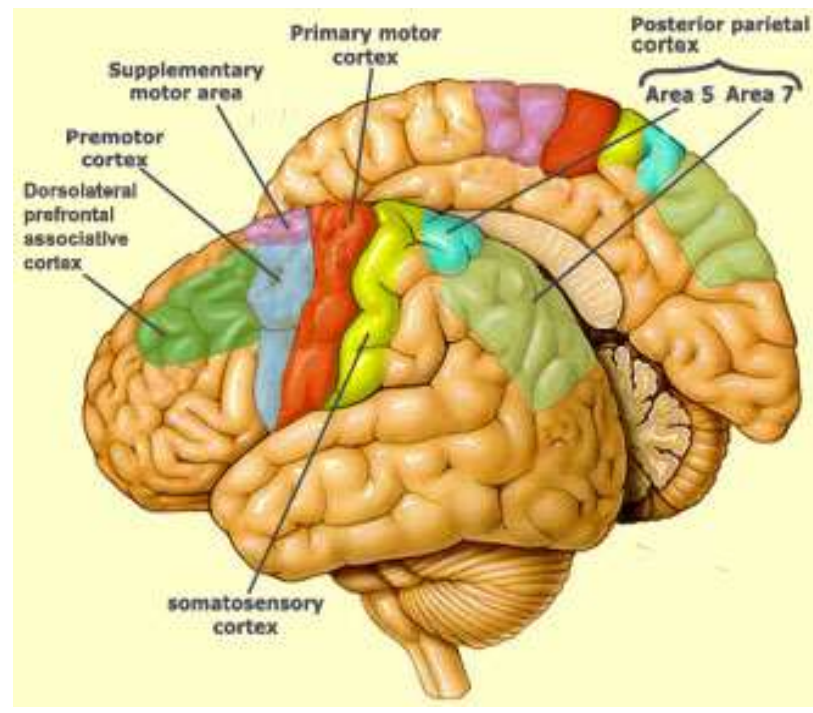

Figure 3. Sensory cortices and some important supplemental brain areas.

(McGill University, 2018).

\section{The Dorsal and Ventral Streams in Auditory Processing}

Zatorre \& Zarate (2012) state that "distinct patterns of anatomical connections within auditory cortex suggest that there are at least two auditory processing streams stemming from primary auditory cortex, each of which may contribute to processing different higher-order aspects of auditory stimuli" (Zatorre \& Zarate, 2012, p. 273). Anterior parts of the primary auditory cortex and posterior parts of the auditory cortex send different information outward to other associated brain areas for processing (2012). This is a dual-stream model for auditory perception.

Researchers have suggested specific models for how these two streams operate. Zatorre \& Zarate (2012) claim that there is "evidence that higher-order processing of musical stimuli . . engages 
regions within the dorsal (upper) auditory stream" (Zatorre \& Zarate, 2012, p. 273). Currently, it is believed that the dorsal stream, in the $\mathrm{APMC}$, carries spatial information and is related to taking an action (Zatorre \& Zarate, 2012, p. 275).

A study by Baumann et al (2007) concerning audio-motor networks in pianists and nonmusicians explains that the dorsal auditory stream guides auditory motor actions, by recognizing patterns of how things are happening in the music and making decisions about producing a motor motion. The dorsal auditory stream is known as the "how-do" portion of a dual-stream model of auditory processing (Baumann et al, p. 72). For this reason, the dorsal stream, in the dPMC, is often involved in music cognition studies.

Baumann et al. (2007) explain that the dPMC (dorsal Pre-Motor Cortex) is engaged when people learn, play, or imagine music. In musicians, it is engaged when listening without playing, when playing without being able to hear oneself, and when hearing or learning to play new melodies. The dPMC is involved with abstract and higher-level aspects of sensorimotor integration (Baumann et al, 2007).

The auditory ventral (lower) stream, in the VPMC, (ventral Pre-Motor Cortex) is thought to process the meaning of sound, concepts, identification of events, and any information that tells the brain "what" it has heard (Baumann et al, 2007, p. 72). The auditory ventral stream is the "what" portion of what was heard (Baumann, 2007).

Secondary sensorimotor areas such as the $\mathrm{dPMC}$, pre-SMA, the SMA, inferior parietal lobule (IPL), and motor-related areas such as the basal ganglia and cerebellum, are activated together as part of an audio-motor network (Baumann, 2007).

For voluntary motor motions, a number of areas were significantly activated, namely, bilateral pre-SMA, SMA, VPMC, IFG (pars opercularis) and basal ganglia. The SMA seemed to be more active when motions were top-down, voluntary motions. The $\mathrm{dPMC}$ was found to activate specifically for voluntary motion. However, the cerebellum was constantly activated in one hemisphere or the other whether motion was voluntary or not. 
Here is a brief explanation of some of the supplementary structures of the brain that are implicated in music cognition research in this paper.

pre-SMA pre-Supplementary Motor Area; works together with the Supplementary Motor Area to prepare motions and to communicate with the primary motor cortex (Wollman et al., 2018).

STG the Superior temporal gyrus; a site of multi-sensory integration, it associates auditory information (Herholz et al., 2012).

STP the Superior temporal gyrus; acts as an auditory-motor interface (Brown \& Penhune, 2018).

STS the Superior temporal sulcus; used in higher-level auditory processing like melody recognition (Brown \& Penhune, 2018).

SPC the Superior parietal cortex; involved with spatial orientation and attention, receives sensory input from the hands (Brown \& Penhune, 2018).

IPC the Inferior parietal cortex; linked to the ability to direct movements toward locations in space (Mattingley et al., 1998; Gnadt \& Andersen, 1988, as cited in Brown \& Penhune, 2018, p. 1675).

IPS the Intra-parietal sulcus; capable of mental manipulations of musical material (Zatorre, 2012).

PP the Planum polare; related to the $\mathrm{VPMC}$, the auditory ventral stream via the ventral PreMotorCortex (Baumann, 2007).

PT

the Planum temporale; related to the $\mathrm{dPMC}$, the auditory dorsal stream via the dorsal PreMotor Cortex (Baumann, 2007). 


\section{References}

Akiva-Kabiri, L., Vecchi, T., Granot, R., Basso, D., \& Schön, D. (2009). Memory for tonal pitches. Annals of the New York Academy of Sciences 1169, 266-69. doi:10.1111/j.1749-6632.2009.04787.x.

Allen, E. J., Burton, P. C., Mesik, J., Olman, C. A., \& Oxenham, A. J. (2019). Cortical correlates of attention to auditory features. Journal of Neuroscience 39(17), 3292-3300. doi:10.1523/JNEUROSCI.058818.2019.

Allen, S. E. (2012). Memory stabilization and enhancement following music practice. Psychology of Music 41(6), 794-803. doi:10.1177/0305735612463947.

Allen, S. E., \& Duke, R.A. (2013). The Effects of limited, restricted music practice on overnight memory enhancement. UPDATE: Applications of Research in Music Education 32(1), 67-73. doi:10.1177/8755123313502350.

American Psychological Association. (2020). APA Dictionary of psychology. https://dictionary.apa.org/transfer-of-training.

Antony, J. W, Gobel, E. W., O'Hare, J. K., Reber, P. J., \& Paller, K. A. (2012). Cued memory reactivation during sleep influences skill learning. Nature Neuroscience 15(8), 1114-16. doi:10.1038/nn.3152.

Bailes, F. (2009). Psychology for musicians: Understanding and acquiring the skills. British Journal of Music Education 26(2), 227-30. doi:10.1017/\$0265051709008468.

Baumann, S., Koeneke, S., Schmidt, C. F., Meyer, M., Lutz, K., \& Jancke, L. (2007). A Network for audiomotor coordination in skilled pianists and non-musicians. Brain Research 1161, 65-78. doi:10.1016/j.brainres.2007.05.045.

Bianco, R., Novembre, G., Keller, P. E., Scharf, F., Friederici, A. D., Villringer, A., \& Sammler, D. (2016). Syntax in action has priority over movement selection in piano playing: An ERP study. Journal of Cognitive Neuroscience 28(1), 41-54. doi:10.1162/jocn_a_00873.

Bjork, E. L., \& Bjork., R. A. (Eds) (1996). Memory. Academic Press.

Bjork, R. A. (2018). Being suspicious of the sense of ease and undeterred by the sense of difficulty: Looking back at Schmidt and Bjork (1992). Perspectives on Psychological Science 13(2), 146-48. doi:10.1177/1745691617690642.

Bolhuis, J. J., \& Moorman, S. (2015). Birdsong memory and the brain: In search of the template. Neuroscience and Biobehavioral Reviews, 50, 41-55. https://doiorg.wvu.idm.oclc.org/10.1016/j.neubiorev.2014.11.019.

Bradley, M. M. \& Keil, A. (2021). Science Direct: Event-related potential. https://www.sciencedirect.com/topics/medicine-and-dentistry/event-related-potential. 
Brain Injury E-Learning Resource. (2017). Introduction to the brain: Structure of the brain. https://www.acquiredbraininjury-education.scot.nhs.uk/introduction-to-the-brain/structure-ofthe-brain/lobes-of-the-brain/.

Brown, R., \& Palmer, C. (2012). Auditory-motor learning Influences auditory memory for music. Memory and Cognition 40(4), 567-78. doi:10.3758/s13421-011-0177-x.

Brown, R. M., \& Penhune, V. B. (2018). Efficacy of auditory versus motor learning for skilled and novice performers. Journal of Cognitive Neuroscience 30(11), 1657-82. doi:10.1162/jocn_a_01309.

Caramiaux, B., Bevilacqua, F., Wanderley, M. M., \& Palmer, C. (2018). Dissociable effects of practice variability on learning motor and timing skills. PLOS ONE 13(3), 1-18.

doi:10.1371/journal.pone.0193580.

Cash, C. D. (2009). Effects of early and late rest intervals on performance and overnight consolidation of a keyboard sequence. Journal of Research in Music Education 57(3), 252-66. doi:10.1177/0022429409343470.

Cash, C. D., Allen, S. E., Simmons, A. L., \& Duke, R. A. (2014). Effects of model performances on music skill acquisition and overnight memory consolidation. Journal of Research in Music Education 62(1), 89-99. doi:10.1177/0022429413520409.

Chaffin, R., Demos, A. \& Logan, T. (2016). Performing from memory. In Oxford Handbook of Music Psychology ( $2^{\text {nd }}$ ed). Hallam, S., Cross, I. and Thaut, M. (Eds). (pp. 559-571). Oxford University Press.

Cohen, A. J. (2016). From auditory focus, to auditory influence, to multimodal experience in psychomusicological research. Psychomusicology: Music, Mind and Brain, 26(2), 99-100. https://doi-org.wvu.idm.oclc.org/10.1037/pmu0000149.

Colley, I. D., Keller, P. E., \& Halpern, A. R. (2018). Working memory and auditory imagery predict sensorimotor synchronization with expressively timed music. Quarterly Journal of Experimental Psychology 71(8), 1781-96. doi:10.1080/17470218.2017.1366531.

Davidson-Kelly, K., Moran, N., Schaefer, R. S., \& Overy, K. (2015). Total inner memory: Deliberate uses of multimodal musical imagery during performance preparation. Psychomusicology: Music, Mind and Brain 25(1), 83-92. doi:10.1037/pmu0000091.

Debarnot, U., Castellani, E., Guillot, A., Giannotti, V., Dimarco, M., \& Sebastiani, L. (2012). Declarative interference affects off-line processing of motor imagery learning during both sleep and wakefulness. Neurobiology of Learning and Memory 98(4), 361-67. doi:10.1016/j.nlm.2012.10.009.

Demos, A. P., (2013). The Perception of movement through musical sound: Towards a dynamical systems theory of music performance. PhD diss. https://opencommons.uconn.edu/dissertations/155. 
Deutsch, D. (Ed). (1999). The Psychology of music. (2 ${ }^{\text {nd }}$ ed). Academic Press.

Ding, Y., Gray, K., Forrence, A., Wang, X., \& Huang, J. (2018). A Behavioral study on tonal working memory in musicians and non-musicians. PLOS ONE 13(8), 1-18. doi:10.1371/journal.pone.0201765.

Dowling, W. J., \& Tillman, B. (2014). Memory improvement while hearing music: Effects of structural continuity on feature binding. Music Perception 32(1), 11-32. doi:10.1525/MP.2014.32.1.11.

Duke, R. A., Cash, C. D., \& Allen., S. E. (2011). Focus of attention affects performance of motor skills in music. Journal of Research in Music Education 59(1), 44-55. doi:10.1177/0022429410396093.

Duke, R. A., Allen, S. E., Cash, C. D., \& Simmons, A. L. (2019). Effects of early break intervals on musicians' and non-musicians' skill learning. Psychology of Music 47, 83-95. doi: 10.1177/0305735617735373.

Duke, R. A., Cash, C. D., \& Simmons, A. L. (2009a). It's not how much, it's how. Characteristics of practice behavior and retention of performance skills. Journal of Research in Music Education 56(4), 310321. doi: $10.1177 / 0022429408328851$.

Duke, R. A., Allen, S. E., Cash, C. D., \& Simmons, A. L. (2009b). Effects of early and late rest breaks during training on overnight memory consolidation of a keyboard melody. Annals of the New York Academy of Sciences 1169, 169-72. doi:10.1111/j.1749-6632.2009.04795.x.

Fine, P. A., Goldemberg, R., Wise, K. J., \& Bravo, A. (2015). Performing musicians' understanding of the terms 'mental practice' and 'score analysis.' Psychomusicology: Music, Mind and Brain 25(1), 69-82. doi:10.1037/pmu0000068.

Finney, S. A., \& Palmer, C. (2003). Auditory feedback and memory for music performance: Sound evidence for an encoding effect. Memory and Cognition 31(1), 51. doi:10.3758/BF03196082.

Freedberg, M., Schacherer, J., \& Hazeltine, E. (2015). Incidental learning of rewarded associations bolsters learning on an associative task. Journal of Experimental Psychology: Learning, Memory, and Cognition 42(5), 786-803. doi:10.1037/xlm0000201.

Furuya, S., Furukawa, Y., Uehara, K., \& Oku, T. (2018). Probing sensorimotor integration during musical performance. Annals of the New York Academy of Sciences 1423(1), 211-18. doi:10.1111/nyas.13619.

Gazzaley, A., \& Nobre, A. C. (2012). Top-down modulation: Bridging selective attention and working memory. Trends in Cognitive Sciences 16(2), 129-35.

Gingras, B., Pearce, M. T., Goodchild, M., Dean, R. T., Wiggins, G., \& McAdams, S. (2016). Linking melodic expectation to expressive performance timing and perceived musical tension. Journal of Experimental Psychology. Human Perception and Performance, 42(4), 594-609. https://doiorg.wvu.idm.oclc.org/10.1037/xhp0000141. 
Godøy, R. I., \& Leman, M. (2009). Musical gestures: Sound, movement, and meaning. New York, NY: Routledge.

Gordon, E. E. (2012). Learning sequences in music: A Contemporary music learning theory. (2012 ed). GIA Publishing.

Green, B. (1986). The Inner game of music. Doubleday.

Grimault, S., Nolden, S., Lefebvre, C., Vachon, F., Hyde, K., Peretz, I., Zatorre, R., Robitaille, N., \& Jolicoeur, P. (2014). Brain activity Is related to individual differences in the number of items stored in auditory short-term memory for pitch: Evidence from magnetoencephalography. Neurolmage 94, 96-106. doi:10.1016/j.neuroimage.2014.03.020.

Groussard, M., Viader, F., Landeau, B., Desgranges, B., Eustache, F., \& Platel, H. (2009). Neural correlates underlying musical semantic memory. Annals of the New York Academy of Sciences 1169, 27881. doi:10.1111/j.1749-6632.2009.04784.x.

Halpern, A. R. (2012). Dynamic aspects of musical imagery. Annals of the New York Academy of Sciences 1252(1), 200-205. doi:10.1111/j.1749-6632.2011.06442.x.

Hamel, R., Côté, K., Matte, A., Lepage, J. F., \& Bernier, P. M. (2019). Rewards interact with repetitiondependent learning to enhance long-term retention of motor memories. Annals of the New York Academy of Sciences 1452(1), 34-51. doi:10.1111/nyas.14171.

Hansen, P., \& Oxoby, R. J. (2017). An Earned presence: Studying the effect of multi-task improvisation systems on cognitive and learning capacity. Connection Science 29(1), 77-93.

doi:10.1080/09540091.2016.1277692.

Heck, D. H., Kozma, R., and Kay, L. M. (2019). The Rhythm of memory: How breathing shapes memory function. Journal of Neurophysiology 122(2), 563-71. doi:10.1152/jn.00200.2019.

Herholz, S. C., Halpern, A. R., \& Zatorre, R. J. (2012). Neuronal correlates of perception, imagery, and memory for familiar tunes. Journal of Cognitive Neuroscience 24(6), 1382-97.

doi:10.1162/jocn_a_00216.

Herholz, S. C., Lappe, C., Knief, A., \& Pantev, C. (2009). Imagery mismatch negativity in musicians. Annals of the New York Academy of Sciences 1169, 173-77. doi:10.1111/j.17496632.2009.04782.x.

Herholz, S. C., \& Zatorre, R. J. (2012). Musical training as a framework for brain plasticity: Behavior, function, and structure. Neuron 76(3), 486-502. doi:10.1016/j.neuron.2012.10.011.

Hernandez-Ruiz, E. (2019). How Is music processed? Tentative answers from cognitive neuroscience. Nordic Journal of Music Therapy 28(4), 315-32. doi:10.1080/08098131.2019.1587785. 
Highben, Z., \& Palmer, C. (2004). Effects of auditory and motor mental practice in memorized piano performance. Bulletin of the Council for Research in Music Education 159, 58-65. Stable URL: https://www.jstor.org/stable/40319208.

Holmes, P. (2005). Imagination in practice: A Study of the integrated roles of interpretation, imagery and technique in the learning and memorisation processes of two experienced solo performers. British Journal of Music Education 22(3), 217-35. doi:10.1017/S0265051705006613.

Hubbard, T. L. (2010). Auditory imagery: Empirical findings. Psychological Bulletin 136(2), 302-29. doi:10.1037/a0018436.

Jain, S., \& Nataraja, N. P. (2019). The Effect of fatigue on working memory and auditory perceptual abilities in trained musicians. American Journal of Audiology 28(25), 483-94. doi:10.1044/2019_AJA-IND50-18-0102.

Katus, T., \& Eimer, M. (2018). Independent attention mechanisms control the activation of tactile and visual working memory representations. Journal of Cognitive Neuroscience, 30(5), 644-655. https://doi-org.wvu.idm.oclc.org/10.1162/jocn_a_01239.

Keller, P. E. (2012). Mental imagery in music performance: Underlying mechanisms and potential benefits. Annals of the New York Academy of Sciences 1252(1), 206-13. doi:10.1111/j.17496632.2011.06439.x.

Kerr, R., \& Booth, B. (1978). Specific and varied practice of motor skill. Perceptual and Motor Skills 46(2), 395-401. doi:10.1177/003151257804600201.

Korenman, L. M., \& Peynircioglu, Z. F. (2007). Individual differences in learning and remembering music: Auditory versus visual presentation. Journal of Research in Music Education, 55(1), 48-64. https://doi-org.wvu.idm.oclc.org/10.1177/002242940705500105.

Krumansl, C. L. (1990). Cognitive foundations of musical pitch. New York: Oxford University Press.

Kuriyama, K., Stickgold, R., \& Walker, M. P. (2004). Sleep-dependent learning and motor-skill complexity. Learning and Memory, 11(6), 705.

Kurtuldu, M., Güçlü, H. (2010). Piyano eğitiminde planlı ve eşit tekrar çalışmasının öğrencilerin başarısı üzerindeki etkileri. Elektronik Sosyal Bilimler Dergisi 9, 196-204.

Kussner, M., Eerola, T., \& Fujioka, T. (2019). Music, emotion, and visual imagery: Where are we now? Psychomusicology: Music, Mind and Brain 29 (2-3), 59-61. doi:10.1037/pmu0000245.

Leaver, A. M., Van Lare, J., Zielinski, B., Halpern, A. R., \& Rauschecker, J. P. (2008). Brain activation during anticipation of sound sequences. Journal of Neuroscience, 2477-85. doi:10.1523/JNEUROSCI.4921-08.2009.

Leman, M. (2007). Embodied music cognition and mediation technology. Cambridge, MA: MIT Press. 
Leman M., \& Maes, P. J. (2014). The Role of embodiment in the perception of music. Empirical Musicology Review 9(3-4), 236-246.

Leman M., Maes P. J., Nijs, L., \& Van Dyck, E. (2018). What Is embodied music cognition? In: Bader, R. (ed) Springer Handbook of Systematic Musicology. Springer Handbooks. Springer, Berlin, Heidelberg. https://doi.org/10.1007/978-3-662-55004-5_34.

Levitin, D. J. (2007) This is your brain on music. Plume.

Levitin, D. J. (2011). Foundations of cognitive psychology: Core readings ( $2^{\text {nd }}$ ed). Pearson Education, Inc.

Levitin, D. J., \& Tirovolas, A. K. (2009). Current advances in the cognitive neuroscience of music. Annals of the New York Academy of Sciences 1156, 211-31. doi:10.1111/j.1749-6632.2009.04417.x.

Lisboa, T., Demos, A. P., \& Chaffin, R. (2018). Training thought and action for virtuoso performance. Musicae Scientiae 22(4), 519-38. doi:10.1177/1029864918782350.

Loimusalo, N. J., and Huovinen, E. (2018). Memorizing silently to perform tonal and nontonal notated music: A Mixed-methods study with pianists. Psychomusicology: Music, Mind and Brain 28(4), 222-39. doi:10.1037/pmu0000227.

Lorenzo, A. G., Ruiz, R. O., Gea, E. V., \& Ortiz, O. G. (2015). Validación de dos instrumentos de medida para evaluar las creencias motivacionales y las estrategias de práctica en los procesos de memorización en la interpretación pianística. (A. Simpson, Trans.) Revista Electrónica de LEEME 36, 31-48.

http://search.ebscohost.com.www.libproxy.wvu.edu/login.aspx?direct=true\&db=a9h\&AN=1165 02006.

McDevitt, E. A., Sattari, N., Duggan, K. A., Cellini, N., Whitehurst, L. N., Perera, C., Reihanabad, N., Granados, S., Hernandez, L., \& Mednick, S. C. (2018). The impact of frequent napping and nap practice on sleep-dependent memory in humans. Scientific Reports, 8(1), 1. https://doiorg.wvu.idm.oclc.org/10.1038/s41598-018-33209-0.

McGill University. (2018). The Brain from Top to Bottom: The Motor cortex. https://thebrain.mcgill.ca/flash/a/a_06/a_06_cr/a_06_cr_mou/a_06_cr_mou.html.

McGill University. (2018). The Brain from Top to Bottom: The Amygdala and its allies. https://thebrain.mcgill.ca/flash/a/a_04/a_04_cr/a_04_cr_peu/a_04_cr_peu.html.

Maes, P. J., \& Leman, M. (2013). The Influence of body movements on children's perception of music with an ambiguous expressive character. PLoS-One 8(1), E54682.

Maes, P. J.,Leman, M., Palmer, C., \& Wanderley, M. (2014). Action-based effects on music perception. Frontiers in Psychology. 4(1008), 1-14.

Maes, P. J., Wanderley, M., and Palmer, C. (2015). The Role of working memory in the temporal control of discrete and continuous movements. Experimental Brain Research 233(1), 263-73. doi:10.1007/s00221-014-4108-5. 
Mathias, B., Gehring, W. J., \& Palmer, C. (2016). Auditory N1 reveals planning and monitoring processes during music performance. Psychophysiology 54(2), 235-47. doi:10.1111/psyp.12781.

Mathias, B., Gehring, W. J., \& Palmer, C. (2019). Electrical brain responses reveal sequential constraints on planning during music performance. Brain Sciences 9(2), 25. doi:10.3390/brainsci9020025.

Mathias, B., Tillmann, B., \& Palmer, C. (2016). Sensory, cognitive, and sensorimotor learning effects in recognition memory for music. Journal of Cognitive Neuroscience 28(8), 1111-26. doi:10.1162/jocn_a_00958.

Mednick, S., Nakayama, K., \& Stickgold, R. (2003). Sleep-dependent learning: a nap is as good as a night. Nature Neuroscience, 6(7), 697. https://doi-org.wvu.idm.oclc.org/10.1038/nn1078.

Meister, I. G., Krings, T., Foltys, H., Boroojerdi, B., Müller, M., Töpper, R., \& Thron, A. (2004). Playing piano in the mind-an FMRI study on music imagery and performance in pianists. Cognitive Brain Research 19(3), 219. doi:10.1016/j.cogbrainres.2003.12.005.

Meyer, R. K., \& Palmer, C. (2003). Temporal and motor transfer in music performance. Music Perception, 21(1), 81-104. https://doi-org.wvu.idm.oclc.org/10.1525/mp.2003.21.1.81.

Middlebrooks, C. D., Kerr, T., \& Castel, A. D. (2017). Selectively distracted: Divided attention and memory for important information. Psychological Science 28(8), 1103-15.

doi:10.1177/0956797617702502.

Mishra, J. (2016). Playing from memory. American Music Teacher 65(6), 12-16. http://search.ebscohost.com.www.libproxy.wvu.edu/login. aspx?direct=true\&db=a9h\&AN=1154 70064.

Moldwin, T., Schwartz, O., \& Sussman, E. S. (2017). Statistical learning of melodic patterns influences the brain's response to wrong notes. Journal of Cognitive Neuroscience 29(12), 2114-22. doi:10.1162/jocn_a_01181.

Montinaro, A. (2010). The Musical brain: Myth and science. World Neurosurgery 73(5), 442-53. doi:10.1016/j.wneu.2010.02.060.

Moorman, S., Mello, C. V., \& Bolhuis, J. J. (2011). From songs to synapses: Molecular mechanisms of birdsong memory. BioEssays, 33(5), 377-385. https://doiorg.wvu.idm.oclc.org/10.1002/bies.201000150.

Münte, T. F., Altenmüller, E., \& Jäncke, L. (2002). Opinion: The Musician's brain as a model of neuroplasticity. Nature Reviews Neuroscience 3(6), 473-78. doi:10.1038/nrn843.

Myers, N. E., Stokes, M. G., \& Nobre, A. C. (2017). Prioritizing information during working memory: Beyond sustained internal attention. Trends in Cognitive Sciences 21(6), 449-61. doi:10.1016/j.tics.2017.03.010. 
Nakamura, N. H., Fukunaga, M., \& Oku Y. (2018). Respiratory modulation of cognitive performance during the retrieval process. PLOS ONE 13(9), 1-17. doi:10.1371/journal.pone.0204021.

National Autonomous University of Mexico (UNAM), Institute of Cellular Physiology. (2013). A Brief introduction to the brain: Nervous system. http://www.ifc.unam.mx/Brain/cercox.htm.

The National Center for Biotechnology Information. (2001). Bookshelf: The Auditory cortex. https://www.ncbi.nlm.nih.gov/books/NBK10900/.

Nichols, B. E., Wöllner, C., \& Halpern, A. R. (2018). Score one for jazz: Working memory in jazz and classical musicians. Psychomusicology: Music, Mind, and Brain 28(2), 101-7. doi:10.1037/pmu0000211.

Oura, Y. (1991). Constructing a representation of a melody: Transforming melodic segments into reduced pitch patterns operated on by modifiers. Music Perception, 9, 251-266.

Oura, Y., \& Hatano, G. (2004). Parsing and memorizing tonal and modal melodies. Japanese Psychological Research 46(4), 308-21. doi:10.1111/j.1468-5584.2004.00263.x.

Oxenham, A. J. (2018). How we hear: The Perception and neural coding of sound. Annual Review of Psychology 69, 27-50. doi:10.1146/annurev-psych-122216-011635.

Pacheco, U., Pilar, A., and Parmentier, F. B. R. (2016). Happiness increases distraction by auditory deviant stimuli. British Journal of Psychology 107(3), 419-33. doi:10.1111/bjop.12148.

Palmer, C. (1989). Mapping musical thought to musical performance. Journal of Experimental Psychology: Human Perception and Performance 15(2), 331-46. doi:10.1037/00961523.15.2.331.

Palmer, C. (1997). Music performance. Annual Review of Psychology 48(1), 115. doi:10.1146/annurev.psych.48.1.115.

Palmer, C. (2015). Listening, imagining, performing: Melody as a life cycle of musical thought. Music Perception 33(1), 3-11. doi:10.1525/mp.2015.33.1.3.

Palmer, C., \& Drake, C. (1997). Monitoring and planning capacities in the acquisition of music performance skills. Canadian Journal of Experimental Psychology 51(4), 369. doi:10.1037/11961961.51.4.369.

Palmer, C., \& van de Sande, C. (1993). Units of knowledge in music performance. Journal of Experimental Psychology: Learning, Memory, and Cognition 19(2), 457-70. doi:10.1037/0278-7393.19.2.457.

Palmer, C., \& van de Sande, C. (1995). Range of planning in music performance. Journal of Experimental Psychology. Human Perception and Performance 21(5), 947. doi:10.1037/0096-1523.21.5.947. 
Palmer, C., Mathias, B., \& Anderson, M. (2012). Sensorimotor mechanisms in music performance: Actions that go partially wrong. Annals of the New York Academy of Sciences 1252(1), 185-91. doi:10.1111/j.1749-6632.2011.06427.x.

Palmer, C., \& Meyer, R. K. (2000). Conceptual and motor learning in music performance. Psychological Science 11(1), 63. doi:10.1111/1467-9280.00216.

Pearce, M. T., and Christensen, J. F. (2012). Conference report: The Neurosciences and Music-IV-Learning and Memory. Psychomusicology: Music, Mind and Brain 22(1), 70-73. doi:10.1037/a0027235.

Peretz, I. (1992). The role of contour and intervals in the recognition of melody parts: Evidence from cerebral asymmetries in musicians. Neuropsychologia 30(3), 277. doi: 10.1016/00283932(92)90005-7.

Peretz, I. (2002). Brain specialization for music. Neuroscientist, 8(4), 372. https://doi-org.wvu.idm.oclc.org/10.1177/107385840200800412.

Peretz, I., Gosselin, N., Belin, P., Zatorre, R. J., Plailly, J., \& Tillmann, B. (2009). Music lexical networks. Annals of the New York Academy of Sciences 1169, 256-65. doi:10.1111/j.17496632.2009.04557.x.

Peretz, I., \& Zatorre, R. J. (2005). Brain organization for music processing. Annual Review of Psychology, 56(1), 89-114. https://doiorg.www.libproxy.wvu.edu/10.1146/annurev.psych.56.091103.070225.

Pfordresher, P. Q., Palmer, C., and Jungers, M. K. (2007). Speed, accuracy, and serial order in sequence production. Cognitive Science 31(1), 63-98. doi:10.1207/s15516709cog3101_3.

Rosenkranz, K., Williamon, A., Rothwell, J. C. (2007). Motorcortical excitability and synaptic plasticity is enhanced in professional musicians. Journal of Neuroscience 27(19), 5200-5206. doi: 10.1523/JNEUROSCI.0836-07.2007.

Saintilan, N. (2015). The Use of imagery during the performance of memorized music. Psychomusicology: Music, Mind and Brain 24(4), 309-15. doi:10.1037/pmu0000080.

Sanders, L. (2012). Practice piano while you sleep. Science News 182(2), 10. doi:10.1002/scin.5591820209.

Schmidt, R. A. (1975). A Schema theory of discrete motor skill learning. Psychological Review 82(4), 22560. doi:10.1037/h0076770.

Schmidt, R. A., \& Bjork, R. A. (1992). New conceptualizations of practice: Common principles in three paradigms suggest new concepts for training. Psychological Science 3(4), 207-17. doi:10.1111/j.1467-9280.1992.tb00029.x. 
Schulkind, M. D. (2009). Is Memory for Music Special? Annals of the New York Academy of Sciences 1169, 216-24. doi:10.1111/j.1749-6632.2009.04546.x.

Schulze, K., \& Koelsch, S. (2012). Working memory for speech and music. Annals of the New York Academy of Sciences, 1252(1), 229-236. https://doi-org.wvu.idm.oclc.org/10.1111/j.17496632.2012.06447.x

Silva, S., Dias, C., \& Castro, S. L. (2019). Domain-specific expectations in music segmentation. Brain Sciences 9(169), 1-20. doi:10.3390/brainsci9070169.

Simmons, A. L. (2007). Effects of practice variability and distribution of practice on musicians' performance of a procedural skill. PhD diss. The University of Texas at Austin.

Simmons, A. L. (2012). Distributed practice and procedural memory consolidation in musicians' skill learning. Journal of Research in Music Education 59(4), 357-68.

doi:10.1177/0022429411424798.

Simmons, A. L., \& Duke, R. A. (2006). Effects of sleep on performance of a keyboard melody. Journal of Research in Music Education 54(3), 257-69. doi:10.2307/4151346.

Soderstrom, N. C., \& Bjork, R. A. (2015). Learning versus performance: An Integrative review. Perspectives on Psychological Science 10(2), 176-99. doi:10.1177/1745691615569000.

Smit, E. A., \& Sadakata, M. (2018). The Effect of handedness on spatial and motor representation of pitch patterns in pianists. PLOS ONE 13(5), 1-14. doi:10.1371/journal.pone.0195831.

Snyder, B. (2000). Music and memory: An Introduction. Cambridge: MIT Press.

Stalinski, S. M., and Schellenberg, E. G. (2013). Listeners remember music they like. Journal of Experimental Psychology. Learning, Memory and Cognition 39(3), 700-716. doi:10.1037/a0029671.

Talamini, F., Altoè, G., Carretti, B., \& Grassi, M. (2017). Musicians have better memory than nonmusicians: A Meta-analysis. PLoS ONE, 12(10), 1-21. https://doiorg.wvu.idm.oclc.org/10.1371/journal.pone.0186773.

The Gordon Institute for Music Learning. (2021). Specific applications to music instruction. https://giml.org/mlt/applications/\#instrumental.

University of Calgary. (2021). School of Creative and Performing Arts. https://arts.ucalgary.ca/creativeperforming-arts.

Van Dyck, E., Vansteenkiste, P., Lenoir, M., Lesaffre, M., \& Leman, M. (2014). Recognizing induced emotions of happiness and sadness from dance movement. PLoS-One 9(2), E89773.

Van Hedger, S. C., Hogstrom, A., Palmer, C., \& Nusbaum, H. C. (2015). Sleep consolidation of musical competence. Music Perception 33(2), 163-78. doi:10.1525/MP.2015.33.2.163. 
Walker, M. P. (2005). A Refined model of sleep and the time course of memory formation. Behavioral and Brain Sciences 28(1), 51-104. doi:10.1017/S0140525X05000026.

Webb, T. W., Kean, H. H., \& Graziano, M. S. A. (2016). Effects of awareness on the control of attention. Journal of Cognitive Neuroscience 28(6), 842-51. doi:10.1162/jocn_a_00931.

Wollman, I., Penhune, V., Segado, M., Carpentier, T., \& Zatorre, R. J. (2018). Neural network retuning and neural predictors of learning success associated with cello training. Proceedings of the National Academy of Sciences of the United States of America 115(26) E6056-64. doi:10.1073/pnas.1721414115.

Wöllner, C., \& Halpern, A. R. (2016). Attentional flexibility and memory capacity in conductors and pianists. Attention, Perception and Psychophysics 78(1), 198-208. doi:10.3758/s13414-0150989-z.

Wu, R., Shimi, A., Solis, M., \& Scerif, G. (2018). Learning what to attend to: From the lab to the classroom. Journal of Cognitive Neuroscience 30(12), 1749-56. doi:10.1162/jocn_a_01316.

Yu, K., Wood, W. E., and Theunissen, F. E. (2020). High-capacity auditory memory for vocal communication in a social songbird. Science Advances, 6(46), 1-8. https://doiorg.wvu.idm.oclc.org/10.1126/sciadv.abe0440.

Zajonc, R. B. (2001). Mere exposure: A Gateway to the subliminal. Current Directions in Psychological Science 10(6), 224. doi:10.1111/1467-8721.00154.

Zatorre, R. J. (2012). Beyond auditory cortex: Working with musical thoughts. Annals of the New York Academy of Sciences 1252(1), 222-28. doi:10.1111/j.1749-6632.2011.06437.x.

Zatorre, R. J. (2013). Predispositions and plasticity in music and speech learning: Neural correlates and implications. Science 342(6158), 585-89. doi:10.1126/science.1238414.

Zatorre, R. J., \& Halpern, A. R. (2005). Mental concerts: Musical imagery and auditory cortex. Neuron 47(1), 9-12. doi:10.1016/j.neuron.2005.06.013.

Zatorre, R. \& Zarate, J. (2012). The Human auditory cortex. In Springer Handbook of Auditory Research. (pp. 261-294). Springer Science and Business Media, LLC. Doi:10.1007/978-1-4614-2314-0_10.

Zelano, C., Jiang, H., Zhou, G., Arora, N., Schuele, S., Gottfried, J. A., \& Rosenow, J. (2016). Nasal respiration entrains human limbic oscillations and modulates cognitive function. Journal of Neuroscience 36(49), 12448-67. doi:10.1523/JNEUROSCI.2586-16.2016. 